\title{
Molecular Biomarkers for the Evaluation of Colorectal Cancer
}

\author{
Guideline From the American Society for Clinical Pathology, College of American \\ Pathologists, Association for Molecular Pathology, and American Society of \\ Clinical Oncology
}

\author{
Antonia R. Sepulveda, MD, PhD, ${ }^{1}$ Stanley R. Hamilton, MD, ${ }^{2}$ Carmen J. Allegra, MD, ${ }^{5}$ Wayne Grody, MD, PhD, ${ }^{6}$ \\ Allison M. Cushman-Vokoun, MD, PhD, ${ }^{7}$ William K. Funkhouser, MD, PhD, ${ }^{8}$ Scott E. Kopetz, MD, PhD, ${ }^{3}$ Christopher Lieu, MD, ${ }^{9}$ \\ Noralane M. Lindor, MD, ${ }^{10}$ Bruce D. Minsky, MD, ${ }^{4}$ Federico A. Monzon, MD, ${ }^{11}$ Daniel J. Sargent, PhD, ${ }^{12}$ Veena M. Singh, MD, ${ }^{13}$ \\ Joseph Willis, MD, ${ }^{14}$ Jennifer Clark, SCT, MB(ASCP) ${ }^{\mathrm{cm}},{ }^{15}$ Carol Colasacco, MLIS, ${ }^{16}$ R. Bryan Rumble, MSC, ${ }^{17}$ \\ Robyn Temple-Smolkin, PhD, ${ }^{18}$ Christina B. Ventura, MT(ASCP), ${ }^{16}$ and Jan A. Nowak, MD, PhD ${ }^{19}$
}

Accepted for publication November 11, 2016.

Published as an Early Online Release February 6, 2017.

Supplemental digital content is available for this article at archivhesofpathology.org in the May 2017 table of contents.

From the ${ }^{1}$ Department of Pathology and Cell Biology, Columbia University, New York, NY; Departments of ${ }^{2}$ Pathology, ${ }^{3}$ Gastrointestinal (GI) Medical Oncology, and ${ }^{4}$ Radiation Oncology, University of Texas MD Anderson Cancer Center, Houston; ${ }^{5}$ Division of Hematology and Oncology, University of Florida Medical Center, Gainesville; ${ }^{6}$ Departments of Pathology and Laboratory Medicine, Pediatrics, and Human Genetics, UCLA Medical Center, Los Angeles, CA; ${ }^{7}$ Department of Pathology and Microbiology, University of Nebraska Medical Center, Omaha; ${ }^{8}$ Department of Pathology and Laboratory Medicine, University of North Carolina School of Medicine, Chapel Hill; ${ }^{9}$ Division of Medical Oncology, University of Colorado Denver School of Medicine, Denver; ${ }^{10}$ Department of Medical Genetics, Mayo Clinic, Scottsdale, AZ; ${ }^{11}$ Castle Biosciences, Friendswood, TX; ${ }^{12}$ Department of Health Sciences Research, Mayo Clinic, Rochester, MN; ${ }^{13}$ Biocept, San Diego, CA; ${ }^{14}$ Department of Pathology, Case Western Reserve University, Cleveland, OH; ${ }^{15} \mathrm{ASCP}$ Institute for Science, Technology, and Policy, American Society for Clinical Pathology, Washington, DC; ${ }^{16}$ Laboratory and Pathology Quality Center, College of American Pathologists, Northfield, IL; ${ }^{17}$ American Society of Clinical Oncology, Alexandria, VA; ${ }^{18}$ Association for Molecular Pathology, Bethesda, MD; and ${ }^{19}$ Department of Pathology and Laboratory Medicine, Roswell Park Cancer Institute, Buffalo, NY.

This guideline was developed through collaboration between the American Society for Clinical Pathology, College of American Pathologists, Association for Molecular Pathology, American Society of Clinical Oncology, and American Society for Investigative Pathology and has been jointly published by invitation and consent in the American Journal of Clinical Pathology, Archives of Pathology \& Laboratory Medicine, Journal of Molecular Diagnostics, and Journal of Clinical Oncology. It has been edited in accordance with standards established at the American Journal of Clinical Pathology.

Copyright 2017 American Society for Clinical Pathology, College of American Pathologists, Association for Molecular Pathology, American Society of Clinical Oncology, and American Society for Investigative Pathology.

Corresponding author: Antonia R. Sepulveda, MD, PhD, Department of Pathology \& Cell Biology, Columbia University Medical Center, 630 W 168th Street, VC-14 RM 212, New York, NY 10032; as4400@cumc.columbia.edu.
- Objectives.-To develop evidence-based guideline recommendations through a systematic review of the literature to establish standard molecular biomarker testing of colorectal cancer (CRC) tissues to guide epidermal growth factor receptor (EGFR) therapies and conventional chemotherapy regimens.

Methods.- The American Society for Clinical Pathology, College of American Pathologists, Association for Molecular Pathology, and American Society of Clinical Oncology convened an expert panel to develop an evidence-based guideline to establish standard molecular biomarker testing and guide therapies for patients with CRC. A comprehensive literature search that included more than 4,000 articles was conducted.

Results. - Twenty-one guideline statements were established.

Conclusions. - Evidence supports mutational testing for EGFR signaling pathway genes, since they provide clinically actionable information as negative predictors of benefit to anti-EGFR monoclonal antibody therapies for targeted therapy of CRC. Mutations in several of the biomarkers have clear prognostic value. Laboratory approaches to operationalize CRC molecular testing are presented.

(Arch Pathol Lab Med. 2017;141:625-657; doi: 10.5858/ arpa.2016-0554-CP)

$\mathbf{M}$ olecular testing to select targeted and conventional therapies for patients with colorectal cancer (CRC) has been the focus of a number of recent studies and is becoming standard practice for management of patients with CRC. Molecular markers that predict response to a specific therapy or treatment regimen are known as predictive biomarkers. ${ }^{1}$ Monoclonal antibody therapies that target the epidermal growth factor receptor (EGFR) bind the 
EGFR extracellular domain, blocking EGFR signaling pathways. Anti-EGFR monoclonal antibodies have been the main targeted therapies for $\mathrm{CRC}$ that require knowledge of the mutational status of genes in the pathway as predictive biomarkers of response to these therapies. ${ }^{2-4}$ Initial clinical trial data demonstrated that patients with CRC carrying activating mutations of KRAS affecting exon 2 codons 12 and 13 did not benefit from anti-EGFR monoclonal antibody therapy. ${ }^{2-4}$ Subsequent studies described other mutations in genes of the EGFR signaling pathways involving other exons of KRAS and in NRAS, $B R A F, P I K 3 C A$, and PTEN that may affect response of CRC to anti-EGFR antibody therapies. Guidelines addressing the molecular testing of EGFR pathway genes beyond KRAS have not been established and are needed in clinical practice.

The DNA mismatch repair (MMR) status of CRC may have predictive value in some clinical settings. While testing of CRC for MMR has been recommended for all patients with CRC as a workup test to evaluate for possible Lynch syndrome, ${ }^{5}$ guidelines for the use of MMR as a predictive biomarker of response to therapy have not been reported. Recent molecular biomarker data have shown the importance of microsatellite instability (MSI) testing, a marker of deficient mismatch repair (dMMR), for the selection of patients for immunotherapy (see section on emerging biomarkers below).

Alterations of a number of critical genes in CRC development and progression such as $\mathrm{dMMR}$ and $B R A F$ activating mutations have been shown to affect prognosis, as measured by several metrics of tumor progression or survival. ${ }^{6-8}$ The utility of incorporating prognostic biomarkers in the management of patients with CRC has not been well defined in clinical practice. Defining the utility of information gathered from prognostic molecular biomarkers for clinical management of patients with CRC is warranted.

The postgenome era and the emphasis on precision genomic-based medicine are providing enormous amounts of new data and many promising new molecular cancer biomarkers that may emerge as molecular diagnostic tools that can be used to enhance successful treatment of patients with CRC and other cancers. Laboratories and regulatory agencies are faced with challenges to rapidly and efficiently provide new test results for the management of patients with cancer. Laboratory testing of molecular biomarkers involves the selection of assays, type of specimens to be tested, timing of ordering of tests, and turnaround time for testing results. Recent years have shown that a plethora of technical approaches can effectively be used as long as test specificity and sensitivity meet the clinical needs. While earlier testing approaches were focused on one or a few testing targets, the current need for multiple molecular markers from potentially minute tumor samples is leading to greater use of gene panels such as targeted next-generation sequencing (NGS) cancer panels, which can assay from a few to hundreds of genes and amplicons with known mutational hotspots in cancer.

There is a need for current evidence-based recommendations for the molecular testing of CRC tissues to guide EGFR-targeted therapies and conventional chemotherapy regimens. Therefore, the current recommendations were developed through collaboration of four societies: American Society for Clinical Pathology
(ASCP), College of American Pathologists (CAP), Association for Molecular Pathology (AMP), and American Society of Clinical Oncology (ASCO). This guideline follows well-established methods used in their development as well as for regular updates, such that new advances in the molecular testing for clinical management of CRC can be integrated in future updates of the guideline in a timely manner.

\section{PANEL COMPOSITION}

The ASCP, the CAP Pathology and Laboratory Quality Center (the Center), AMP, and ASCO convened an expert panel consisting of practicing pathologists, oncologists, geneticists, and a biostatistician with expertise and experience in molecular biomarker testing and targeted therapies for CRC. The ASCP, CAP, AMP, and ASCO jointly approved the appointment of the project, cochairs, and expert panel members. In addition, a methodologist experienced in systematic review and guideline development consulted with the panel throughout the project.

\section{Conflict of Interest Policy}

Prior to acceptance on the expert or advisory panel, potential members completed a joint guideline conflict of interest (COI) disclosure process, whose policy and form (in effect July 2011) require disclosure of material financial interest in, or potential for benefit of significant value from, the guideline's development or its recommendations 12 months prior through the time of publication. The potential members completed the COI disclosure form, listing any relationship that could be interpreted as constituting an actual, potential, or apparent conflict. All project participants were required to disclose conflicts prior to beginning and continuously throughout the project's timeline. Disclosed conflicts of the expert panel members are listed in Appendix 1 and Appendix 2.

The ASCP, CAP, AMP, and ASCO provided funding for the administration of the project; no industry funds were used in the development of the guideline. All panel members volunteered their time and were not compensated for their involvement, except for the contracted methodologist. Please see the Supplemental Digital Content (SDC) at www.archivesofpathology.org in the May 2017 table of contents for full details on the COI policy.

\section{OBJECTIVE}

The scope of the project was to develop an evidencebased guideline to help establish standard molecular biomarker testing, guide targeted therapies, and advance personalized care for patients with CRC. The panel addressed the following key questions:

1. What biomarkers are useful to select patients with CRC for targeted and conventional therapies?

2. How should tissue specimens be processed for biomarker testing for CRC management?

3. How should biomarker testing for CRC management be performed?

4. How should molecular testing of CRC be implemented and operationalized?

5. Are there emerging genes/biomarkers that should be routinely tested in CRC? 


\section{MATERIALS AND METHODS}

This evidence-based guideline was developed following standards as endorsed by the Institute of Medicine. ${ }^{9}$ A detailed description of the methods and systematic review (including the quality assessment and complete analysis of the evidence) can be found in the SDC.

\section{Literature Search and Selection}

A comprehensive search for literature was performed in MEDLINE using the OvidSP (August 1, 2013) and PubMed (September 17, 2013) interfaces. The initial MEDLINE search encompassed the publication dates of January 1, 2008, through August 1, 2013 (OvidSP), and January 1, 2008, through September 17, 2013 (PubMed). A supplemental literature search was performed using Scopus (September 25, 2013) to identify relevant articles published between January 1, 2008, and September 25, 2013, in journals not indexed in MEDLINE. The literature search of the electronic databases involved two separate searches in each database, the first using Medical Subject Headings (MeSH) terms and keywords for the concepts "colorectal cancer," "biomarkers," "treatment," and "treatment outcomes" and the second using terms for the concepts "colorectal cancer," "biomarkers," and "laboratory methods." Limits were set for human studies published in English, and a publication filter was applied to exclude lower levels of evidence such as letters, commentaries, editorials, and case reports. The Ovid search was rerun on February 12, 2015, to identify articles published since August 1, 2013.

In addition to the searches of electronic databases, an Internet search of international health organizations, the National Guidelines Clearinghouse, and Guidelines International Network was conducted for existing relevant guidelines or protocols. Guidelines were included if they were published since 2008 in English. The proceedings of the meetings of the ASCO and ASCO-Gastrointestinal Cancers Symposium, European Society for Medical Oncology, and the American Association for Cancer Research from 2012 and 2013 were also searched for relevant abstracts.

A focused examination of all systematic reviews retrieved by the initial literature search and retained after full-text review was performed to identify primary research studies not already included. In addition, recommendations from the expert panel were reviewed, and the reference lists of all articles deemed eligible for inclusion were scanned for relevant reports. The results of all searches were combined and deduplicated.

Detailed information regarding the literature search strategy can be found in the SDC.

\section{Eligible Study Designs}

Practice guidelines, consensus documents, systematic reviews, meta-analyses, randomized controlled trials, comparative studies, reviews, and evaluation studies were eligible for inclusion. In addition to journal articles, the search identified meeting abstracts.

\section{Inclusion Criteria}

Published studies were selected for full-text review if they met each of the following criteria:

1. Patients with colorectal or rectal cancer with a pathology diagnosis of adenocarcinoma or adenocarcinoma with neuroendocrine differentiation, either primary or metastatic

2. Patients of all ages

3. Patients with cancer of any invasive stage (T1-T4)

4. Biomarker testing such as KRAS (Kirsten rat sarcoma viral oncogene homolog), DNA MMR/MSI, BRAF (V-raf murine sarcoma viral oncogene homolog B1), NRAS (neuroblastoma RAS viral [v-ras] oncogene homolog), PIK3CA (phosphatidylinositol-4,5-bisphosphate 3-kinase, catalytic subunit alpha),
PTEN (phosphatase and tensin homolog), MLH1 (MutL homolog 1) methylation, or gene expression profiles

5. Comparative studies

6. Human studies

7. Studies published in English

\section{Exclusion Criteria}

1. All other tumor primaries and types (ie, noncolorectal or nonrectal cancers, tumor types other than adenocarcinoma or adenocarcinoma with neuroendocrine differentiation)

2. Patients with noninvasive tumors (ie, intraepithelial, dysplasia, in situ, polyps without carcinoma)

3. Studies of colorectal cancers without biomarker testing, novel biomarkers-for example, VEG-F (vascular endothelial growth factor), XRCC1 (X-ray repair complementing defective repair in Chinese hamster cells 1), IGF (insulin-like growth factor), ERCC (excision repair cross-complementing rodent repair deficiency, complementation group 1), micro-RNA, TYMS (thymidylate synthetase), GCC (guanylyl cyclase C), LINE (long interspersed nucleotide element) methylation, CIMP (CpG island methylator phenotype), HER2 (V-erb-b2 erythroblastic leukemia viral oncogene homolog 2), CIN (chromosomal instability) status $\mathrm{LOH}$ (loss of heterozygosity), and germline (genetics only) testing

4. Non-English-language articles

5. Animal studies

6. Studies published prior to 2002

7. Noncomparative studies, letters, commentaries, or editorials

8. Studies that did not address at least one of the defined inclusion criteria

9. Studies with fewer than 50 patients per comparison arm

\section{Outcomes of Interest}

The primary outcomes of interest included survival outcomes and performance characteristics of laboratory testing assays. Survival outcomes included overall survival (OS), disease-free survival (DFS), progression-free survival (PFS), recurrence-free survival, time to recurrence, and response to therapy (eg, complete and partial response). Laboratory data and test performance characteristics included percent mutation, concordance of testing methods, sensitivity of testing methods, specificity of testing methods, concordance of detected mutations between primary and metastatic mutations (number [\%] of cases with mutations versus number of cases with no mutations in the gene of interest), and concordance of mutations (synchronous primary versus metastatic, metachronous primary versus metastatic, between synchronous metastases, between metachronous metastases).

\section{Quality Assessment}

An assessment of the quality of the evidence was performed for all retained studies following application of the inclusion and exclusion criteria by the methodologist. Using this method, studies deemed to be of low quality would not be excluded from the systematic review but would be retained and their methodologic strengths and weaknesses discussed where relevant. Studies would be assessed by confirming the presence of items related to both internal and external validity, which are all associated with methodologic rigor and a decrease in the risk of bias. The quality assessment of the studies was performed by determining the risk of bias by assessing key indicators, based on study design, against known criteria. (Refer to the SDC for detailed discussion of the quality assessment.)

For strength of the evidence, the panel considered the level of evidence, as well as its quantity and quality of included studies. The level of evidence was based on the study design as described in Table 1. ${ }^{10}$ In general, level I and II evidence is considered most appropriate to answer clinical questions, but in the absence of such high-quality evidence, the panel considered data from lower quality studies. The quantity of evidence refers to the 


\begin{tabular}{|cl|}
\hline & \multicolumn{1}{c|}{ Table 1. Levels of Evidence $^{\text {a }}$} \\
\hline Level & \multicolumn{1}{c|}{ Description } \\
\hline Level I & Evidence derived from systematic reviews of appropriate level II studies and/or clinical practice guidelines \\
Level II & Evidence derived from randomized controlled trials \\
Level III & Evidence derived from comparative studies (eg, prospective cohort studies, retrospective cohort studies) \\
Level IV & Evidence without a comparator (eg, case reports, case series, narrative reviews) \\
\hline
\end{tabular}

a Data derived from National Health and Medical Research Council. ${ }^{10}$

number of studies and number of cases included for each outcome in the recommendation. The quality of studies reflects how well the studies were designed to eliminate bias and threats to validity.

The appropriateness of the study design and data collected, relevance and clarity of findings, and adequacy of conclusions were evaluated. Each study was assessed individually (refer to the SDC for individual assessments and results) and then summarized by study type. Components such as generalizability and applicability were also considered when determining the strength of evidence. A summary of the overall quality of the evidence was given considering the evidence in totality. Ultimately, the designation (ie, rating or grade) of the strength of evidence is a judgment by the expert panel of its level of confidence that the evidence from the studies informing the recommendations reflects true effect. Table 2 describes the grades for strength of evidence. ${ }^{11}$

\section{Assessing the Strength of Recommendations}

Development of recommendations requires that the panel review the identified evidence and make a series of key judgments (using procedures described in the SDC). Grades for strength of recommendations were developed by the CAP Pathology and Laboratory Quality Center and are described in Table 3. ${ }^{11}$

\section{Guideline Revision}

This guideline will be reviewed every 4 years or earlier in the event of publication of substantive and high-quality evidence that could potentially alter the original guideline recommendations. If necessary, the entire panel will reconvene to discuss potential changes. When appropriate, the panel will recommend revision of the guideline to the ASCP, CAP, AMP, and ASCO for review and approval.

\section{Disclaimer}

Practice guidelines and consensus statements reflect the best available evidence and expert consensus supported in practice. They are intended to assist physicians and patients in clinical decision making and to identify questions and settings for further research. With the rapid flow of scientific information, new evidence may emerge between the time a practice guideline or consensus statement is developed and when it is published or read.
Guidelines and statements are not continually updated and may not reflect the most recent evidence. Guidelines and statements address only the topics specifically identified therein and are not applicable to other interventions, diseases, or stages of diseases. Furthermore, guidelines and consensus statements cannot account for individual variation among patients and cannot be considered inclusive of all proper methods of care or exclusive of other treatments. It is the responsibility of the treating physician or other health care provider, relying on independent experience and knowledge, to determine the best course of treatment for the patient. Accordingly, adherence to any practice guideline or consensus statement is voluntary, with the ultimate determination regarding its application to be made by the physician in light of each patient's individual circumstances and preferences. The ASCP, $\mathrm{CAP}, \mathrm{AMP}$, and ASCO make no warranty, express or implied, regarding guidelines and statements and specifically exclude any warranties of merchantability and fitness for a particular use or purpose. The ASCP, CAP, AMP, and ASCO assume no responsibility for any injury or damage to persons or property arising out of or related to any use of this statement or for any errors or omissions.

\section{RESULTS}

A total of 4,197 studies met the search term requirements. A total of 123 articles were included for data extraction. Excluded articles were available as discussion or background references. The panel convened 14 times (11 teleconference webinars and three face-to-face meetings) from July 27, 2013, through September 24, 2015, to develop the scope, draft recommendations, review and respond to solicited feedback, and assess the quality of evidence that supports the final recommendations. Additional work was completed via electronic mail. An open comment period was held from March 30, 2015, through April 22, 2015, during which draft recommendations were posted on the AMP website. Twenty-one guideline statements had an agreement ranging from $60 \%$ to $94 \%$ for each statement from the opencomment period participants (refer to Outcomes in the SDC for full details). The website received a total of 248

\begin{tabular}{|c|c|c|}
\hline Designation & Description & Quality of Evidence \\
\hline Convincing & $\begin{array}{l}\text { High confidence that available evidence reflects true effect. } \\
\text { Further research is very unlikely to change the confidence } \\
\text { in the estimate of effect. }\end{array}$ & High/intermediate quality of evidence \\
\hline Adequate & $\begin{array}{l}\text { Moderate confidence that available evidence reflects true } \\
\text { effect. Further research is likely to have an important } \\
\text { impact on the confidence in estimate of effect and may } \\
\text { change the estimate. }\end{array}$ & Intermediate/low quality of evidence \\
\hline Insufficient & $\begin{array}{l}\text { Evidence is insufficient to discern net effect. Any estimate of } \\
\text { effect is very uncertain. }\end{array}$ & $\begin{array}{l}\text { Insufficient evidence and expert panel } \\
\text { uses formal consensus process to reach } \\
\text { recommendation }\end{array}$ \\
\hline
\end{tabular}

${ }^{a}$ Adapted from Guyatt et al ${ }^{11}$ by permission from BMJ Publishing Group Limited. 


\begin{tabular}{|c|c|c|}
\hline Strong recommendation & $\begin{array}{l}\text { Recommend for or against a particular } \\
\text { molecular testing practice for colorectal } \\
\text { cancer (can include must or should) }\end{array}$ & $\begin{array}{l}\text { Supported by convincing or adequate strength of } \\
\text { evidence, high or intermediate quality of evidence, } \\
\text { and clear benefit that outweighs any harms }\end{array}$ \\
\hline Recommendation & $\begin{array}{l}\text { Recommend for or against a particular } \\
\text { molecular testing practice for colorectal } \\
\text { cancer (can include should or may) }\end{array}$ & $\begin{array}{l}\text { Some limitations in strength of evidence (adequate or } \\
\text { inadequate) and quality of evidence (intermediate or } \\
\text { low), balance of benefits and harms, values, or costs, } \\
\text { but panel concludes that there is sufficient evidence } \\
\text { and/or benefit to inform a recommendation }\end{array}$ \\
\hline Expert consensus opinion & $\begin{array}{l}\text { Recommend for or against a particular } \\
\text { molecular testing practice for colorectal } \\
\text { cancer (can include should or may) }\end{array}$ & $\begin{array}{l}\text { Serious limitations in strength of evidence (inadequate } \\
\text { of insufficient), quality of evidence (intermediate or } \\
\text { low), balance of benefits and harms, values, or costs, } \\
\text { but panel consensus is that a statement is necessary }\end{array}$ \\
\hline No recommendation & $\begin{array}{l}\text { No recommendation for or against a particular } \\
\text { molecular testing practice for colorectal } \\
\text { cancer }\end{array}$ & $\begin{array}{l}\text { Insufficient evidence or agreement of the balance of } \\
\text { benefits and harms, values, or costs to provide a } \\
\text { recommendation }\end{array}$ \\
\hline
\end{tabular}

${ }^{a}$ Data derived from Guyatt et al. ${ }^{11}$

comments. Teams of three to four expert panel members were assigned three to five draft recommendations to review all comments received and provide an overall summary to the rest of the panel. Following panel discussion and the final quality of evidence assessment, the panel members determined whether to maintain the original draft recommendation as is, revise it with minor language change, or consider it as a major recommendation change. The expert panel modified eight draft statements based on the feedback during the open-comment period and the considered judgment process. Resolution of all changes was obtained by majority consensus of the panel using nominal group technique (rounds of email discussion and multiple edited recommendations) among the panel members. The final recommendations were approved by the expert panel with a formal vote. The panel considered the risks and benefits throughout the whole process in their considered judgment process. Formal cost analysis or cost-effectiveness was not performed.

Each organization instituted a review process to approve the guideline. The ASCP assigned the review of the guideline to a Special Review Panel. For the CAP, an independent review panel (IRP) representing the Council on Scientific Affairs was assembled to review and approve the guideline. The IRP was masked to the expert panel and vetted through the COI process. The AMP approval process required the internal review of an independent panel led by the Publications and Communications Committee chair and Executive Committee approval. The ASCO approval process required the review and approval of the Clinical Practice Guidelines Committee.

\section{GUIDELINE STATEMENTS}

1. Recommendation.-Patients with $C R C$ being considered for anti-EGFR therapy must receive RAS mutational testing. Mutational analysis should include KRAS and NRAS codons 12 and 13 of exon 2, 59 and 61 of exon 3, and 117 and 146 of exon 4 ("expanded" or "extended" RAS) (Table $4)$.

Aberrant activation of EGFR signaling pathways in CRC is primarily associated with activating mutations of genes in the mitogen-activated protein kinase and phosphatidylinositol-3-kinase (PI3K) pathways. Together, KRAS, NRAS, and $B R A F$ mutations have been reported to occur in more than half of all CRC cases, and KRAS or NRAS and BRAF mutations are inversely associated, with a small proportion of individual CRCs showing co-occurrence of RAS and RAF mutations. ${ }^{3,12}$

Cetuximab and panitumumab are antibodies that bind to the extracellular domain of EGFR, blocking the binding of EGF and other EGFR endogenous ligands, thereby blocking EGFR signaling. Earlier studies reported the effects of antiEGFR antibody treatment independent of KRAS status. ${ }^{13-16}$ However, it was later reported that targeted EGFR therapies with cetuximab or panitumumab improve PFS and OS in patients with metastatic CRC with wild-type KRAS but not for patients with mutated KRAS., ${ }^{2,3,17}$ In these earlier studies, only mutations of KRAS exon 2 were considered. Based on the available clinical trial data in 2009, ASCO recommended that patients with metastatic CRC who are candidates for anti-EGFR antibody therapy should have their tumor tested for KRAS mutations in a Clinical Laboratory Improvements Amendments '88 (CLIA)-accredited laboratory. ${ }^{2}$

A large body of evidence was available to guide the recommendation in the current guideline for $R A S$ testing in colorectal cancers (Table 5 and Supplemental Table 14). From 2008 to 2015, there were 311 primary studies that included 74,546 patients and reported treatment outcomes for patients with RAS mutations compared with nonmutated/wild type. ${ }^{12-16,18-45}$ The most common comparison of anti-EGFR antibody treatment outcomes was between KRAS mutation versus KRAS nonmutated/wild type. ${ }^{18-20,22,24-26,28-31,33-42}$ Some studies also compared the effects of adding an anti-EGFR inhibitor to KRAS nonmutated/wild-type patients versus chemotherapy alone. ${ }^{18,22,24,26,28,36-38}$ A few studies reported anti-EGFR antibody treatment outcomes for the following comparisons: KRAS G13D versus codon 12 mutations, ${ }^{32}$ KRAS codon 13 mutations versus other mutations, ${ }^{21}$ and G13D versus other exon 2 mutations. ${ }^{23}$

The reported anti-EGFR therapy outcomes in these studies were pooled survival, ${ }^{13-16,21-27,29,32-37,39,41}$ pooled PFS, $13,15,16,18,21-27,29,31-36,39,41$ and pooled objective response rate (ORR). ${ }^{13,15,16,18,21,22,25,26,30-36,41}$ Thirteen studies reported significant differences between comparators. . $^{15,21,23-27,32,33,35-37,39}$ The systematic review literature of data on anti-EGFR therapy outcomes is presented in Supplemental Table 14. Five of these studies detected a significant pooled survival advantage of antiEGFR-treated patients for KRAS nonmutated/wild type compared with KRAS mutation. ${ }^{21,33,35,37,39}$ Three studies detected an advantage for patients with nonmutated tumors given antiEGFR treatment compared with KRAS mutation-positive 


\section{Guideline Statement}

1. Patients with colorectal carcinoma being considered for anti-EGFR therapy must receive $R A S$ mutational testing. Mutational analysis should include KRAS and NRAS codons 12 and 13 of exon 2, 59 and 61 of exon 3, and 117 and 146 of exon 4 ("expanded" or "extended" RAS).

2a. BRAF p.V600 (BRAF c.1799 [p.V600]) mutational analysis should be performed in colorectal cancer tissue in patients with colorectal carcinoma for prognostic stratification.

2b. BRAF p.V600 mutational analysis should be performed in deficient MMR tumors with loss of MLH1 to evaluate for Lynch syndrome risk. Presence of a BRAF mutation strongly favors a sporadic pathogenesis. The absence of a BRAF mutation does not exclude risk of Lynch syndrome.

3. Clinicians should order mismatch repair status testing in patients with colorectal cancers for the identification of patients at high risk for Lynch syndrome and/or prognostic stratification.

4. There is insufficient evidence to recommend BRAF c.1799 p.V600 mutational status as a predictive molecular biomarker for response to anti-EGFR inhibitors.

5. There is insufficient evidence to recommend PIK3CA mutational analysis of colorectal carcinoma tissue for therapy selection outside of a clinical trial.

Note: Retrospective studies have suggested improved survival with postoperative aspirin use in patients whose colorectal carcinoma harbors a PIK3CA mutation.

6. There is insufficient evidence to recommend PTEN analysis (expression by immunohistochemistry or deletion by fluorescence in situ hybridization) in colorectal carcinoma tissue for patients who are being considered for therapy selection outside of a clinical trial.

7. Metastatic or recurrent colorectal carcinoma tissues are the preferred specimens for treatment predictive biomarker testing and should be used if such specimens are available and adequate. In their absence, primary tumor tissue is an acceptable alternative and should be used.

8. Formalin-fixed, paraffin-embedded tissue is an acceptable specimen for molecular biomarker mutational testing in colorectal carcinoma. Use of other specimens (eg, cytology specimens) will require additional adequate validation, as would any changes in tissue-processing protocols.

9. Laboratories must use validated colorectal carcinoma molecular biomarker testing methods with sufficient performance characteristics for the intended clinical use. Colorectal carcinoma molecular biomarker testing validation should follow accepted standards for clinical molecular diagnostics tests.

10. Performance of molecular biomarker testing for colorectal carcinoma must be validated in accordance with best laboratory practices.

11. Laboratories must validate the performance of IHC testing for colorectal carcinoma molecular biomarkers (currently IHC testing for MLH1, MSH2, MSH6, and PMS2) in accordance with best laboratory practices.

12. Laboratories must provide clinically appropriate turnaround times and optimal utilization of tissue specimens by using appropriate techniques (eg, multiplexed assays) for clinically relevant molecular and immunohistochemical biomarkers of colorectal cancer.

13. Molecular and IHC biomarker testing in colorectal carcinoma should be initiated in a timely fashion based on the clinical scenario and in accordance with institutionally accepted practices.

Note: Test ordering can occur on a case-by-case basis or by policies established by the medical staff.

14. Laboratories should establish policies to ensure efficient allocation and utilization of tissue for molecular testing, particularly in small specimens.

15. Members of the patient's medical team, including pathologists, may initiate colorectal carcinoma molecular biomarker test orders in accordance with institutionally accepted practices.

16. Laboratories that require send-out of tests for treatment predictive biomarkers should process and send colorectal carcinoma specimens to reference molecular laboratories in a timely manner. Note: It is suggested that a benchmark of $90 \%$ of specimens should be sent out within 3 working days.

17. Pathologists must evaluate candidate specimens for biomarker testing to ensure specimen adequacy, taking into account tissue quality, quantity, and malignant tumor cell fraction. Specimen adequacy findings should be documented in the patient report.

18. Laboratories should use colorectal carcinoma molecular biomarker testing methods that are able to detect mutations in specimens with at least 5\% mutant allele frequency, taking into account the analytical sensitivity of the assay (limit of detection [LOD]) and tumor enrichment (eg, microdissection).

Note: It is recommended that the operational minimal neoplastic carcinoma cell content tested should be set at least two times the assay's LOD.

19. Colorectal carcinoma molecular biomarker results should be made available as promptly as feasible to inform therapeutic decision making, both prognostic and predictive.

Note: It is suggested that a benchmark of $90 \%$ of reports be available within 10 working days from date of receipt in the molecular diagnostics laboratory.

20. Colorectal carcinoma molecular biomarker testing reports should include a results and interpretation section readily understandable by oncologists and pathologists. Appropriate Human Genome Variation Society and Human Genome Organisation nomenclature must be used in conjunction with any historical genetic designations.

21. Laboratories must incorporate colorectal carcinoma molecular biomarker testing methods into their overall laboratory quality improvement program, establishing appropriate quality improvement monitors as needed to ensure consistent performance in all steps of the testing and reporting process. In particular, laboratories performing colorectal carcinoma molecular biomarker testing must participate in formal proficiency testing programs, if available, or an alternative proficiency assurance activity.
Strength of

Recommendation

Recommendation

Recommendation

Recommendation

Recommendation

No recommendation

No recommendation

No recommendation

Expert consensus opinion

Expert consensus opinion

Strong recommendation

Strong recommendation

Strong recommendation

Expert consensus opinion

Expert consensus opinion

Expert consensus opinion

Expert consensus opinion

Expert consensus opinion

Expert consensus opinion

Expert consensus opinion

Expert consensus opinion

Expert consensus opinion

Strong recommendation

EGFR, epidermal growth factor receptor; IHC, immunohistochemistry; MMR, mismatch repair; PTEN, phosphatase and tensin homolog. 
patients given chemotherapy alone. ${ }^{24,26,36}$ Twenty of the included studies pooled PFS, 13,15,16,18,21-27,29,31-36,39,41 with 19 reporting significant differences between comparators. ${ }^{13,15,18,21-27,29,31-36,39,41}$ Fourteen papers detected a significant PFS advantage for adding an anti-EGFR inhibitor to chemotherapy for KRAS nonmutated/wild-type patients compared with chemotherapy alone. ${ }^{13,15,18,22,24-26,29,31,33,34,36,39,41}$ Sixteen of the included papers pooled ORR, 13,15,16,18,21,22,25,26,30-36,41 with 14 reporting significant differences between comparators. ${ }^{15,18,21,22,25,26,30-36,41}$ Eight studies detected ORR advantages for adding an anti-EGFR inhibitor to chemotherapy for patients with nonmutated/wild-type tumors compared with chemotherapy alone, ${ }^{18,25,26,30,33,34,36,41}$ and four detected an ORR advantage for KRAS nonmutated/wild-type patients over mutation patients. ${ }^{22,31,32,35}$ Survival advantages (OS and PFS, ORR) for G13D mutations over codon 12 and G13D over other mutations were reported in two studies ${ }^{23,32}$ and codon 13 over other KRAS mutations. ${ }^{21}$

Recent studies showed conclusive evidence that in addition to mutations in KRAS exon 2, other RAS mutations in KRAS exons 3 and 4 and NRAS exons 2, 3, and 4 were also associated with nonresponse of metastatic CRC to antiEGFR monoclonal antibody therapy..$^{12,44,46}$ Douillard et $\mathrm{al}^{44}$ published a reanalysis of the Panitumumab Randomized Control Trial in Combination with Chemotherapy for Metastatic Colorectal Cancer to Determine Efficacy (PRIME) trial, reporting that patients with any RAS mutations were associated with inferior PFS and OS with panitumumabFOLFOX4 treatment, which was consistent with the findings previously reported for patients with KRAS mutations in exon 2. Subsequently, a meta-analysis of nine randomized clinical trials provided further evidence that not all KRAS exon 2 nonmutated/wild-type tumors benefit from anti-EGFR monoclonal antibody treatment in metastatic CRC. ${ }^{12}$ Patients with colorectal cancers that are KRAS exon 2 nonmutated/wild type but harbor RAS mutations in KRAS exons 3 and 4 or NRAS exons 2, 3, and 4 also have significantly inferior anti-EGFR treatment outcomes benefit compared with those without any RAS mutations (Table 5 and Table 6). RAS mutations occur mostly at exon 2, followed by mutations in exons 3 and 4 (Table 7). The results suggest that "extended" or "expanded" RAS mutation testing (KRAS exons 2, 3, and 4 and NRAS exons 2,3 , and 4) must be performed before the administration of an anti-EGFR monoclonal antibody therapy. ${ }^{12}$ In summary, current evidence indicates that both cetuximab and panitumumab should only be prescribed for patients with metastatic CRCs that are nonmutated/wild type for all known RAS-activating mutations. ${ }^{12}$

This recommendation is supported by 34 studies, ${ }^{12-16,18-45,47}$ comprising 29 systematic studies, ${ }^{12,13,15,16,18-22,24-42,47}$ two meta-analyses, ${ }^{14,23}$ one randomized controlled trial, ${ }^{44}$ one prospective cohort study, ${ }^{45}$ and one retrospective cohort study. ${ }^{43}$

Of the 29 systematic reviews, ${ }^{12,13,15,16,18-22,24-42,47}$ only three reported using a multidisciplinary panel, ${ }^{19,25,30}$ and only one reported taking patient preferences into account, ${ }^{37}$ although 13 examined important patient subtypes. ${ }^{12,15,16,18,21,22,24,27,30,33,37,39,40}$ All but one had welldescribed and reported methods sections. ${ }^{42}$ Seven did not report on conflict of interest. ${ }^{13,15,16,34,38,41,42}$ Only nine rated the quality of the included evidence, and these same nine were the only reviews that reported on the strength of the included evidence. ${ }^{16,18,21,22,24,25,32,37,39}$ None of the studies included a plan for updating. None of the systematic reviews reported industry funding, two reported no funding, ${ }^{16,31}$ and 11 did not report on the source of funding, if any..$^{13,15,26,32,34-36,38,41,42,47}$ Two of these systematic reviews were deemed to have a low risk of bias, ${ }^{24,37} 14$ were deemed to have a low to moderate risk of bias, $12,16,18,19,21,22,25,27,29,30,32,35,39,4712$ were deemed to have a moderate risk of bias, $13,15,20,26,28,31,33,34,36,38,40,41$ and one was deemed to have a high risk of bias. ${ }^{42}$

Of the two meta-analyses obtained, ${ }^{14,23}$ both had wellreported and reproducible methods sections, both described the planned pooling a priori, and both discussed the limitations of their analyses. Neither was based on a systematic review of the literature, and neither did a quality assessment of the included studies. One reported nonindustry funding, ${ }^{23}$ and the other reported industry funding. ${ }^{14}$ One was deemed to have a low to moderate risk of bias, ${ }^{23}$ and the other was deemed to have a moderate risk of bias. ${ }^{14}$

The single randomized controlled trial did not report on any details of the randomization, including blinding, the expected effect size and power calculation, and the length of follow-up. ${ }^{44}$ It did report on differences in baseline patient characteristics. This trial did report at least partial industry funding and was deemed to have a low to moderate risk of bias. ${ }^{44}$

The single prospective cohort study reported a balance between treatment and assessment groups, reported on baseline characteristics, and made adjustments in the analysis when differences were found. ${ }^{45}$ It reported nonindustry funding and was deemed to have a low risk of bias. ${ }^{45}$

The single retrospective cohort study reported that the treatment and assessment groups were in balance and also reported on baseline patient characteristics. ${ }^{43}$ It did not report that adjustments were made in the analysis to account for differences, where differences were found. This study reported nonindustry funding and was deemed to have a low risk of bias. ${ }^{43}$

All of the evidence that supported this recommendation was assessed, and none was found to have methodologic flaws that would raise concerns about their findings.

2a. Recommendation.-BRAF p.V600 (BRAF c.1799 [p.V600]) position mutational analysis should be performed in CRC tissue in selected patients with colorectal carcinoma for prognostic stratification.

$B R A F$ activating mutations occur in about $8 \%$ of advanced disease patients with CRC $^{47,48}$ and in approximately $14 \%$ of patients with localized stage II and III CRC., 8, As such, mutations in BRAF constitute a substantial subset of patients with CRC. The key questions related to $B R A F$ mutations are whether patients whose cancers carry a BRAF mutation have a poorer outcome compared with $B R A F$ mutation-negative tumors and whether the presence of a mutation predicts benefit from or lack thereof to anti-EGFR therapy.

Four systematic reviews $\mathrm{s}^{20,50-52}$ and three systematic reviews that included meta-analyse ${ }^{47,48,53}$ pertaining to the prognostic and predictive value of BRAF mutations in patients with $\mathrm{CRC}$ were identified through our systematic review process (Table 8 and Supplemental Table 14). These studies revealed that patients with advanced CRC who possess a BRAF mutation have significantly poorer outcomes as measured by PFS and OS and have a decreased response rate to anti-EGFR therapy relative to those with nonmutated BRAF. Poorer OS was also demonstrated for those patients with earlier stage II and III CRC having a $B R A F$ mutation $^{8,54}$; however, the poorer outcome appears to be primarily the result of decreased OS after relapse in these 
Table 5. KRAS Clinical Practice Guidelines, Systematic Reviews, Meta-Analyses, Prospective Cohort Studies, and Retrospective Cohort Studies

\begin{tabular}{|c|c|c|c|}
\hline Author, Year & $\begin{array}{l}\text { No. of Studies } \\
\text { (No. of Patients) }\end{array}$ & Comparison & Tests Used \\
\hline \multicolumn{4}{|c|}{ CPGs, systematic reviews, and meta-analyses on KRAS mutation + vs mutation- $(\mathrm{n}=30)$} \\
\hline Mao et al, ${ }^{32} 2013$ & $\begin{array}{l}\text { SR: } 10 \text { studies including } 1,487 \\
\text { patients with mCRC treated } \\
\text { with cetuximab }\end{array}$ & p.G13D vs codon 12 Mut+ & NR \\
\hline Jiang et al, ${ }^{27} 2013$ & $\begin{array}{l}\text { SR: } 13 \text { studies including } 1,174 \\
\text { patients with mCRC treated } \\
\text { with cetuximab or } \\
\text { panitumumab }\end{array}$ & $\begin{array}{l}\text { Increased vs not increased EGRF } \\
\text { GCN }\end{array}$ & $\mathrm{FISH}, \mathrm{CISH}, \mathrm{SISH}, \mathrm{qPCR}$ \\
\hline Chen et al, ${ }^{21} 2013$ & $\begin{array}{l}\text { SR: } 7 \text { studies including } 2,802 \\
\text { patients with mCRC }\end{array}$ & $\begin{array}{l}\text { Codon } 13 \text { Mut + vs other } \\
\text { mutations }\end{array}$ & PCR, direct sequencing \\
\hline Zhou et al, ${ }^{16} 2012$ & $\begin{array}{l}\text { SR: } 4 \text { RCTs including 1,270 first- } \\
\text { line patients with mCRC (all } \\
\text { Mut-) }\end{array}$ & $\begin{array}{l}\text { Oxaliplatin CT } \pm \text { anti-EGFR mAbs } \\
\text { Anti-EGFR }+ \text { CT vs CT }\end{array}$ & \\
\hline Zhang et al, ${ }^{41} 2011$ & $\begin{array}{l}\text { SR: } 4 \text { studies including } 2,912 \\
\text { patients with mCRC }\end{array}$ & Mut+ vs Mut- & NR \\
\hline Yang et $\mathrm{al}^{40} 2012$ & $\begin{array}{l}\text { SR: } 19 \text { studies including } 1,077 \\
\text { patients with mCRC }\end{array}$ & $\begin{array}{l}\text { Mut+ vs Mut- } \\
\text { GCN+ vs GCN- }\end{array}$ & $\mathrm{FISH}, \mathrm{qPCR}, \mathrm{CISH}$ \\
\hline Vale et al, ${ }^{39} 2012$ & $\begin{array}{l}\text { SR: } 10 \text { RCTs including 5,996 } \\
\text { patients with advanced CRC }\end{array}$ & Mut+ vs Mut- & NR \\
\hline Tsoukalas et al, ${ }^{38} 2012$ & $\begin{array}{l}\text { SR: } 13 \text { studies including 1,394 } \\
\text { patients with CRC }\end{array}$ & $\begin{array}{l}\text { Mut }+ \text { vs Mut- } \\
\text { Response to cetuximab vs no } \\
\text { response }\end{array}$ & NR \\
\hline Ross et $\mathrm{al}^{42} 2012$ & $\begin{array}{l}\text { SR: } 6 \text { studies including 2,526 } \\
\text { patients with mCRC }\end{array}$ & $\begin{array}{l}\text { Mut+ vs Mut- } \\
\text { Antibody vs control }\end{array}$ & $\begin{array}{l}\text { Sanger, pyrosequencing, PCR, } \\
\text { ARMS, Scorpion }\end{array}$ \\
\hline Ren et al, ${ }^{37} 2012$ & $\begin{array}{l}\text { SR: } 23 \text { studies including 1,362 } \\
\text { patients with mutations }(\sim 100 \% \\
\text { at codons } 12 \text { and } 13, \mathrm{n}=1 \text { at } \\
\text { codon } 61)\end{array}$ & Mut+ vs Mut- & $\mathrm{C}^{\mathrm{a}}$ \\
\hline Petrelli et al, ${ }^{34} 2012$ & $\begin{array}{l}\text { SR: } 4 \text { RCTs including } 484 \text { Mut- } \\
\text { patients with mCRC }\end{array}$ & $\begin{array}{l}\text { Mut+ vs Mut- } \\
\text { Cetuximab and/or panitumumab } \\
\quad+\text { CT vs CT alone }\end{array}$ & NR \\
\hline Modest et al, ${ }^{14} 2012$ & $\begin{array}{l}\text { M-A: } 3 \text { trials including } 119 \\
\text { patients with mCRC with codon } \\
12 \text { mutations vs other mutations }\end{array}$ & Cetuximab $\pm \mathrm{CT}$ & NR \\
\hline
\end{tabular}


Table 5. Extended

NR

G13D, 12$$
\begin{array}{r}
\mathrm{HR}, 0.52 ; 95 \% \mathrm{Cl}, 0.33-0.80 \\
P<.05, \text { in favor of G13D }
\end{array}
$$

NR

NR

13, other Mut+, Mut-

Exon 20

NR

Mut- only

NR

NR

$N R$

$12,13,61$

NR

12
Median, HR, 0.65;

95\% Cl, 0.46-0.92; $P<.05$, in favor of Mut-

$\mathrm{HR}, 0.52 ; 95 \% \mathrm{Cl}, 0.33-0.80$ $P<.05$, in favor of $\mathrm{Gl}$

Increased GCN associated with improved OS among patients treated with anti-EGFR mAbs (HR, 0.62; 95\% Cl, 0.50-0.77; $P<.05)$

Median, 9.5 months vs 4.8 months; HR, 0.55; 95\% Cl, 0.41-0.75, $P<.05$, in favor of cetuximab over BSC in Mut-

Panitumumab + BSC compared with BSC alone in Mut-, $P=\mathrm{ns}$

Median OS:

14.6 months, codon 13

11.8 months (other mutation)

17.3 months, Mut-

$\mathrm{HR}, 1.00 ; 95 \% \mathrm{Cl}, 0.88-1.13$,

$$
P=\mathrm{ns}
$$

Cetuximab + CT vs CT alone, Mut-:

$\mathrm{HR}, 0.84 ; 95 \% \mathrm{Cl}, 0.64-1.11$, $P=\mathrm{ns}$

Cetuximab + CT vs CT alone, mutation: $\mathrm{HR}, 1.03 ; 95 \% \mathrm{Cl}$, 0.74-1.44, $P=\mathrm{ns}$

No pooling due to statistical heterogeneity

NR

Third line, HR, 0.76; $95 \% \mathrm{Cl}$, $0.62-0.92, P<.05$

NR

$N R$

$\mathrm{HR}, 1.61 ; 95 \% \mathrm{Cl}, 1.19-2.18$, $P<.05$, in favor of treatment in Mut- vs Mut+ patients

$P=\mathrm{ns}$

$P=\mathrm{ns}$
Median PFS, HR, 0.85; 95\% Cl, $0.74-0.98 ; P<.05$, in favor of Mut-

PFS, HR, 0.54; 95\% Cl, 0.36$0.81, P<.05$, in favor of G13D

GCN associated with improved PFS (HR, 0.65; 95\% Cl, 0.47$0.89 ; P<.05)$

Median PFS, HR, 0.40; 95\% Cl, 0.30-0.54, $P<.05$, third-line cetuximab + BSC compared with BSC alone in Mut-

Median PFS, HR, 0.45; 95\% Cl, 0.34-0.59, $P<.05$, panitumumab + BSC compared with BSC alone in Mut-

Median PFS:

6.4 months, codon 13

4.1 months (other mutation)

6.6 months, Mut-

$\mathrm{HR}, 0.86 ; 95 \% \mathrm{Cl}, 0.71-1.04, P=$ $\mathrm{ns}$

Cetuximab + CT vs CT alone, Mut-: HR, 0.64; 95\% Cl, 0.50$0.84, P<.05$, favors + cetuximab

Cetuximab + CT vs CT alone, mutation: $\mathrm{HR}, 1.37 ; 95 \% \mathrm{Cl}$, $0.81-2.31, P=\mathrm{ns}$

No pooling due to statistical heterogeneity

First/second line, PFS, HR, 0.83; 95\% Cl, 0.76-0.90, $P<.05$

Third line, PFS, HR, 0.43; 95\% $\mathrm{Cl}, 0.35-0.52, P<.05$, in favor of anti-EGFR mAbs for Mutonly

NR

NR

NR

PFS, HR, 0.68, $P<.05$, in favor of adding cetuximab and/or panitumumab to CT in Mutpatients
NR

$N R$

NR

KRAS Mut+:

$48.3 \%$ vs KRAS Mut-: $54.8 \%$

(OR, 1.42; $95 \% \mathrm{Cl}, 1.05-$

$1.92 ; P<.05)$

KRAS pG13D: $22 \%$

KRAS 12: $16 \%$

KRAS Mut-: $44 \%$

(pG13D vs 12: RR, 1.64; 95\%

Cl, 1.13-2.38; $P<.05)$

pG13D vs Mut-: RR, 0.54; Cl,

$0.38-0.77 ; P<.05)$ NR

KRAS Mut-: $12.8 \%$

KRAS Mut+:

$1.2 \%, P<.05$, cetuximab + BSC compared with BSC alone in Mut-

KRAS Mut-: $10 \%$

KRAS Mut+: $0, P<.05$, panitumumab + BSC compared with BSC alone in Mut-

Codon 13 Mut+

vs other mutations: RR, 1.52 (95\% Cl, 1.10-2.09, $P<.05)$

Codon 13 Mut+ vs Mut-: RR, $0.61(95 \% \mathrm{Cl}, 0.45-0.83, P<$ $.05)$

RR, $1.08 ; 95 \% \mathrm{Cl}, 0.86-1.36, P$ $=\mathrm{ns}$

Cetuximab + CT vs CT alone: $\mathrm{RR}, 1.93 ; 95 \% \mathrm{Cl}, 1.14-3.26$, $P<.05$, favors + cetuximab

Cetuximab+CT vs CT alone, Mut-: RR, $1.44 ; 95 \% \mathrm{Cl}$, 1.20-1.73, $P<.05$, favors + cetuximab

No pooling due to statistical heterogeneity

NR

NR
NR

RR, 1.67, $P<.05$, in favor of adding cetuximab and/or panitumumab to CT in Mutpatients 
Table 5. Continued

\begin{tabular}{|cc}
\hline Author, Year & $\begin{array}{c}\text { No. of Studies } \\
\text { (No. of Patients) }\end{array}$
\end{tabular}

Loupakis et al, ${ }^{31} 2012$ SR: 8 trials including 6,609 patients with mCRC

\section{Comparison}

Mut+ vs Mut-

Ku et al, ${ }^{28} 2012$

SR: 2 RCTs including 261 patients with $\mathrm{mCRC}$

SR: 7 trials including 5,212 patients with advanced CRC, KRAS Mut- only

Mao et al, ${ }^{33} 2012$

Lin et al, ${ }^{29} 2011$

Ibrahim et al, ${ }^{13} 2011$

Dahabreh et al, ${ }^{22} 2011$

SR: 13 studies including 576 patients with $\mathrm{mCRC}$, all KRAS Mut-treated with anti-EGFR mAbs

SR: 8 studies including 5,325 patients with advanced CRC

SR: 4 studies including 2,115 patients with mCRC with MutKRAS

SR: 29 poolable studies including 5,032 patients with $\mathrm{mCRC}$ treated with anti-EGFR mAbs Mut+ vs Mut-

Baas et $\mathrm{al}^{20}{ }^{20} 2011$

Adelstein et al, ${ }^{18} 2011$

Qiu et $\mathrm{al}^{36} 2010$ Health Quality Ontario, ${ }^{24}$
2010 patients with advanced CRC

Ibrahim et al, ${ }^{26} 2010$

De Roock et al, ${ }^{23} 2010$

Allegra et al, ${ }^{19} 2009$

Linardou et al, ${ }^{30} 2008$
SR: 14 observational studies in

SR: 10 studies including 2,703 patients with $\mathrm{mCRC}$

M-A: 7 studies including 774 patients with mCRC who received cetuximab-based

treatment $\pm \mathrm{CT}$

SR: 21 studies including $\sim 1,213$ patients with mCRC (one study, with anti-EGFR mAbs

SR: 22 studies including 2,188 patients with $\mathrm{mCRC}$

SR: 5 RCTs including 627 patients with $\mathrm{mCRC}$ and 5 single-arm studies including 247 patients

SR: 8 studies including 817 patients with mCRC (306 with KRAS mutations)
Cetuximab $+5 \mathrm{FU}$ with oxaliplatin vs irinotecan Mut+ vs Mut-
Cetuximab or panitumumab $+\mathrm{CT} \quad \mathrm{NR}$ vs BSC

Mut+ vs Mut-

Direct sequencing, survey analysis, alleic discrimination, Sanger

Mut+ vs Mut-

NR

Panitumumab-based treatment vs control

NR

Mut+ vs Mut-

Cetuximab or panitumumab + CT vs CT alone

Concordance between KRAS Mut+/Mut-

between primary and metastases

Mut+ vs Mut-

Cetuximab or panitumumab $+\mathrm{CT}$ vs CT alone

Mut+ vs Mut-

Cetuximab + CT vs CT alone

Sequencing, pyrosequencing, PCR-RFLP, SSCP, AS-PCR, ASO

NR

DS, surveyor analysis, qPCR, $A D$, melting curve analysis

Mut+ vs Mut-

Cetuximab or panitumumab $+\mathrm{CT}$ vs CT alone

Mut+ vs Mut-

Cetuximab $+\mathrm{CT}$ vs CT alone

NR

pG13D vs other mutation

NR

Mut+ vs Mut-

PCR, direct sequencing

Mut+ vs Mut-

NR 
Table 5. Continued, Extended

\begin{tabular}{|c|c|c|c|}
\hline Codons Studied & OS & PFS & ORR \\
\hline NR & NR & $\begin{array}{l}\text { PFS, } \mathrm{HR}, 0.91 ; 95 \% \mathrm{Cl}, 0.84- \\
0.99 ; P<.05 \text {, in favor of } \\
\text { adding anti-EGFR mAbs to CT } \\
\text { in Mut- patients (irinotecan } \\
\text { favoring } \mathrm{CT}, P<.05 \text { ) }\end{array}$ & $\begin{array}{l}\mathrm{RR}, 1.17 ; 95 \% \mathrm{Cl}, 1.04-1.33 ; P \\
\quad<.05 \text {, in favor of KRAS Mut- }\end{array}$ \\
\hline NR & $\begin{array}{l}\text { No pooling performed in this } \\
\text { comparison } \\
\text { AIO trial, } P=\mathrm{ns} \\
\text { CECOG trial, } P<.05 \text { in favor of } \\
\text { cetuximab + FOLFOX in Mut- } \\
\text { patients }\end{array}$ & $\begin{array}{l}\text { No pooling performed in this } \\
\text { comparison } \\
\text { AIO trial, } P=\mathrm{ns} \\
\text { CECOG trial, } P=\mathrm{ns} \text { in favor of } \\
\text { cetuximab + FOLFOX in Mut- } \\
\text { patients }\end{array}$ & NR \\
\hline NR & $\begin{array}{l}\mathrm{HR}, 0.84 ; 95 \% \mathrm{Cl} \text {, in favor of } \\
0.73-0.98, P<.05 \text {, } \\
\text { anti-EGFR mAbs vs no mAbs in } \\
\text { Mut-patients }\end{array}$ & $\begin{array}{l}\text { PFS, HR, } 0.65 ; 95 \% \text { Cl } 0.51-0.83 \\
P<.05 \text {, in favor of anti-EGFR } \\
\text { mAbs vs no mAbs in Mut- } \\
\text { patients }\end{array}$ & $\begin{array}{c}\mathrm{RR}, 1.69 ; 95 \% \mathrm{Cl}, 1.20-2.38 ; P \\
<.05 \text {, in favor of anti-EGFR }\end{array}$ \\
\hline NR & $P=\mathrm{ns}$ & $\begin{array}{l}\text { PFS, HR, } 0.66 ; 95 \% \mathrm{Cl}, 0.53- \\
0.82, P<.05, \text { in favor of } \\
\text { adding anti-EGFR to CT in Mut- } \\
\text { patients }\end{array}$ & NR \\
\hline NR & $P=\mathrm{ns}$ & $\begin{array}{l}\text { PFS, HR, } 0.58 ; 95 \% \mathrm{Cl}, 0.36- \\
0.93 ; P<.05, \text { in favor of } \\
\text { adding panitumumab to CT in } \\
\text { Mut- patients }\end{array}$ & $\begin{array}{l}\text { OR, } 1.08 ; 95 \% \mathrm{Cl}, 0.75-1.58 ; P \\
\quad=\mathrm{ns}\end{array}$ \\
\hline NR & $\begin{array}{l}\mathrm{HR}, 1.30 ; 95 \% \mathrm{Cl}, 0.95-1.78 \\
P=\text { ns in Mut- patients }\end{array}$ & $\begin{array}{l}\text { PFS, } \mathrm{HR}, 2.22 ; 95 \% \mathrm{Cl}, 1.74- \\
2.84, P<.05 \text {, in favor of anti- } \\
\text { EGFR }+\mathrm{CT} \text { in Mut- patients } \\
\text { only }\end{array}$ & $\begin{array}{l}\text { Positive likelihood ratio, } 7.35 \\
(95 \% \mathrm{Cl}, 3.72-14.50) \\
\text { Negative likelihood ratio, } 0.55 \\
(95 \% \mathrm{Cl}, 0.49-0.61) \\
\text { KRAS mutations associated with } \\
\text { higher likelihood of response } \\
\text { failure }\end{array}$ \\
\hline $12,13,61$ & NR & $\begin{array}{l}\text { PFS, HR, } 0.80 ; 95 \% \mathrm{Cl}, 0.64- \\
0.99, P<.05 \text {, in favor of anti- } \\
\text { EGFR mAbs in Mut- patients }\end{array}$ & $\begin{array}{l}\mathrm{RD}, 15 \% ; 95 \% \mathrm{Cl}, 8 \%-22 \%, P \\
\quad<.05 \text {, in favor of } K R A S \text { Mut- } \\
+ \text { anti-EGFR treatment }\end{array}$ \\
\hline Exon 1,2 & $\begin{array}{l}\text { Median OS, } 6.9 \text { vs } 13.5 \text { months, } \\
\text { HR, } 2.17 ; 95 \% \mathrm{Cl}, 1.72-2.74, P \\
<.05, \text { longer median survival } \\
\text { shown in Mut- patients who } \\
\text { received anti-EGFR mAbs }+\mathrm{CT}\end{array}$ & $\begin{array}{l}\text { Median PFS, } 3.0 \text { vs } 5.8 \text { months, } \\
\text { HR, 1.94; } 95 \% \mathrm{Cl}, 1.62-2.33 ; P \\
<.05 \text {, longer median PFS } \\
\text { shown in Mut- patients who } \\
\text { received anti-EGFR mAbs }+ \text { CT }\end{array}$ & $\begin{array}{l}\text { KRAS Mut-: } 39 \% \\
\text { KRAS Mut+: } 14 \% \\
\text { RR, } 0.24 ; 95 \% \mathrm{Cl}, 0.16-0.38, P \\
\quad<.05\end{array}$ \\
\hline NR & $\begin{array}{l}\text { Mean } \mathrm{OS}, \mathrm{MD},-4.11 ; 95 \% \mathrm{Cl} \text {, } \\
-5.60 \text { to }-2.62, P<.05 \\
\text { longer survival detected in } \\
\text { Mut- patients treated with } \\
\text { cetuximab +irinotecan }\end{array}$ & $\begin{array}{l}\text { Mean PFS, MD, }=-3.32 ; 95 \% \mathrm{Cl} \text {, } \\
-4.86 \text { to }-1.78, P<.05 \\
\text { longer duration detected in } \\
\text { Mut- patients treated with } \\
\text { cetuximab + irinotecan }\end{array}$ & NR \\
\hline NR & $\begin{array}{l}P<.05 \text {, in favor of treatment with } \\
\text { cetuximab }+ \text { CT in Mut- } \\
\text { patients }\end{array}$ & $\begin{array}{l}\text { PFS, } P<.05, \text { in favor of treatment } \\
\text { with cetuximab }+C T \text { in Mut- } \\
\text { patients }\end{array}$ & $\begin{array}{l}\mathrm{OR}, 2.10 ; 95 \% \mathrm{Cl}, 1.42-3.10, P \\
\quad<.05\end{array}$ \\
\hline pG13D, 13 & $\begin{array}{l}\text { Median }(95 \% \mathrm{Cl}) \text { : } \\
\text { pG13D: } 7.6 \text { months }(5.7-20.5) \\
\text { Other mutations: } 5.7 \text { months }(4.9- \\
\quad 6.8) \\
\text { Mut-: } 10.1 \text { months }(9.4-11.3) \\
P<.05 \text {, pG13D superior to other } \\
\text { mutations }\end{array}$ & $\begin{array}{l}\text { Median }(95 \% \mathrm{Cl}) \text { PFS: } \\
\text { pG13D: } 4.0 \text { months }(1.9-6.2) \\
\text { Other mutations: } 1.9 \text { months }(1.8- \\
2.8) \\
\text { Mut-: } 4.2 \text { months }(3.9-5.4) \\
P<.05 \text {, pG13D superior to other } \\
\text { mutations }\end{array}$ & NR \\
\hline 12,13 & No pooling was performed & No pooling was performed & No pooling was performed \\
\hline $12,13,61$ & NR & NR & \\
\hline
\end{tabular}


Table 5. Continued

\begin{tabular}{|c|c|c|c|}
\hline Author, Year & $\begin{array}{l}\text { No. of Studies } \\
\text { (No. of Patients) }\end{array}$ & Comparison & Tests Used \\
\hline Sorich et al, ${ }^{12} 2015$ & $\begin{array}{l}\text { SR: } 9 \text { RCTs including } 5,948 \\
\text { patients with mCRC }\end{array}$ & $\begin{array}{l}\text { Mut+ vs Mut- } \\
\text { Anti-EGFR mAb treatment effect } \\
\text { size between RAS subgroups, } \\
\text { including Mut+ vs Mut- }\end{array}$ & $\begin{array}{l}\text { Bidirectional Sanger } \\
\text { sequencing, pyrosequencing, } \\
\text { MALDI-TOF analysis, and } \\
\text { WAVE-based Surveyor } \\
\text { analysis }\end{array}$ \\
\hline \multicolumn{4}{|c|}{ Randomized controlled trials $(\mathrm{n}=1)$} \\
\hline Douillard et al, ${ }^{44} 2013$ & $\begin{array}{l}\text { RCT: reanalysis of PRIME trial } \\
\text { (NCT: 00364013) data, } \\
\text { including 1,060 patients }\end{array}$ & $\begin{array}{l}\text { RAS Mut } \pm \text { and FOLFOX } 4 \pm \text { anti- } \\
\text { EGFR mAb }\end{array}$ & PCR, Sanger, Surveyor \\
\hline \multicolumn{4}{|c|}{ Prospective cohort studies $(n=1)$} \\
\hline Etienne-Grimaldi et al, & 251 patients & KRAS Mut+ vs KRAS Mut- & NR \\
\hline \multicolumn{4}{|c|}{ Retrospective cohort studies $(\mathrm{n}=1)$} \\
\hline Bando et al, ${ }^{43} 2013$ & 82 samples from 376 patients & $\begin{array}{l}\text { All Mut- vs } K R A S 12,13 \text { vs } K R A S \\
\quad 61,146\end{array}$ & $\begin{array}{l}\text { Luminex xMAP vs DS } \\
\quad \text { (concordance rate } 100 \%)\end{array}$ \\
\hline
\end{tabular}

5FU, fluorouracil; AD, allelic discrimination; AIO, German AIO colorectal study group; ARMS, amplification refractory mutation system; AS-PCR, allele-specific polymerase chain reaction; ASO, allele-specific oligonucleotide; $B R A F$, proto-oncogene B-Raf/v-Raf murine sarcoma viral oncogene homolog B; BSC, best supportive care; CECOG, Central European Cooperative Oncology Group; Cl, confidence interval; CISH, chromogenic in situ hybridization; CPG, clinical practice guideline; CRC, colorectal cancer; CT, chemotherapy; DS, direct sequencing; EGFR, epidermal growth factor receptor; FISH, fluorescence in situ hybridization; FOLFOX, folinic acid (leucovorin calcium), 5-fluorouracil, and oxaliplatin; FOLFOX4, folacin, 4fluorouracil, oxaliplatin; GCN, gene copy number; HR, hazard ratio; HTA, health technology assessment; KRAS, Kirsten rat sarcoma viral oncogene homolog; M-A, meta-analysis; mAbs, monoclonal antibodies; MALDI-TOF, matrix-assisted laser desorption/ionization-time of flight; mCRC, metastatic colorectal cancer; MD, mean difference; Mut-, mutation negative or wild type; Mut+, mutation positive; NR, not reported; NRAS, neuroblastoma RAS viral (v-ras) oncogene homolog; ns, nonsignificant; OR, odds ratio; ORR, objective response rate; OS, overall survival; PCR, polymerase chain reaction; PCR-RFLP, polymerase chain reaction-restriction fragment length polymorphism; PFS, progression-free survival; PIK3CA, phosphatidylinositol-4,5-bisphosphate 3-kinase catalytic subunit alpha; PRIME, Panitumumab Randomized Control Trial in Combination with Chemotherapy for Metastatic Colorectal Cancer to Determine Efficacy; PTEN, phosphatase and tensin homolog; qPCR, quantitative polymerase chain reaction; RCT, randomized controlled trial; $R A S$, rat sarcoma viral oncogene homolog; $R D$, risk difference; RFS, recurrence-free survival; RR, response rate; $\mathrm{SISH}$, silver in situ hybridization; SR, systematic review; SSCP, single-strand conformation polymorphism; xMAP, multiplex assay.

a Tests used by Ren et $\mathrm{al}^{37}$ : hybridization, $\mathrm{PCR}$, direct sequencing, topographic genotyping, AS-PCR, tissue transglutaminase enzyme, highperformance liquid chromatography, pyrosequencing, capillary sequencing.

patients rather than a harbinger of an increased rate of relapse. Finally, while outcomes in advanced disease patients with BRAF mutations were poorer relative to nonmutation patients, the data were consistent with a modest beneficial impact from the use of anti-EGFR agents relative to those patients whose tumors contained a $R A S$ mutation. ${ }^{55}$ In summary, patients with CRC that contains a $B R A F$ mutation have a worse outcome relative to nonmutation patients. Selected patients for BRAF mutation testing include patients with metastatic disease, since these patients have particularly poor outcomes. It is important to know the BRAF c.1799 (p.V600) mutation status of a

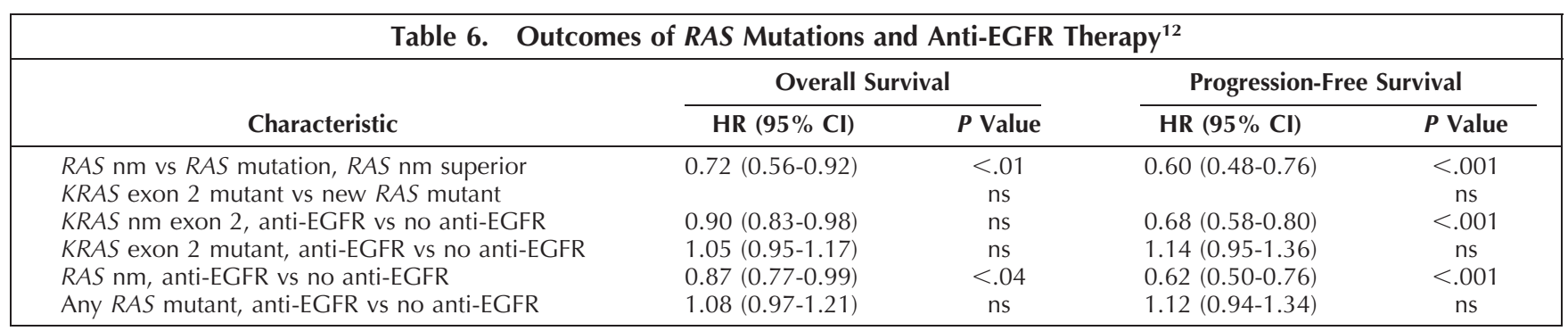

$\mathrm{Cl}$, confidence interval; EGFR, epidermal growth factor receptor; $\mathrm{HR}$, hazard ratio; KRAS, Kirsten rat sarcoma viral oncogene homolog; nm, nonmutated; ns, nonsignificant; $R A S$, rat sarcoma viral oncogene homolog. 


\begin{tabular}{|c|c|c|c|}
\hline Codons Studied & OS & PFS & ORR \\
\hline $\begin{array}{l}\text { KRAS/NRAS 12, 13, 59, } \\
\quad 61,117,146\end{array}$ & 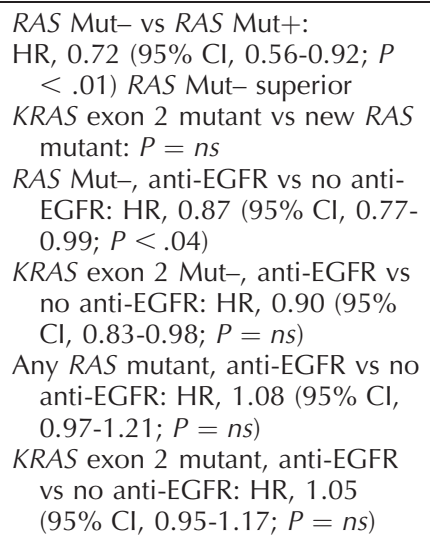 & 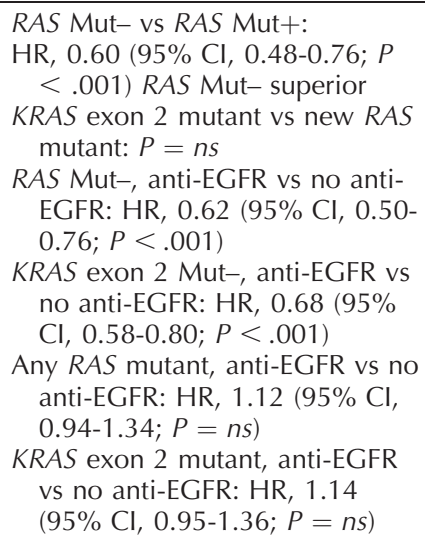 & NR \\
\hline $\begin{array}{l}\text { KRAS/NRAS 12, 13, 61, } \\
\quad 117,146\end{array}$ & $\begin{array}{l}\text { Mut } \pm \text { and anti-EGFR mAb } \pm \text { : } \\
26 \text { months vs } 20.2 \text { months } \\
\mathrm{HR}, 0.78 \text { (95\% Cl, 0.62-0.99; } P \\
<.05) \text { in favor of Mut- and + } \\
\text { anti-EGFR mAb }\end{array}$ & $\begin{array}{l}\text { Mut } \pm \text { and anti-EGFR mAb } \pm \text { : } \\
10.1 \text { months vs } 7.9 \text { months } \\
\mathrm{HR}, 0.72(95 \% \mathrm{Cl}, 0.58-0.90 ; P \\
<.05) \text { in favor of Mut- and }+ \\
\text { anti-EGFR mAb }\end{array}$ & NR \\
\hline KRAS 12, 13 & NR & $\begin{array}{l}\mathrm{RR}, 2.40(95 \% \mathrm{Cl}, 1.27-4.55 ; P \\
<.05) \text {, RFS shorter in KRAS } \\
\text { Mut+ patients with stage III } \\
\text { tumors }\end{array}$ & NR \\
\hline KRAS $12,13,61,146$ & $\begin{array}{l}\text { All Mut-: } 13.8 \text { months }(9.2-18.4) \\
\text { vs KRAS Mut+: } 8.2 \text { months } \\
(5.7-10.7 ; P<.05)\end{array}$ & $\begin{array}{l}\text { All Mut-: } 6.1 \text { months }(3.1-9.2) \text { vs } \\
\text { KRAS Mut+: } 2.7 \text { months }(1.2- \\
\quad 4.2 ; P<.05)\end{array}$ & $\begin{array}{c}\text { All Mut-: } 38.8 \% \text { vs KRAS } \\
\text { Mut+: } 4.8 \%, P<.05\end{array}$ \\
\hline
\end{tabular}

patient's CRC since standard therapy is not adequate for patients with metastatic disease and BRAF mutation. For these patients, some studies suggest the use of FOLFIRINOX (folinic acid [leucovorin calcium], 5-fluorouracil, irinotecan hydrochloride, and oxaliplatin) as first-line therapy, followed by enrollment in a clinical trial. ${ }^{56}$ Furthermore, early clinical trials data suggest that the combination of a BRAF plus EGFR inhibitor appears to be

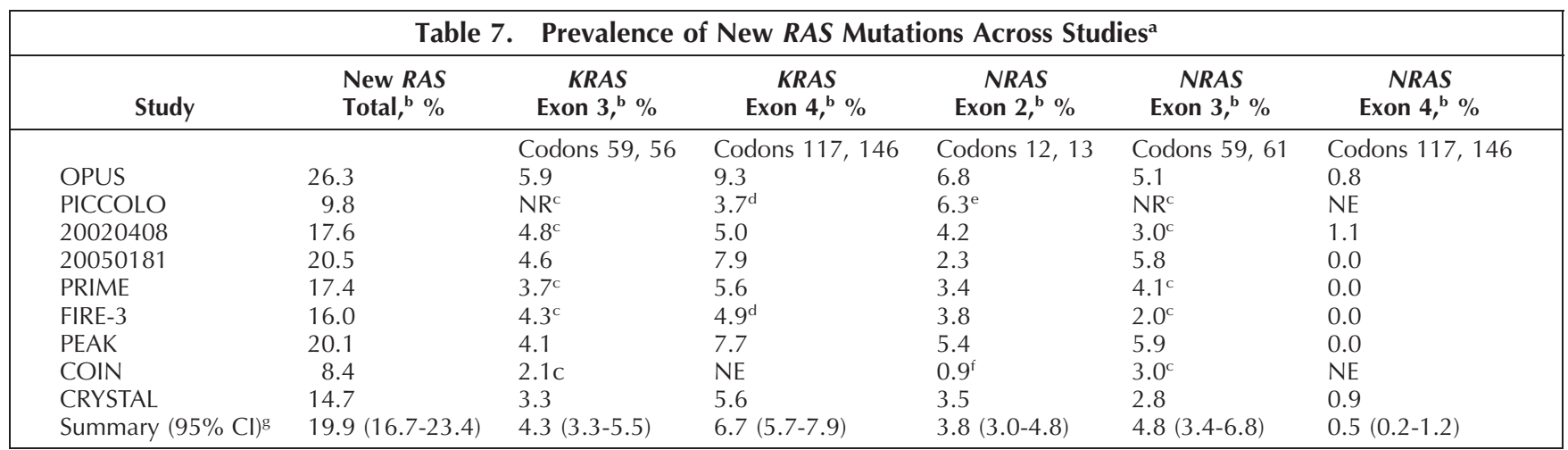

$\mathrm{Cl}$, confidence interval; COIN, Combination Chemotherapy With or Without Cetuximab as First-Line Therapy in Treating Patients With Metastatic Colorectal Cancer Trial; CRYSTAL, Cetuximab Combined with Irinotecan in First-Line Therapy for Metastatic Colorectal Cancer Trial; FIRE-3, Folinic Acid and Irinotecan (FOLFIRI) Plus Cetuximab vs FOLFIRI Plus Bevacizumab in First-Line Treatment Colorectal Cancer (CRC) Trial; NE, not evaluated; NR, evaluated but not reported; OPUS, Effect of Roflumilast on Exacerbation Rate in Patients With Chronic Obstructive Pulmonary Disease (BY217/M2-111) Trial; PEAK, Panitumumab Plus mFOLFOX6 vs Bevacizumab Plus mFOLFOX6 for First-Line Treatment of Metastatic Colorectal Cancer (mCRC) Patients With Wild-Type Kirsten Rat Sarcoma-2 Virus (KRAS) Tumors Trial; PICCOLO, Panitumumab and Irinotecan vs Irinotecan Alone for Patients With KRAS Wild-Type, Fluorouracil-Resistant Advanced Colorectal Cancer Trial; PRIME, Panitumumab Randomized Trial in Combination With Chemotherapy for Metastatic Colorectal Cancer to Determine Efficacy Trial.

a Modified from Sorich et $\mathrm{a}^{12}$ by permission of Oxford University Press on behalf of the European Society for Medical Oncology.

${ }^{\mathrm{b}}$ New RAS mutations are reported as a proportion of the KRAS exon 2 nonmutated/wild-type group.

${ }^{\mathrm{C}}$ KRAS and NRAS codon 59 mutation was not evaluated.

d KRAS codon 117 mutation was not evaluated.

e Exon 3 codon 61 mutations in addition to the exon 2 mutations.

${ }^{\mathrm{f}}$ Only NRAS mutation G12C evaluated.

g Random-effects meta-analysis summary estimates. 
Table 8. BRAF Clinical Practice Guidelines, Systematic Reviews, Meta-Analyses, Prospective Cohort Studies, and Retrospective Cohort Studies

\begin{tabular}{|c|c|c|c|}
\hline Author, Year & Study Type and Evidence & Comparison & Tests Used \\
\hline \multicolumn{4}{|c|}{ CPGs, systematic reviews, and meta-analyses $(\mathrm{n}=8)$} \\
\hline Parsons et al, ${ }^{52} 2012$ & $\begin{array}{l}\text { SR: } 36 \text { studies including 4,562 } \\
\text { CRC tumors }(B R A F), 43 \text { studies } \\
\text { including 2,975 CRC tumors } \\
(\text { MLH1) }\end{array}$ & Correlation study & NR \\
\hline Mao et al, ${ }^{51} 2011$ & $\begin{array}{l}\text { SR: } 11 \text { studies including } 1,046 \\
\text { patients with mCRC }\end{array}$ & Mut+ vs Mut- & $N R$ \\
\hline Lin et al, ${ }^{50} 2011$ & $\begin{array}{l}\text { SR: } 1 \text { study of } 649 \text { patients with } \\
\text { mCRC, all KRAS Mut-; } 6.5 \% \\
\text { were BRAF Mut+ }\end{array}$ & Mut+ vs Mut- & NR \\
\hline Baas et al, ${ }^{20} 2011$ & $\begin{array}{l}\text { SR: } 7 \text { studies including } 538 \\
\text { patients with mCRC }\end{array}$ & Mut+ vs Mut- & $\begin{array}{l}\text { Sequencing, } \\
\text { pyrosequencing }\end{array}$ \\
\hline Cui et al, ${ }^{53} 2014$ & $\begin{array}{l}\text { SR: } 4 \text { studies including 1,245 } \\
\text { patients }\end{array}$ & $\begin{array}{l}\text { Mut }+ \text { vs Mut- } \\
\text { CT } \pm \text { anti-EGFR mAbs }\end{array}$ & PCR \\
\hline
\end{tabular}

Yang et $\mathrm{al}^{71} 2013$

Yuan et $\mathrm{al}^{, 48} 2013$

Xu et $\mathrm{al}^{47} 2013$

Prospective cohort studies $(n=1)$

Etienne-Grimaldi et al, ${ }^{45} 2014$

Retrospective cohort studies $(\mathrm{n}=1)$

Bando et al, ${ }^{43} 2013$

82 samples from 376 patients
SR: 21 studies including 5,229 patients

SR: 19 studies including 2,875 patients

251 patients
All Mut- vs BRAF Mut+ and PIK3CA Mut-
Mut+ vs Mut-

Mut+ vs Mut-

Mut+ vs Mut-

NR

$B R A F$, proto-oncogene B-Raf/v-Raf murine sarcoma viral oncogene homolog B; $\mathrm{Cl}$, confidence interval; CPG, clinical practice guideline; CRC, colorectal cancer; $\mathrm{CT}$, chemotherapy; DS, direct sequencing; EGFR, epidermal growth factor receptor; HR, hazard ratio; KRAS, Kirsten rat sarcoma viral oncogene homolog; mAbs, monoclonal antibodies; mCRC, metastatic colorectal cancer; MLH1, mutL homolog 1; Mut-, mutation negative or wild type; Mut+, mutation positive; NR, not reported; $n s$, nonsignificant; ORR, objective response rate; OS, overall survival; PCR, polymerase chain reaction; PFS, progression-free survival; PIK3CA, phosphatidylinositol-4,5-bisphosphate 3-kinase catalytic subunit alpha; RR, response rate; RFS, recurrence-free survival; SR, systematic review; xMAP, multiplex assay.

a Yang et $\mathrm{al}^{71}$ : adenovirus-PCR pyrosequencing, allele-specific PCR, DS, PCR amplification, quantitative PCR, Sanger, real-time PCR, genotyping + DS, PCR clamping, melting curve analysis, DNA sequencing, and Taqman single-nucleotide polymorphism assay. 
Table 8. Extended

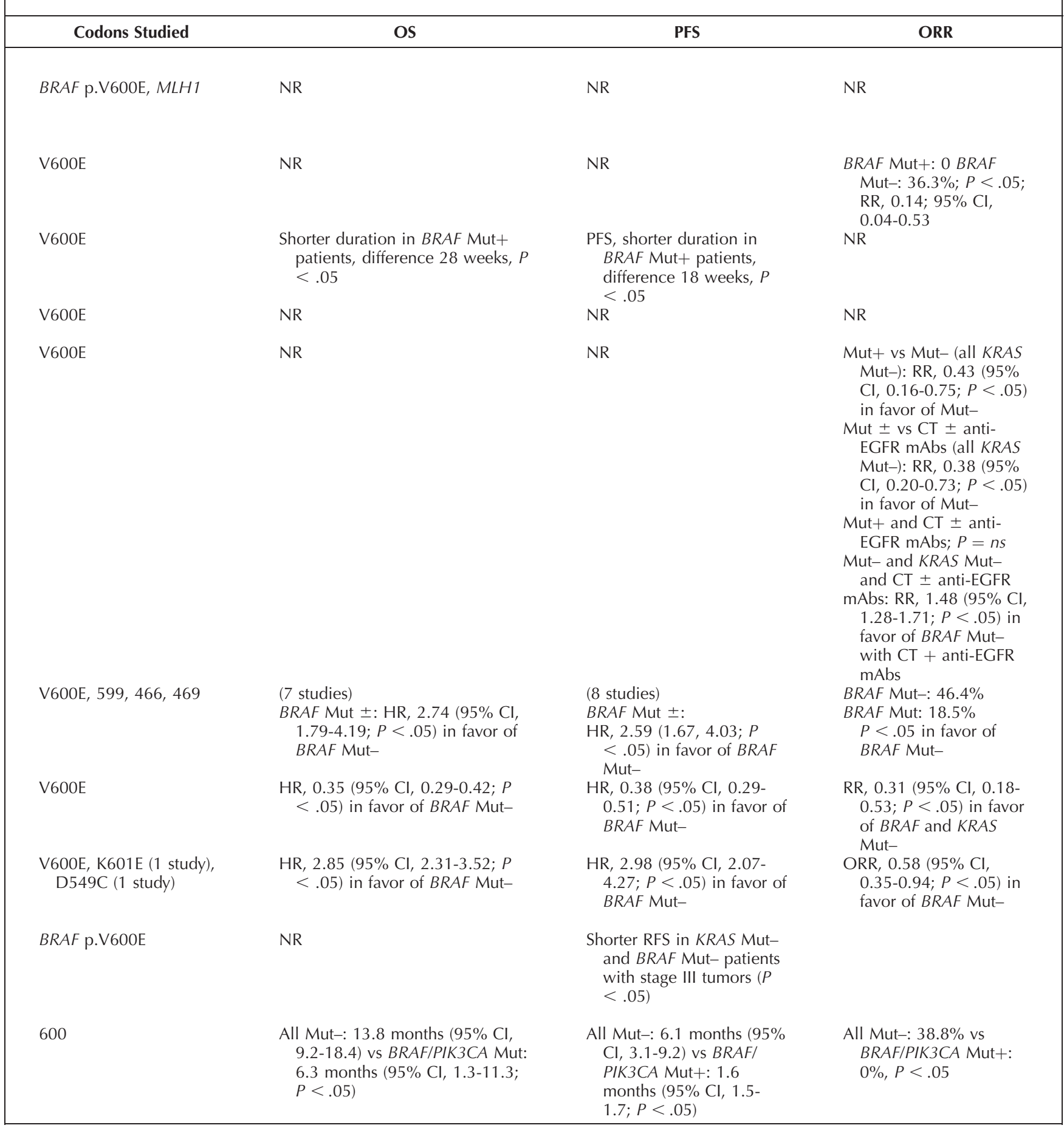

effective in this population. ${ }^{57-59}$ Data in support of molecular testing for BRAF c.1799 (p.V600) mutations in CRC continue to emerge from clinical trials. A recent publication of the PETACC-8 (oxaliplatin, fluorouracil, and leucovorin with or without cetuximab in patients with resected stage III colon cancer randomised phase 3) trial reported that trials in the adjuvant setting should consider mismatch repair, $B R A F$, and KRAS status for stratification, since BRAF p.V600 and KRAS mutations were associated with shorter DFS and OS in patients with microsatellite-stable colon cancer but not in those with tumors with MSI. ${ }^{60,61}$

This recommendation is supported by seven systematic reviews, ${ }^{20,47,48,50-53}$ three of which included meta-analysis. ${ }^{47,48,53}$ None of the systematic reviews reported the composition of their panel, so multidisciplinary panel representation could not be confirmed, and none reported patient representation on the panel. All but the systematic review reported by Baas et $\mathrm{al}^{20}$ reported examining important patient subgroups. All of the systematic reviews 


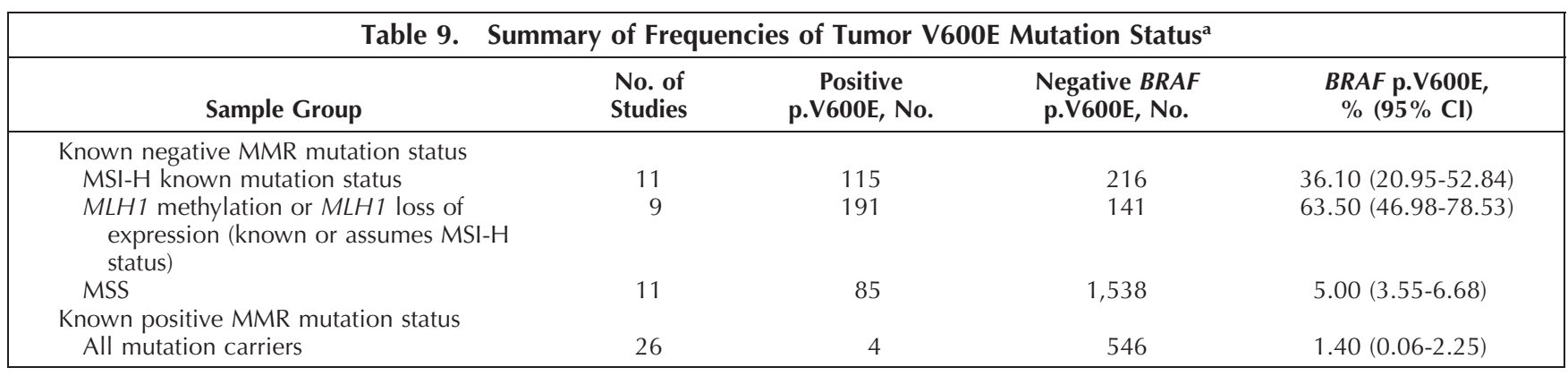

$B R A F$, proto-oncogene B-Raf/v-Raf murine sarcoma viral oncogene homolog $\mathrm{B} ; \mathrm{Cl}$, confidence interval; MMR, mismatch repair; $\mathrm{MSI}-\mathrm{H}$, microsatellite instability high; MLH1, mutL homolog 1; MSS, microsatellite stable.

a Adapted from Parsons et $\mathrm{a}^{52}$ by permission from BMJ Publishing Group Limited. ㄷ 2012.

reported well-described and reproducible methods. Three did not report how conflicts of interest were managed and reported on. ${ }^{47,51,53}$ Only two reported on a quality assessment of the included literature, ${ }^{48,50}$ and only one rated the strength of the evidence. ${ }^{50}$ None reported a plan for updating. While none of the systematic reviews reported industry funding, one study did not report any funding support. ${ }^{47}$ Overall, the risk of bias assessment for this body of evidence ranged from $\operatorname{low}^{48,50}$ to moderate, ${ }^{20,51,53}$ and none were found to have methodologic flaws that would raise concerns about their findings.

2b. Recommendation.-BRAF p.V600 mutational analysis should be performed in dMMR tumors with loss of MLH1 to evaluate for Lynch syndrome risk. Presence of a $B R A F$ mutation strongly favors a sporadic pathogenesis. The absence of BRAF mutation does not exclude risk of Lynch syndrome.

dMMR occurs via several mechanisms. In sporadic CRC, $\mathrm{dMMR}$ is most frequently caused by epigenetic silencing through CpG methylation primarily of MLH1, with few cases resulting from somatic mutation of one of the MMR genes. In Lynch syndrome CRC, the underlying mechanism is usually a germline mutation of one of the four (MLH1, MSH2, MSH6, and PMS2) mismatch repair genes and, in rare patients, a deletion involving EPCAM (epithelial cell adhesion molecule), a gene adjacent to $\mathrm{MSH}$, that leads to epigenetic inactivation of the MSH2 gene. dMMR occurs in $15 \%$ to $20 \%$ of all colorectal cancers, and of these, about three-fourths are due to MLH1 epigenetic silencing., ${ }^{5,62}$ dMMR underlies widespread mutations in the genome and MSI. BRAF p.V600 mutations rarely occur in patients with germline-based dMMR but have been reported in up to three-fourths of those with epigenetic MMR gene silencing (Table 8 and Table 9). Thus, testing for BRAF mutations serves as a means for distinguishing germline from epigenetic dMMR, particularly in those cases where the dMMR is the result of epigenetic silencing of MLH1. For tumors with a mutation in BRAF and dMMR, it may be concluded that the basis for their dMMR is less likely to be germline.,52,62 In contrast, tumors with $\mathrm{dMMR}$ in the absence of a BRAF mutation may have either germline or an epigenetic ( $M L H 1$ gene promoter hypermethylation) basis for the dMMR, and specific testing for MLH1 promoter hypermethylation may be used to further refine the risk of Lynch syndrome before initiating definitive genetic testing. Identification of those patients with germline-based dMMR has clear implications for the patient's family members.

3. Recommendation.-Clinicians should order mismatch repair status testing in patients with colorectal cancers for

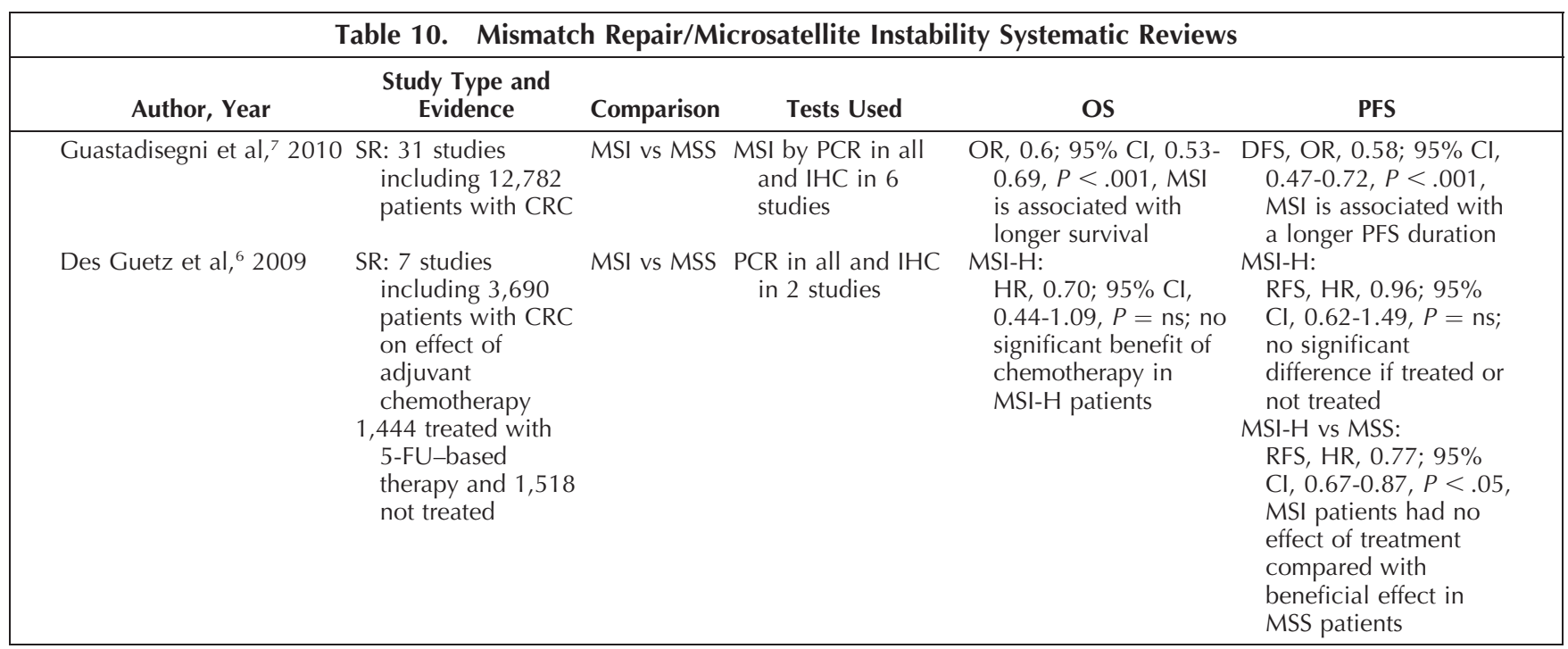

$\mathrm{Cl}$, confidence interval; CRC, colorectal cancer; DFS, disease-free survival; 5-FU, 5-fluorouracil; HR, hazard ratio; IHC, immunohistochemistry; MSI, microsatellite instability; MSI-H, microsatellite instability high; MSS, microsatellite stable; ns, nonsignificant; OR, odds ratio; OS, overall survival; PCR, polymerase chain reaction; PFS, progression-free survival; RFS, relapse-free survival; SR, systematic review. 
the identification of patients at high risk for Lynch syndrome and/or prognostic stratification.

The molecular pathology underlying most MSI tumors is somatically acquired $\mathrm{CpG}$ methylation of the promoter of the MLH1 gene. About three-fourths of colorectal cancers with MSI due to MLH1 promoter hypermethylation will have an acquired BRAF mutation as well. The reason for this is not understood. Less than one-third of individuals with dMMR/MSI colorectal tumors do not have underlying MLH1 promoter hypermethylation but rather have a germline mutation affecting any one of the four DNA MMR genes noted above. Individuals with germline mutations in the MMR genes are said to have Lynch syndrome, an autosomal dominant disorder that confers dramatically increased risks for colorectal and endometrial cancers and moderately increases risks for a variety of other tumors. ${ }^{63}$ Diagnosis of Lynch syndrome is important as active management of cancer risks has been demonstrated to benefit gene mutation carriers, ${ }^{5,64,65}$ and establishing a diagnosis creates opportunities for prevention among all at-risk relatives. Testing for dMMR can be performed by immunohistochemistry for the four MMR proteins (MLH1, MSH2, PMS2, and MSH6) or by MSI DNA-based testing, as discussed in detail in a report by Funkhouser et $\mathrm{al}^{66}$ (recommendation 11).

A systematic review of 31 studies $^{7}$ reporting survival on 12,782 patients whose tumors were characterized for MSI showed a favorable prognosis, as determined by both OS and DFS (Table 10), but this is dependent on stage. In addition, the presence of MSI in CRC was reported to be predictive for nonresponse to 5-fluorouracil-based adjuvant chemotherapy of early stage disease, ${ }^{6}$ although this has not been corroborated (Table 10). ${ }^{67}$ Emerging data indicate that MMR status may have predictive value in some settings, specifically in patients with advanced disease being considered for anti-programmed cell death protein-1 (PD-1)/ programmed cell death ligand protein-1 (PD-L1) immune checkpoint inhibitor therapy. ${ }^{68-70}$

This recommendation is supported by two systematic reviews that included 38 studies and 16,472 patients. ${ }^{6,7}$ Both of these systematic reviews included a well-described and reproducible methods section, and both reported on potential conflicts of interest. Only one, the systematic review reported by Guastadisegni et al, ${ }^{7}$ reported the source of funding, which was nonindustry. Due to deficits in the reporting, one of these systematic reviews was deemed to have a moderate risk of bias, ${ }^{6}$ and the other was deemed to have a low to moderate risk of bias ${ }^{7}$; however, neither of these were found to have any major methodologic flaws that would cause us to question their findings.

4. No Recommendation.-There is insufficient evidence to recommend BRAF c.1799 (p.V600) mutational status as a predictive molecular biomarker for response to anti-EGFR inhibitors.

As noted in recommendation $2 \mathrm{a}$, mutations in position p.V600 in BRAF are associated with poor prognosis, especially in patients with metastatic disease. Response rates to chemotherapy regimens, including regimens with cetuximab and panitumumab, are lower in patients harboring BRAF p.V600 mutations ${ }^{51,53,71}$ (Table 8). Similarly, the PFS and OS after treatment with EGFR monoclonal antibodies in combination with chemotherapy are lower in patients with BRAF p.V600 mutations. ${ }^{47,48}$ Many of these analyses used nonrandomized cohorts, thereby making evaluation of the potential predictive value of the BRAF
p.V600 mutation impossible to discern (Table 8). In addition, the poor prognosis and low mutation prevalence make evaluation of the relative benefit of EGFR inhibitors difficult to evaluate in individual randomized clinical trials.

Meta-analyses of randomized studies of EGFR monoclonal antibodies have been completed to address the question of the predictive role of BRAF p.V600 mutations. A metaanalysis of 463 patients with KRAS wild-type and BRAF p.V600 mutated tumors did not provide sufficient evidence to exclude a magnitude of benefits seen in KRAS/BRAF wildtype tumors. Nor was there sufficient evidence to identify a statistically significant benefit to this treatment. ${ }^{55}$ A second meta-analysis showed that EGFR monoclonal antibody treatment in patients whose tumors contain a BRAF p.V600 mutation was not associated with significant OS $(P$ $=.43)$, although there was a trend for better PFS $(P=.07){ }^{72}$ This suggests insufficient evidence to recommend the use of BRAF p.V600 as a predictive marker for benefit of anti-EGFR monoclonal antibodies. More data are required to definitively determine the predictive value of $B R A F$ mutations relative to anti-EGFR therapy.

This recommendation was supported by five systematic reviews ${ }^{47,48,51,53,71}$ (Table 8). None of these systematic reviews reported forming a multidisciplinary panel, and none reported including patient representatives in developing their research questions or interpreting their outcomes. All of the systematic reviews examined important patient subtypes, and all used well-described and reproducible methods. Only the systematic review by Yuan et $\mathrm{al}^{48}$ reported on any potential conflicts of interest, the article by $\mathrm{Mao}$ et $\mathrm{al}^{51}$ stated conflicts were not examined, and the other three did not report anything regarding conflicts. ${ }^{47,53,71}$ Only two, the systematic reviews reported by Yang et $\mathrm{al}^{71}$ and Yuan et $\mathrm{al}^{,{ }^{48}}$ rated the quality of the included evidence, although none of the studies reported on the strength of the evidence. None of the studies discussed any plans for future updating. Four reported nonindustry funding for their systematic reviews, ${ }^{48,51,53,71}$ and one did not report the source of funding, if any. ${ }^{47}$ Two of the systematic reviews were deemed to have a low risk of bias, ${ }^{48,71}$ one was deemed to have a low to moderate risk of bias, ${ }^{47}$ and two were deemed to have a moderate risk of bias. ${ }^{51,53}$ Overall, none of the systematic reviews were found to have methodologic flaws that would raise concerns about their findings.

5. No Recommendation.-There is insufficient evidence to recommend PIK3CA mutational analysis of colorectal carcinoma tissue for therapy selection outside of a clinical trial.

Note: Retrospective studies have suggested improved survival with postoperative aspirin use in patients whose colorectal carcinoma harbors a PIK3CA mutation.

Despite comprehensive RAS testing (recommendation 1), many patients still fail to respond to EGFR monoclonal antibody therapy. Additional biomarkers to guide patient selection for such therapy are desired.

PIK3CA mutations are observed in $10 \%$ to $18 \%$ of patients with CRC, primarily in exons 9 and 20, and lead to a constitutive activation of p100a enzymatic activity, leading to an increased PI3K activity and high oncogenic transformation ability. However, mutations of KRAS or NRAS and PIK3CA mutations can be detected alternatively and, in some cases, concurrently in a single CRC. ${ }^{3,8}$ PIK3CA mutations are positively correlated with KRAS exon 12 and 13 mutations. $^{3}$ Several meta-analyses and one individual patient data large pooled analysis have examined the 
Table 11. PIK3CA Clinical Practice Guidelines, Systematic Reviews, Meta-Analyses, Prospective Cohort Studies, and Retrospective Cohort Studies

\begin{tabular}{|c|c|c|c|}
\hline Author, Year & Study Type and Evidence & Comparison & Tests Used \\
\hline \multicolumn{4}{|c|}{ CPGs, systematic reviews, and meta-analyses on PIK3CA Mut+ vs Mut- $(\mathrm{n}=5)$} \\
\hline Wu et $\mathrm{al}^{, 33} 2013$ & $\begin{array}{l}\text { SR: } 8839 \text { patients with } \\
\text { mCRC who all received } \\
\text { anti-EGFR mAbs }\end{array}$ & Mut+ vs Mut- & $\begin{array}{l}\text { Sanger, allelic } \\
\text { discrimination, direct } \\
\text { sequencing, } \\
\text { pyrosequencing }\end{array}$ \\
\hline Mao et al, $^{33} 2012$ & $\begin{array}{l}\text { SR: } 13 \text { studies including } \\
\text { patients all KRAS Mut- } \\
\text { treated with anti-EGFR } \\
\text { mAbs }\end{array}$ & E20 Mut+ vs E20 Mut- & NR \\
\hline Lin et $a^{, 50} 2011$ & $\begin{array}{l}\text { SR: } 4 \text { studies } 1,030 \\
\text { patients with mCRC, all } \\
\text { KRAS Mut- subgroup } \\
\text { analysis, exons } 9 \text { and } 20\end{array}$ & Mut+ vs Mut- & NR \\
\hline Baas et al, $^{20} 2011$ & $\begin{array}{l}\text { SR: } 3 \text { studies including } 195 \\
\text { patients with mCRC }\end{array}$ & Mut+ vs Mut- & $\begin{array}{l}\text { Sequencing, } \\
\text { pyrosequencing }\end{array}$ \\
\hline \multicolumn{4}{|c|}{ Retrospective cohort studies $(\mathrm{n}=1)$} \\
\hline Bando et al, ${ }^{43} 2013$ & $\begin{array}{l}82 \text { samples from } 376 \\
\text { patients }\end{array}$ & $\begin{array}{l}\text { All Mut- vs BRAF Mut+ } \\
\text { and PIK3CA Mut+ }\end{array}$ & $\begin{array}{l}\text { Luminex xMAP vs DS } \\
\text { (concordance rate } \\
100 \%)\end{array}$ \\
\hline
\end{tabular}

AS-PCR, allele-specific polymerase chain reaction; $\mathrm{Cl}$, confidence interval; CPG, clinical practice guideline; DS, direct sequencing; EGFR, epidermal growth factor receptor; HR, hazard ratio; mAbs, monoclonal antibodies; mCRC, metastatic colorectal cancer; Mut-, mutation negative or wild type; Mut+, mutation positive; NR, not reported; ns, nonsignificant; ORR, objective response rate; OS, overall survival; PCR, polymerase chain reaction; PFS, progression-free survival; PIK3CA, phosphatidylinositol-4,5-bisphosphate 3-kinase catalytic subunit alpha; RR, response rate; RT-PCR, reverse transcription polymerase chain reaction; SR, systematic review; xMAP, multiplex assay.

prognostic role of PIK3CA in patients with stage IV CRC, both overall and in the KRAS nonmutated/wild-type population. These studies have generally indicated poorer response rate and PFS in patients with the PIK3CA mutation, a finding that appears to be driven primarily by patients with exon 20 mutation $^{3,33,50,71}$ (Table 11). These meta-analyses have included many of the same studies, as well as observed and acknowledged between-study heterogeneity, and all have concluded further prospective data are necessary. Contradictory recent studies have also been recently reported. ${ }^{74}$ None of the studies considered the independent role of PIK3CA in the context of comprehensive $R A S$ testing. De Roock et $\mathrm{al}^{3}$ estimated that comprehensive PIK3CA testing would increase response rate in the first-line setting by only $1 \%$. The prognostic impact of PIK3CA in stage I to III disease has been inconsistent. ${ }^{75-77}$

Multiple prospective observational studies have demonstrated an association between aspirin use and decreased CRC mortality. ${ }^{7-80}$ Data on aspirin as a treatment for CRC (postdiagnosis usage) are more limited and drawn only from observational studies. Domingo et $\mathrm{al}^{81}$ and Liao et $\mathrm{al}^{82}$ found a survival advantage for posttreatment aspirin users only in patients whose tumors exhibit PIK3CA mutations; however, a recent cohort study did not validate these observations. ${ }^{83}$ Multiple prospective studies are under way to address the potential benefit of adding aspirin or other nonsteroidal anti-inflammatory drugs to adjuvant therapy.

This recommendation is supported by two systematic reviews ${ }^{33,40}$ obtained from our systematic review. None reported the composition of a multidisciplinary panel, reported patient representation or study quality, rated strength of the evidence reviewed, or disclosed a plan for updating. However, both systematic reviews did include relevant patient subgroups and included methods that were well described and reproducible. In both systematic reviews, information about the potential conflicts of the panelists was reported, and funding was provided by nonindustry sources. Both were found to have a moderate risk of bias, but neither of the studies providing the evidence base for recommendation 5 were found to have methodologic flaws that would raise concerns about their findings.

At the present time, the retrospective data for the use of PIK3CA mutation to deny anti-EGFR antibody therapy in patients with stage IV CRC or as a selection factor for use of aspirin in stage I to III tumors are insufficient for clinical use outside of a clinical trial.

6. No Recommendation.-There is insufficient evidence to recommend PTEN analysis (expression by immunohistochemistry $[\mathrm{IHC}]$ or deletion by fluorescence in situ hybridization [FISH]) in colorectal carcinoma tissue for patients who are being considered for therapy selection outside of a clinical trial.

PTEN functions as a tumor suppressor gene, and loss of PTEN results in upregulation of the PI3K/AKT pathway. PTEN mutations occur in approximately $5 \%$ to $14 \%$ of colorectal cancers, ${ }^{4,84}$ and loss of PTEN expression can be observed in tumors with KRAS, BRAF, and PIK3CA mutations. 
Table 11. Extended

\begin{tabular}{|c|c|c|c|}
\hline Codons Studied & OS & PFS & ORR \\
\hline Exons 9, 20 & $\begin{array}{l}\mathrm{HR}, 1.28 ; 95 \% \mathrm{Cl}, 1.05- \\
1.56, P<.05 \text {, patients } \\
\text { with PIK3CA Mut+ had } \\
\text { shorter PFS }\end{array}$ & $\begin{array}{l}\text { PFS, HR, } 1.53 ; 95 \% \mathrm{Cl}, 1.28- \\
1.84, P<.05, \text { patients with } \\
\text { PIK3CA Mut+ had shorter PFS }\end{array}$ & NR \\
\hline Exon 20 & $\begin{array}{l}\mathrm{HR}, 3.29 ; 95 \% \mathrm{Cl}, 1.60- \\
\quad 6.74 ; P<.05\end{array}$ & $\begin{array}{l}\text { PFS, HR, 2.52; } 95 \% \mathrm{Cl}, 1.33- \\
4.78, P<.05, P I K 3 C A \text { exon } 20 \\
\text { mutations associated with } \\
\text { shorter PFS }\end{array}$ & $\begin{array}{l}\text { ORR\%: } \\
\text { Exon } 20 \text { Mut+: } 0 ; \\
\text { Exon } 20 \text { Mut-: } 37 \% \\
\quad \text { RR, } 0.25 ; 95 \% \mathrm{Cl}, 0.05-1.19, \\
\quad P=\text { ns (subset: } 377 \text { patients) }\end{array}$ \\
\hline Exons 9, 20 & $\begin{array}{l}P=\text { ns, no difference } \\
\text { between Mut }+ \text { and Mut- } \\
\text { patients } \\
\text { Exon } 20 \text { Mut }+ \text { predicts } \\
\text { poorer survival }\end{array}$ & $\begin{array}{l}P=\mathrm{ns}, \text { no difference between } \\
\text { Mut }+ \text { and Mut- patients } \\
\text { Exon } 20 \text { Mut }+ \text { predicts poorer } \\
\text { survival }\end{array}$ & NR \\
\hline Exons 9, 20 & NR & NR & NR \\
\hline $\begin{array}{l}\text { Exons } 7,8,9,18 \\
\quad 19,20\end{array}$ & $\begin{array}{l}\text { (6 studies) } \\
\mathrm{HR}, 1.43(95 \% \mathrm{Cl}, 1.02- \\
2.0 ; P<.05) \text { in favor of } \\
\text { Mut- }\end{array}$ & $\begin{array}{l}\text { (6 studies) } \\
\text { HR, } 1.91(95 \% \mathrm{Cl}, 0.78-4.68 ; P= \\
\text { ns) } \\
P<.05 \text { in favor of exon } 9 \\
\text { compared with exon } 20 \\
\text { mutations }\end{array}$ & $\begin{array}{l}\text { (6 studies) } \\
\text { RD: }-23 \%(-35 \%,-10 \% ; P< \\
.05) \text { in favor of exon } 9 \\
\text { compared with exon } 20 \\
\text { mutations }\end{array}$ \\
\hline Exon 9 & $\begin{array}{l}\text { All Mut-: } 13.8 \text { months } \\
\text { (95\% Cl, 9.2-18.4) vs } \\
\text { BRAF/PIK3CA Mut+: } 6.3 \\
\text { months }(95 \% \mathrm{Cl}, 1.3- \\
11.3 ; P<.05)\end{array}$ & $\begin{array}{l}\text { All Mut-: } 6.1 \text { months }(95 \% \mathrm{Cl} \text {, } \\
\text { 3.1-9.2) vs } B R A F / P I K 3 C A \text { Mut+: } \\
1.6 \text { months }(95 \% \mathrm{Cl}, 1.5-1.7 ; P \\
<.05)\end{array}$ & $\begin{array}{l}\text { All Mut-: } 38.8 \% \text { vs } B R A F / \\
\text { PIK3CA Mut+: } 0, P<.05\end{array}$ \\
\hline
\end{tabular}

Although there is evidence suggesting that PTEN is a critical factor in cancer development, the association between PTEN expression and predictive/prognostic value remains controversial, with several studies suggesting an association with poorer prognosis and others finding no association at all. Four systematic reviews were obtained that reported on loss of PTEN expression compared with normal PTEN expression and 31 primary studies, including a total of 2,545 patients ${ }^{20,50,85,86}$ (Supplemental Table 14). Tests used included IHC and FISH. Of the four studies that reported overall survival rates, ${ }^{20,50,85,86}$ three studies reported on pooled outcomes..$^{50,85,86}$ One study reported a significant difference in favor of normal PTEN expression, ${ }^{86}$ and the others reported no significant differences. ${ }^{20,50,85}$ For PFS, three studies pooled outcomes, ${ }^{50,85,86}$ two detected a significant difference in favor of normal PTEN expression, ${ }^{85,86}$ and one showed no significant difference. ${ }^{50}$ For ORR, two studies pooled outcomes, and both found loss of PTEN expression associated with a poorer response. ${ }^{85,86}$

Several studies have shown an association between PTEN loss and local recurrence, advanced TNM stage, lymph node metastasis, and a lower 5-year survival rate. ${ }^{87-90}$ However, several other studies have found no correlation between PTEN status and patient survival, tumor grade, TNM stage, lymphatic invasion, and liver metastasis. ${ }^{91-93}$ Regarding response to EGFR-targeted therapies, several studies have shown an association with PTEN loss and lack of response to cetuximab and panitumumab. ${ }^{94-97}$ However, other published studies failed to demonstrate a clear correlation between loss of PTEN expression and response to antiEGFR therapy. ${ }^{98-100}$ Given the significant discordance in results, the role of PTEN as a prognostic or predictive biomarker in CRC is still largely unknown, and research into the prognostic and predictive significance of PTEN is ongoing.

This recommendation is supported by 20 studies, ${ }^{4,20,50,84-100}$ four ${ }^{20,50,85,86}$ of which met the inclusion criteria for inclusion in our systematic review. All four of these were systematic reviews and included 42 studies and 3,412 patients. None of these systematic reviews reported using a multidisciplinary panel or reported including the patient perspective or a plan for future updating. Three ${ }^{50,85,86}$ reported on important patient subgroups. All four had well-described and reproducible methods sections. Three $20,50,86$ reported that potential conflicts of interest were examined. Only two $0^{50,86}$ rated the quality of the included evidence, and these same two were also the only two that rated the strength of the evidence. Only three $20,50,86$ reported on the source of any funding, but all three reported nonindustry funding. One was deemed to have a low risk of bias, ${ }^{50}$ one was deemed to have a low to moderate risk of bias, ${ }^{86}$ and two were deemed to have a moderate risk of bias. ${ }^{20,85}$ None of the studies were found to have any methodologic flaws that would bring doubt to their findings.

7. Expert Consensus Opinion.-Metastatic or recurrent colorectal carcinoma tissues are the preferred specimens for treatment predictive biomarker testing and should be used if such specimens are available and adequate. In their absence, primary tumor tissue is an acceptable alternative and should be used.

In clinical practice, one or more specimens of CRC from an individual patient may become available for molecular testing during the course of the disease. These specimens may include initial diagnostic biopsy or surgical resection specimens of the primary tumor and resection, biopsy, or cytologic specimens from metastatic and recurrent tumor. 


\begin{tabular}{|c|c|}
\hline \multicolumn{2}{|c|}{$\begin{array}{c}\text { Table 12. Concordance Rates Between Primary and } \\
\text { Metastatic Lesions }{ }^{\mathrm{a}}\end{array}$} \\
\hline Genes Tested (n) & Concordance Rate, $\%$ \\
\hline KRAS $(117)^{101}$ & 91.0 \\
\hline KRAS, NRAS, BRAF $(84)^{102}$ & 98.8 \\
\hline PIK3CA $(117)^{101}$ & 94.0 \\
\hline PIK3CA $(84)^{102}$ & 92.8 \\
\hline PTEN IHC $(117)^{101}$ & 66.0 \\
\hline
\end{tabular}

a Summary of two randomized clinical trials where comparison of mutation in KRAS, NRAS, BRAF, and PIK3CA was performed for paired primary tumor and metastatic lesions. Immunohistochemistry for PTEN was done in Cejas et al. ${ }^{101}$ In the study by Cejas et al, ${ }^{101}$ metastases were synchronous or metachronous. DNA was extracted from formalin-fixed, paraffin-embedded tissue, and mutational analysis was performed with a polymerase chain reaction-direct sequencing assay. KRAS mutations were detected in $42 \%$ of metastatic lesions and $39 \%$ of primary tumors. In the study by Vakiani et al, ${ }^{102}$ DNA was extracted from frozen tissue, and the iPLEX assay (Agena Bioscience, San Diego, CA) was used to examine the following mutations: KRAS $12,13,22,61,117$, and 146; NRAS 12, 13, and 61; BRAF 600; and PIK3CA 345, 420, 542, 545, 546, 1043, and 1047.

Discordance between primary and metastatic lesions may be attributed to a number of mechanisms, including tumor heterogeneity already present in the primary tumor, tumor evolution, where novel mutations are acquired, and, in some cases, the presence of separate primaries. The systematic literature review for the CRC guideline was done to identify studies that compared the mutational status of primary versus metastatic CRC.

An earlier systematic literature search that was conducted to include studies testing concordance of KRAS, BRAF, PIK3CA, and loss of PTEN expression in $\mathrm{CRC}^{20}$ reported the results of 21 studies, with an overall concordance rate of 93\% (range, 76\%-100\%) for KRAS, 93\% for BRAF status, a range of $89 \%$ to $94 \%$ for PIK3CA, and $68 \%$ for loss of PTEN. Table 12 shows the summary of two subsequent studies where KRAS, NRAS, BRAF, and PIK3CA mutation and PTEN expression were compared in paired primary versus metastatic tumor lesions. ${ }^{101,102}$ Overall concordance rates between primary and metastatic lesions were high with more than $90 \%$ concordance (Table 12). ${ }^{101,102}$ In the study by Lee et al, analysis of KRAS mutation in primary and recurrent tumors after radical resection showed 20.3\% discordance. ${ }^{103}$

This recommendation was supported by two retrospective cohort studies ${ }^{101,102}$ that were obtained in the systematic review. Both of these studies compared results within a single cohort. The study reported by Cejas et $\mathrm{al}^{101}$ reported at least partial industry funding, and the study reported by Vakiani et $\mathrm{al}^{102}$ did not report the source of funding, if any. The study by Cejas et $\mathrm{a}^{101}$ was deemed to have a low to moderate risk of bias, and the study by Vakiani et $\mathrm{al}^{102}$ was deemed to be low.

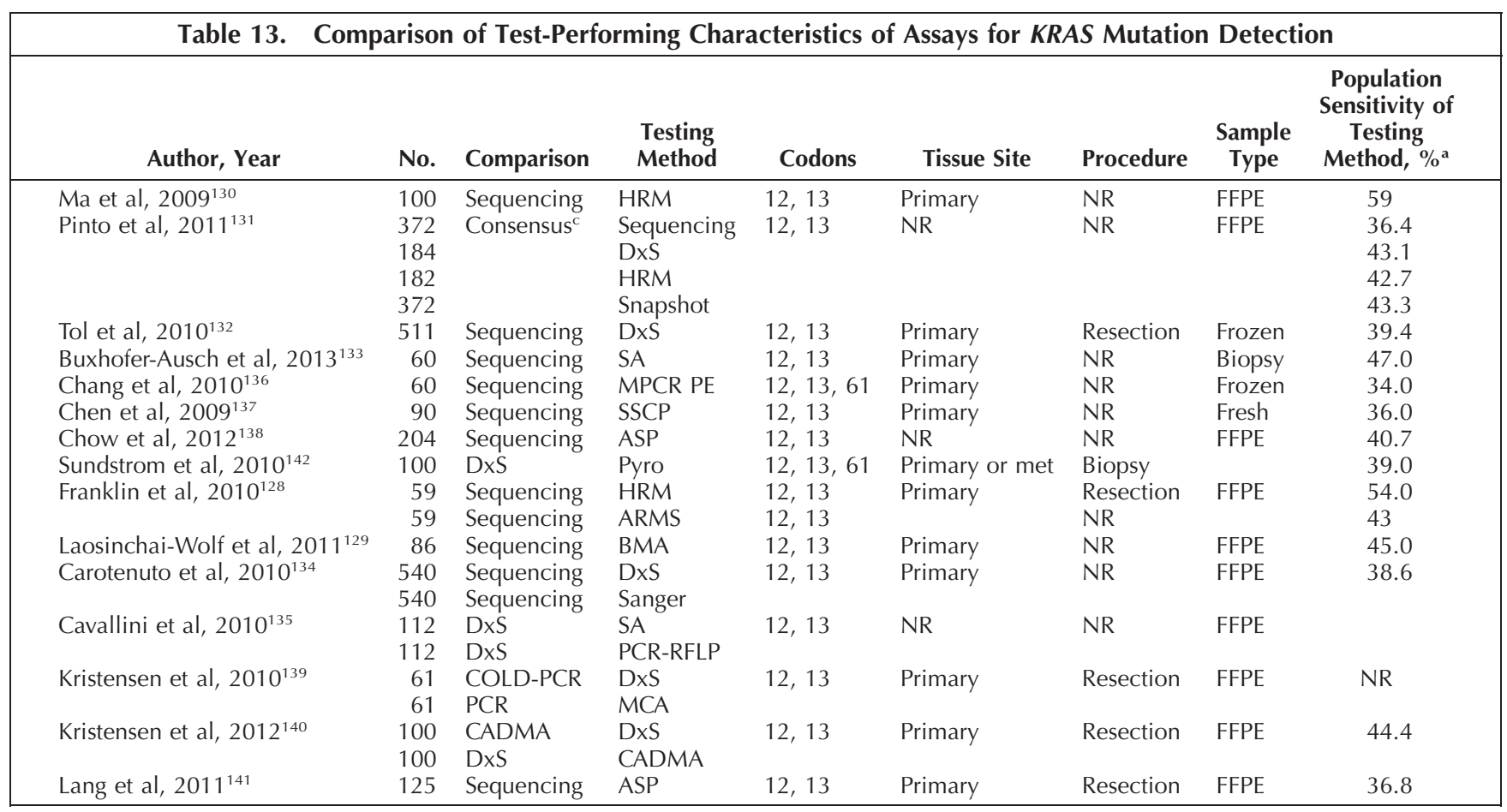

ARMS, amplification refractory mutation system; ASP, allele specific (nonquantitative); BMA, Luminex bead microarray; CADMA, competitive amplification of differentially melting amplicons; COLD-PCR, coamplification at lower denaturation temperature-PCR; DxS, QIAGEN method; FFPE, formalin fixed, paraffin embedded; HRM, high-resolution melting; M, missing; MCA, melting curve analysis; Met, metastatic; MPCR PE, multiplex polymerase chain reaction (KRAS, NRAS, HRAS) and primer extension; Mut, mutation; NPV, negative predictive value; NR, not reported; PCR-RFLP, polymerase chain reaction-restriction fragment length polymorphism; PCS, prospective cohort study; PPV, positive predictive value; Pyro, pyrosequencing; RCS, retrospective cohort study; SA, KRAS-BRAF strip assay; SSCP, single-strand conformation polymorphism.

a Population or clinical sensitivity of testing method (\%) of cases positive for KRAS mutation tested.

${ }^{\mathrm{b}}$ Four $(9.5 \%)$ of 42 samples negative for KRAS mutation by direct sequencing were positive for KRAS mutations by HRM analysis.

c Total of $84.4 \%$ of consensus mutation result.

d Detected one mutation in 23 Mut- alleles.

e Variable concordance for different tumor percentage in the sample.

${ }^{\mathrm{f}}$ The sensitivity was increased by 5 - to 100 -fold for melting temperature decreasing mutations when using COLD-PCR compared with standard PCR. Mutations, undetectable by the TheraScreen (QIAGEN, Valencia, CA) kit in clinical samples, were detected by COLD-PCR followed by HRM and verified by sequencing. Sequencing (PCR of fragment of interest followed by sequence analysis) described as direct sequencing. 
Overall, neither of these studies had any methodologic flaws that would raise concerns about the reported findings.

In summary, given that discordance of mutational status between primary and metastatic or recurrent CRC lesions may occur in a number of cases, metastatic or recurrent CRC tissues are the preferred specimens for treatment predictive biomarker testing. However, if these specimens are not available, primary tumor tissue is an acceptable alternative, given the overall high rates of concordance for the mutation status of EGFR pathway genes.

8. Expert Consensus Opinion.-Formalin-fixed, paraffin-embedded (FFPE) tissue is an acceptable specimen for molecular biomarker mutational testing in colorectal carcinoma. Use of other specimens (eg, cytology specimens) will require additional adequate validation, as would any changes in tissue-processing protocols.

The systematic review identified a number of studies, summarized in Table 13, where CRC KRAS mutational testing was performed using FFPE specimens as well as fresh or frozen specimens. Recommendation 17 highlights the importance of review of stained sections of tumor selected for testing by a pathologist to verify the tumor cell content population of the sample and demarcate regions for potential macrodissection or microdissection to enrich for cancer cells. Biopsy and resection specimens are similarly acceptable, as long as sufficient tumor cells are present (Table 13). Cytology specimens may be adequate for testing but will require proper validation. The use of FFPE cell blocks allows for the evaluation of tumor cell content and viability. ${ }^{104}$ Laboratories will need to establish the minimum tumor cell content for specimens based on the performance characteristics of their validated assay. ${ }^{105,126}$

Liquid biopsy tests use serum or plasma and may be used for monitoring tumor recurrence and emergence of treatment resistance. The noninvasive nature of this approach (monitoring through blood testing) offers great potential for clinical use. ${ }^{106}$ However, at the present time, the clinical application of liquid biopsy assays awaits robust validation and further studies to determine their clinical utility.

9. Strong Recommendation.-Laboratories must use validated colorectal carcinoma molecular biomarker testing methods with sufficient performance characteristics for the intended clinical use. Colorectal carcinoma molecular biomarker testing validation should follow accepted standards for clinical molecular diagnostics tests.

Clinical validation assesses the molecular biomarker testing method in light of clinical characteristics of the disease or marker being tested, to ensure the test is "fit for purpose." Elements of clinical validation include analytical sensitivity, analytical specificity, clinical sensitivity, and

\begin{tabular}{|c|c|c|c|c|c|c|c|}
\hline $\begin{array}{l}\text { Sensitivity } \\
\text { of Assay }\end{array}$ & $\begin{array}{c}\text { Analytical } \\
\text { Sensitivity, \% } \\
\text { (Mutant Allele } \\
\text { Fraction) }\end{array}$ & Specificity, \% & PPV, \% & NPV, \% & $\begin{array}{c}\text { Minimal } \\
\text { Tumor, \% }\end{array}$ & $\begin{array}{c}\text { Concordance } \\
\text { Between } \\
\text { Assays, \% }\end{array}$ & Study \\
\hline Increased $^{\mathrm{b}}(>100)$ & $5-10$ & 98 & NR & NR & 30 & 95 & PCS \\
\hline $84.4^{c}$ & $15-20$ & NR & NR & NR & $>50$ & NR & PCS \\
\hline 96 & 1 & NR & NR & NR & & NR & \\
\hline 98 & $3-10$ & NR & NR & NR & & NR & \\
\hline 99 & 5 & NR & NR & NR & & NR & \\
\hline 96.5 & 1 & 99.7 & 99.5 & 97.2 & $3-90$ & 95.30 & PCS \\
\hline 100 & 1 & 100 & NR & $\mathrm{NR}$ & At least 50 & 100 & PCS \\
\hline 100 & $N R^{d}$ & 100 & 100 & 100 & NR & 100 & PCS \\
\hline 100 & NR & 100 & 100 & 100 & NR & 100 & PCS \\
\hline 100 & $1.25-2.5$ & 100 & 100 & 100 & NR & NR & PCS \\
\hline 91 & $1.25-2.5 ; 1.25$ & NR & NR & NR & NR & NR & PCS \\
\hline 100 & 1 & 87 & 81 & 100 & $1-90$ & NR & RCS \\
\hline 100 & 5 & 71 & 66 & 100 & $1-90$ & 93 & RCS \\
\hline 100 & 1 & 100 & 100 & 100 & NR & NR or $M$ & RCS \\
\hline 95.8 & 1 & 100 & 100 & 97.3 & $<30$ vs $>70$ & Variable $^{\mathrm{e}}$ & RCS \\
\hline 98.6 & NR & 100 & 100 & 99.1 & NR & NR & RCS \\
\hline $92.5-100$ & NR & 100 & NR & $\mathrm{NR}$ & 70 & NR & RCS \\
\hline $92.5-100$ & NR & 100 & NR & NR & NR & NR & RCS \\
\hline 93 & $0.1-5$ & 100 & NR & NR & NR & $\mathrm{f}$ & RCS \\
\hline 97 & $5-10$ & 100 & NR & NR & NR & & RCS \\
\hline 98 & 0.50 & 98 & NR & NR & NR & 95.9 & RCS \\
\hline 99 & NR & 100 & NR & NR & NR & NR & RCS \\
\hline $95.7^{\mathrm{e}}$ & 1 & NR & NR & NR & $>50$ & NR & RCS \\
\hline
\end{tabular}


clinical specificity. Data for clinical validation can be obtained from studies performed by the laboratory, studies reported in peer-reviewed literature, or other reliable sources. CLIA requires clinical laboratories to have a qualified laboratory director who is responsible for ensuring that the laboratory provides quality laboratory services for all aspects of test performance. ${ }^{107}$ Rigorous validation should be performed to ensure all molecular marker testing methods, such as those used for colorectal carcinoma, are ready for implementation in the clinical laboratory. To reach that goal, each step of the testing process must be carefully evaluated and documented. Excellent and comprehensive documents have been published on this topic, and a detailed review is provided under recommendation 10 . Our systematic review of the available literature provided information regarding the performance characteristics of molecular marker testing methods of colorectal carcinoma in clinical use for RAS mutational testing (Table 13). Most studies reported the performing characteristic of assays that detected KRAS exon 2 mutations, as detailed in Table 13. Direct sequencing of genomic DNA, even after polymerase chain reaction (PCR) amplification of the fragment of interest, has low analytical sensitivity requiring a mutant allele frequency of about $20 \%$ for mutation detection. A number of more sensitive assays have been developed for RAS testing, including those listed in Table 13.

Sanger sequencing was used as the most common baseline assay for comparison against other molecular detection methods for KRAS mutations. Testing methods vary widely, including direct Sanger sequencing, amplification refractory mutation system, real-time PCR-highresolution melting (HRM) assays, allele-specific PCR, Luminex (Austin, Texas) bead microarray, PCR restriction fragment length polymorphism strip assays, pyrosequencing, and, more recently, NGS. Population or clinical sensitivity of the testing methods for KRAS mutations as shown in Table 13 ranged between 36\% and 59\%. Assay sensitivity ranged from $84.4 \%$ to $100 \%$, with Sanger sequencing on the lower end of the range. Analytical sensitivity, defined as the lowest detectable mutant allele fraction, was between $0.5 \%$ and $20 \%$ across all testing methods, with most methods performing between $1 \%$ and $5 \%$ mutant allele fraction. Specificity was between $98 \%$ and $100 \%$ for most assays, with two studies demonstrating lower specificity. Positive predictive value percentages varied between $66 \%$ and $100 \%$, with most studies reporting between $99 \%$ and $100 \%$. Negative predictive value percentages were between $97 \%$ and $100 \%$. Minimal tumor percentages reported varied widely between studies. Concordance between assays was between $93 \%$ and 100\%, with some variability noted in two retrospective cohort studies. The available evidence from assays to detect KRAS mutations supports the use of a number of alternative assays, as long as their performing characteristics, adjusted for sample type and percent tumor purity, meet the clinical sensitivity with acceptable specificity. Recently, NGS has been used in a number of studies and in laboratory practice for solid tumor mutational analysis. ${ }^{108}$ NGS has shown to meet the sensitivity of detection used in CRC clinical trials (detecting at least 5\% mutant alleles), permitting simultaneous testing of hundreds of mutations, and is becoming widely used. Testing for mutations in multiple genes or gene loci with multiplex assays such as NGS and other methods should be done on patients at the time of metastases to obtain comprehensive genomic information and identify mutations beyond $R A S / B R A F$ status that might be able to be targeted if conventional therapies become ineffective.

10. Strong Recommendation.--Performance of molecular biomarker testing for colorectal carcinoma must be validated in accordance with best laboratory practices.

Proper validation of CRC biomarker testing is important to ensure appropriate patient care. If validation is inadequate, this can lead to erroneous results and improper diagnosis, prognosis, and/or therapeutic intervention. For example, with regard to RAS testing, a false-positive result would lead to an improper withholding of therapy, whereas a false-negative result would lead to distribution of an ineffective therapy, resulting in increased costs and unnecessary side effects. As molecular oncology testing grows more complex with NGS, thorough and proper validation of preanalytical (specimen type and processing), analytical (assay performance), and postanalytical (bioinformatics, annotation, and reporting) steps is imperative. ${ }^{109,110}$

The design of a validation study somewhat depends on the analyte (gene), mutations, or molecular alterations assessed and chosen platform and technology. However, assay validation should be done using best laboratory practices in accordance with CLIA (42 CFR 493.1253(b)(2), also known as Title 42 Chapter IV Subchapter G Part 493 Subpart K§493.1253) ${ }^{111}$ as applicable to the assay type. Laboratories should comply with CLIA and their individual accrediting agency (eg, CAP, New York State) to fulfill requirements for validation. ${ }^{111,112}$ Additional resources for establishing clinical molecular testing are available to assist laboratories. ${ }^{113}$ For the US Food and Drug Administration (FDA)-cleared/approved assays (without any modification), verification of test specifications, including accuracy, precision, reportable range, and reference range, only needs to be done. ${ }^{114}$ For nonwaived, non-FDA-approved assays (laboratory-developed procedures or LDPs), validation must be performed. Validation design must include the required elements of analytical accuracy (specificity and sensitivity), precision, and analytical sensitivity (limit of detection) and interfering substances and reportable range as applicable. Clinical sensitivity and specificity, as well as positive and negative predictive value, should be considered additions.

Additional considerations should include specimen processing (including microdissection or macrodissection, histologic processing, and fixation times) and reagent stability and storage. Proper controls should be introduced and used to assess as many of the potential mutations detected by the assay and to verify the limit of detection identified in the validation. With high-throughput (NGS) sequencing, assessing all possible mutations through control material and specimens is impossible, and continuing validation may need to occur. If NGS is used, bioinformatics pipelines should be properly validated using multiple types of mutations (single-nucleotide variants and insertions/deletions). Finally, reporting should be carefully considered during the validation process. Resources to assist laboratories with solid tumor molecular testing have also been made available through the CLSI. ${ }^{115}$

\section{Preanalytical Variables}

Histologic or preanalytical processing should be considered and representative processes should be included in the validation set. Specific specimen types should also be properly validated. Most tissue used in CRC biomarker testing is derived from FFPE tissue. Formalin fixation results in fragmentation of DNA as a result of histone protein 
fixation to the DNA. Therefore, most assays for FFPE tissue are designed to amplify products less than 200 base pairs. Length of formalin fixation and age of blocks may also be factors to consider in validation of FFPE tissues. Other tissue sources should also be separately validated if offered as clinical tests, especially cytology-based specimens. Various cytology fixative preparations should be validated as used by the laboratory. If cell-free assays are considered, these should be validated as a separate source. Finally, testing should be limited to invasive carcinoma with exclusion of adenomatous tissue and benign background tissue cellular components (eg, normal mucosa, muscularis, inflammation) as much as possible.

\section{Analytical Variables}

Careful specimen selection should be undertaken to cover as many of the potential detected mutations and expected specimen types as possible to ensure analytical accuracy. A gold-standard method (dideoxy sequencing or other validated test method) and/or interlaboratory comparison should be used to verify accuracy of the assay. For example, the CAP Laboratory Accreditation Program COM.40350 indicates that at least 20 specimens (including positive, lowpositive, and negative specimens) should be included for qualitative and quantitative assays. ${ }^{112}$ More specimens may be required. If it is a single-gene assay, the design should include as many of the mutations covered by the assay as possible. If it is a real-time-based allele-specific assay, all mutations for which a primer probe reaction is built should be analyzed as reasonably as possible. If it is a pyrosequencing-based assay, similarly, all of the possible common mutations for which targeted therapies are indicated should be tested. Multigene assays based on NGS or other technology (such as SNaPshot [ThermoFisher Scientific, Waltham, MA]) require an increased number of specimens to test as many of the hotspot regions as possible in all genes included in the assay. With such assays, not all possible mutations can be validated. It is recommended that an ongoing validation occur after initial validation, with verification of novel mutations by either dideoxy sequencing or real-time PCR, depending on the laboratory capability and limit of detection. Depending on the technology employed, important parameters (eg, variant allele frequency, cyclic threshold values, allele coverage) should be monitored for interrun and intrarun precision.

CRC specimens can vary from large primary resection blocks with plenty of tumor cells to small primary tumor or metastatic CRC liver biopsy specimens to rectal specimens, after neoadjuvant therapy with minimal tumor percentage. Many of these tests are ordered for metastatic disease, for which only a small needle core biopsy specimen or cytologic sampling is available. Presently, tissue volume and accessibility are decreasing while ancillary testing (IHC and molecular studies) is increasing. The ability of an assay to be highly analytically sensitive is important if a laboratory is to test specimens with low tumor burden. It is recommended that an assay be able to identify a mutation in a specimen that has at minimum 20\% tumor cells (mutant allele frequency of $10 \%$ assuming heterozygosity). With NGS and highly sensitive PCR technologies, mutations should be identifiable in specimens with as little as 10\% tumor (mutant allele frequency of 5\% assuming heterozygosity and diploidy). Lower analytically sensitive assays, such as dideoxy sequencing, can be used, but it is recommended that PCR enrichment strategies (eg, coamplification at lower denaturation temperature-PCR) be used to increase the analytical sensitivity of the test and require less tumor percentage. A proper validation study should use cell line DNA (preferably FFPE treated) or reference control material manufactured by good manufacturing processes to assess limit of detection for as many mutations as possible. Importantly, the limit of detection may differ for mutations of varying types (small indels versus point mutations).

\section{Postanalytical Variables}

Postanalysis is as important to consider in validation as preanalytical and analytical variables. For single-gene assays, the software used in analysis should be validated, with verification of updates. If NGS is used, the bioinformatics pipeline should be thoroughly and rigorously validated, include potential problematic mutations (eg, large indels), and be verified or revalidated for new upgrades as applicable to the change. Any analysis should be performed on validation specimens as it would be for clinical specimens.

Reporting format should also be considered and decided during validation. Interpretation comments for inclusion in the patient report to ensure that the reports are correctly understood should be developed during the validation process. ${ }^{112}$ Human Genome Organisation (HUGO)-based nomenclature should be used for reports and a designated National Center for Biotechnology Information (NCBI) transcript number (NM_\#\#) should be used within the validation and report. ${ }^{116}$ For multigene panels based on NGS, reporting protocols and any used software should be included in the validation procedure. Databases and annotation guidelines should be discussed and included in the validation as one prepares to report variants based on NGS data. In addition, decisions should be made during the validation process as to whether normal tissue will be tested to assist in variant interpretation with NGS.

In conclusion, validation of assays used in CRC molecular testing is extremely important for accuracy of reporting and proper patient care. There are several documents (eg, CLIA, CAP, and CLSI) $)^{111-113,115}$ available to assist in proper validation, which should be consulted to validate according to best laboratory practices.

11. Strong Recommendation.-Laboratories must validate the performance of IHC testing for colorectal carcinoma molecular biomarkers (currently IHC testing for MLH1, MSH2, MSH6, and PMS2) in accordance with best laboratory practices.

Four proteins (MLH1, MSH2, MSH6, and PMS2) are currently considered important in the normal biochemistry of DNA MMR. ${ }^{117-119}$ As detailed in recommendation 2b, altered DNA mismatch repair proteins due to mutation or epigenetic silencing result in interference with normal MMR protein heterodimerization and loss of normal repair of mispaired bases and short insertions/deletions, resulting in MSI, 119,120 overall categorized as dMMR. Loss of MMR function usually correlates with loss of protein expression, such that immunohistochemical testing for MMR proteins is optimized to detect loss of MMR protein expression in tumor cell nuclei. Each of these four proteins can be detected in paraffin sections using commercially available primary and secondary antibodies, standardized antigen retrieval, and 3,3"-diaminobenzidine chromogen detection. Development of anti-MMR protein antibody staining protocols follows a standard approach that involves (1) demonstration of absent background noise with secondary 
antibody alone and (2) empirical optimization of the signalto-noise ratio by testing different antibody concentrations, antigen retrieval buffers, and reaction conditions, taking advantage of internal control cells, including lymphocytes, stromal cells, and other nonneoplastic nuclei.

Validation of the final staining protocol is required prior to implementation for clinical use. Peer-reviewed literaturebased guidelines for validation and revalidation of immunohistochemical tests have been defined as 14 recommendations and expert consensus opinions. ${ }^{121}$ Concordance with internal or external known comparator tests is required to exceed $90 \%$. Proficiency testing is a good approach to confirm interlaboratory test reproducibility. Test result concordance across laboratories implies accuracy of participant laboratory diagnosis.

Once the protocol is defined and validated for a given primary antibody clone and antigen retrieval conditions, a known positive external control (eg, tonsil) is routinely run in parallel with each unknown. This demonstrates that the MMR protein was detectable on that staining run and allows trust in a loss of expression result in the unknown specimen. Each of the four MMR proteins is expressed in nonneoplastic tissue, in most lymphocytes, and overexpressed in germinal centers, such that most colon block sections will also have positive internal control staining.

Overall, validated immunohistochemical detection of MMR proteins is a trustworthy method for identification of loss of expression of individual MMR proteins in paraffin sections of CRC. In most CRCs with high-level microsatellite instability (MSI-H), the loss of DNA MMR protein expression in tumor cell nuclei by immunohistochemical detection is uniform throughout the tumor. ${ }^{122,123}$ Rare cases of MSI tumors have been reported to show heterogeneous staining. ${ }^{124}$ Loss of MMR protein expression usually correlates with MSI, particularly for MSI-H tumors, and is indicative of $\mathrm{dMMR}$. If MSH2 or MLH1 shows loss of expression due to loss of function, then their heterodimer partners (MSH6 and PMS2, respectively) will also not be expressed. In contrast, inactivation of MSH6 or PMS2 results in loss of expression of the individual MMR protein MSH6 or PMS2, respectively.

Although loss of MMR protein immunoreactivity is generally detected in $\mathrm{AMMR} C \mathrm{C} C$, normal immunoreactivity can be seen in up to $10 \%$ of dMMR cases $^{125}$; therefore, MSI DNA testing may be performed either stepwise or as a concurrent test.

12. Expert Consensus Opinion.-Laboratories must provide clinically appropriate turnaround times and optimal utilization of tissue specimens by using appropriate techniques (eg, multiplexed assays) for clinically relevant molecular and immunohistochemical biomarkers of CRC.

Expediency in reporting of biomarker results for colorectal tumors is dictated primarily by two factors: need for patient management decisions and, more generally, patient anxiety. Consequently, results of such evaluations should be available within a timeframe for the involved clinician to relay this information to the patient. This need is compounded by the patient's need to receive a complete understanding of his or her diagnosis and treatment plans going forward. A reasonable benchmark is that nonacute biomarker results be available to the treating physician within 10 working days of receipt in the molecular diagnostics laboratory. This turnaround time has been recommended in other guidelines for molecular tumor testing. ${ }^{105,126,127}$ Ideally, the transitional time between test ordering, tissue block selection, block retrieval, and shipment to the performing laboratory should be included in the 10-day timeframe. Consequently, laboratories should make every effort to minimize delays in securing appropriate tissue blocks for testing. Testing laboratories should make every effort to minimize processing time and return of results.

The availability of tumor tissue for biomarker evaluation is generally not limiting in most cases of resected CRC. Occasionally, following neoadjuvant therapy, the amount of residual tumor in resection specimens can be very small and focal. Similarly, the amount of tumor tissue obtained by biopsy or fine-needle aspiration procedures from primary or metastatic foci can be very small and challenging to test for the desired biomarkers. In such circumstances, available tissue blocks should be sectioned judiciously, reserving sufficient sections for testing by molecular methods or immunohistochemical techniques, as deemed appropriate to secure as accurate and informative an evaluation as possible.

Test turnaround times for RAS testing in instances of advanced stage tumors are dictated by the need to select and initiate appropriate chemotherapy options. Ideally, such information should be available either at the time of postoperative oncology evaluation, where decisions regarding therapeutic options are entertained, or at the tumor boards where patient treatment options are discussed. In some institutions, these discussions may occur in the week following surgery or biopsy and probably no later than in the second week following tissue diagnosis and staging. Here, too, a timeframe of no more than 10 days would seem an appropriate benchmark for biomarker result availability.

In exceptional circumstances, even shorter test turnaround times may be appropriate. Occasional patients have histories sufficiently suggestive of Lynch syndrome that prompt consideration and discussion regarding extent of surgery (ie, complete colectomy or prophylactic hysterectomy in select affected patients). Efforts should be made in such circumstances to obtain appropriate test results as rapidly as possible to allow for informed decision making. MMR immunohistochemistry can be performed and reported with a turnaround time of 48 hours or less, and in the appropriate clinical context, a result of preserved expression of MMR proteins would argue against Lynch syndrome. Conversely, any loss of MMR protein expression will need to be integrated with additional clinical information, family history, and further testing such as BRAF mutation, MLH1 methylation testing, and potential germline genetic testing. Furthermore, DNA MMR status, performed by MMR immunohistochemistry or by MSI DNA tests, as a good prognostic biomarker for CRC overall, should be available within the recommended 10 working day turnaround time for test results.

13. Expert Consensus Opinion.-Molecular and IHC biomarker testing in colorectal carcinoma should be initiated in a timely fashion based on the clinical scenario and in accordance with institutionally accepted practices.

Note: Test ordering can occur on a case-by-case basis or by policies established by the medical staff.

Molecular and IHC biomarker testing is increasingly being used in patient management. Prognostic biomarkers are being used for early stage disease to guide decisions on the use of adjuvant chemotherapy. Such discussions require the availability of tests in a timely manner, and delays in initiation of therapy have been associated with worse outcomes. ${ }^{127}$ Predictive biomarkers, such as those for EGFR 
monoclonal antibody therapy, should be initiated in a timely fashion to guide chemotherapy options and long-term treatment planning. Institutional policies and practices that encourage the rapid initiation of appropriate molecular and IHC marker testing should be encouraged. Such policies may include reflexive ordering of molecular and IHC markers as guided by the clinical scenario and incorporation of testing initiation by multiple members of the multidisciplinary team, as noted in recommendation 15.

14. Expert Consensus Opinion.-Laboratories should establish policies to ensure efficient allocation and utilization of tissue for molecular testing, particularly in small specimens.

The number of molecular and immunohistochemical tests becoming available that have a direct benefit to patient care will continue to increase. Most of these tests are performed on FFPE specimens, the most common preservation technique, including pretreatment and posttreatment biopsies and resections (Table 13). Tissues from patients with cancer should be processed according to established laboratory protocols, which include quality controls of preservation materials, tissue dissection, time to fixation, fixation time, and processing.

Laboratory protocols need to include procedures for handling small samples such as endoscopic or core biopsy specimens and fine-needle aspirate samples of metastatic lesions (eg, from liver or lung). Limiting the number of tissue fragments per individual cassette is encouraged. Established protocols may allow upfront ordering of required tissue sections (eg, extra unstained slides), which limit tissue wasting and improve turnaround time of final results. Immunohistochemistry studies, if needed to diagnose metastatic CRC, should be limited in scope and standardized to preserve tissues.

It is imperative to identify suspected metastatic CRC specimens at specimen accessioning to limit unneeded ancillary tests, such as liver biopsy special stains. Recognition of previous CRC diagnoses from the patient clinical history should limit the need for immunohistochemistry profiles in many cases. Established laboratory procedures to identify patients undergoing cancer biopsy or fine-needle aspiration specifically for predictive molecular biomarker assessments need to be in place.

Laboratories must maintain appropriate cataloguing and storage of tissue specimens and diagnostic slides to allow for retrospective timely testing of cancer samples.

This recommendation is supported by 15 studies, ${ }^{128-142}$ comprising eight prospective cohort studies $130-133,136-138,142$ and seven retrospective cohort studies. ${ }^{128,129,134,135,139-141}$

For the eight prospective cohort studies, ${ }^{130-133,136-138,142}$ all reported balance between the treatment and assessment groups, as all but one ${ }^{132}$ used a single cohort design allowing for within-group comparisons. Only this single study, reported by Tol et $\mathrm{al}_{1}{ }^{132}$ would have required making adjustments for imbalances between the treatment and assessment groups, but none were needed. Five studies $^{130,133,136-138}$ reported nonindustry funding, one ${ }^{132}$ reported at least partial industry funding, one $\mathrm{e}^{142}$ reported industry funding, and one ${ }^{131}$ did not disclose the source of funding, if any. Seven $130,131,133,136-138,142$ were deemed to have a low risk of bias, and one ${ }^{132}$ was deemed to have a low to moderate risk of bias.

For the seven retrospective cohort studies, ${ }^{128,129,134,135,139-141}$ all used a single cohort design allowing for within-group comparisons. Four reported nonindustry funding, ${ }^{134,135,139,140}$ one reported industry funding, ${ }^{129}$ and two did not disclose the source of funding, if any. ${ }^{128,141} \mathrm{Six}$ were deemed to have a low risk of bias, ${ }^{128,134,135,139-141}$ and one was deemed to have a moderate risk of bias. ${ }^{129}$

All of the evidence that supported this recommendation was assessed, and none had methodologic flaws that would raise concerns about their findings.

15. Expert Consensus Opinion.-Members of the patient's medical team, including pathologists, may initiate colorectal carcinoma molecular biomarker test orders in accordance with institutionally accepted practices.

For patients with CRC, timely diagnosis or therapeutic initiation is critical, and molecular testing that is to be considered should be ordered as efficiently as possible in accordance with institutional practices and guidelines. MSI testing is often ordered at the time of diagnosis to identify patients with Lynch syndrome, direct adjuvant chemotherapy, or determine prognosis. Many institutions employ algorithms to ensure that all colorectal cancers are evaluated for MMR deficiency, and these are often initiated by pathologists when the diagnosis occurs after joint general process approval by pathologists, oncologists, and other members of the patient medical team. Molecular testing that is performed to direct targeted therapy (eg, RAS) may be ordered at a later date than the primary diagnosis, at metastatic presentation, for example, and so institutions may differ as to whether one should order such testing upfront on the primary diagnostic biopsy or resection specimen or wait until metastatic disease arises requiring targeted therapy. Often oncologists order predictive molecular assays since they are used to direct therapy, but this should not necessarily be limited to oncologists, as pathologists serve as important stewards of the tissue and make the tumor diagnosis. There are also issues to consider, including logistical issues, cost-effectiveness, patient access to molecular testing in rural or underserved areas, and even heterogeneity considerations between primary and metastatic tumor. Since each institution differs in patient population, facilities, departmental organization, regulatory and reimbursement climates, and practitioner preference, whether to submit testing at initial diagnosis of a primary lesion or when a metastatic lesion arises should be discussed collaboratively between oncologists, pathologists, and medical executive or hospital committees as applicable.

"Reflex" testing, a testing policy that does not require a separate clinician order for each case, is appropriate if agreed upon by the CRC care team as an institutionally approved standing order and may help to ensure expedited and consistent routing of specimens for molecular testing. However, some patients may not be candidates for targeted therapy for clinical reasons, and good communication between the clinical care team and the testing laboratory is needed to ensure testing is performed for patients whose management will be affected by the test result. Specifically, testing is not necessary for patients with stage IV disease who are being considered for palliative or hospice care only. Similarly, in settings in which reflex testing is the practice, a mechanism should be provided for the clinical care team to communicate to the pathologist examining a small biopsy or cytology sample when a more suitable diagnostic specimen (eg, a resection) is expected to be obtained, and the molecular testing should be deferred to the subsequent, more generous sample. All reflex testing should be approved institutionally by the hospital or institution's medical executive committee as local policies dictate. 
16. Expert Consensus Opinion.-Laboratories that require send out of tests for treatment predictive biomarkers should process and send colorectal carcinoma specimens to reference molecular laboratories in a timely manner.

Note: It is suggested that a benchmark of $90 \%$ of specimens should be sent out within 3 working days.

It is critical to provide the results of molecular tests in a timely fashion to start the most appropriate cancer treatment option for each patient. Delays in initiation of therapy have been associated with worse outcomes. ${ }^{127}$ To date, laboratories have had limited guidance on the recommended timing or turnaround time of molecular test results, and studies addressing the impact of specific turnaround times have not been conducted. Therefore, the panel reached an expert consensus opinion, based on each panel member's practical experience in the laboratory and clinical setting.

For laboratories that do not perform molecular testing and/or biomarker immunohistochemistry for CRC therapy selection, the consensus opinion was that send out of specimens should occur within 3 working days, starting from the day the test order was received in the laboratory, provided the specimens (eg, biopsy or resection specimens) are received at the same time of the test order or specimens are already in the laboratory (eg, archived paraffin blocks). The underlying rationale stems from the usual workflow for tissue processing. In practice, the longest process would be the processing of large surgical specimens, such as colectomies. A possible approach is to obtain a designated molecular tissue block at the time of specimen grossing, and molecular protocols for obtaining tissue sections may be used to have the necessary sections for test send-out in a timely fashion by the third working day for most cases. Another scenario may be the retrieval of archived tissue paraffin blocks that may be stored outside of the laboratory location. In this case, a protocol for block retrieval for molecular testing may be operationalized to streamline the process and reach the desired turnaround time for send-out. This turnaround time of 3 working days was also recommended for RAS testing of colorectal carcinoma in the guidance document from the Association of Clinical Pathologists Molecular Pathology and Diagnostics Group in the United Kingdom. ${ }^{126}$

Laboratories should develop written policies as part of their quality assurance program to monitor turnaround times for all cancer therapeutic and prognostic biomarkers.

17. Expert Consensus Opinion.-Pathologists must evaluate candidate specimens for biomarker testing to ensure specimen adequacy, taking into account tissue quality, quantity, and malignant tumor cell fraction. Specimen adequacy findings should be documented in the patient report.

It is critical that pathologists selecting blocks for biomarker testing understand the specimen requirements of the method being employed in terms of total tissue amount (a reflection of the total amount of DNA required for the assays) and the fraction of malignant tumor cells in the specimen focus to be evaluated. The total amount of tissue selected for evaluation is significant in two respects. First, the amount of tissue sampled should be of sufficient quantity to produce a result that is reliably representative of the entire tumor. While recent evidence indicates that some genes continue to evolve during tumor progression, leading to substantial tumor genetic heterogeneity, those driver mutations of importance to CRC are usually, but not always, homogeneous throughout the tumor. The amount of tumor necessary, however, for a particular analytical method can vary and demands knowledge and due attention to the indicated tissue requirements for the specific assay employed. The minimal required proportion of tumor DNA in a sample from cancer is dictated by the analytical sensitivity of the particular validated assay. As shown in Table 13, the amount of tumor used in the analyses of KRAS mutations in several studies comparing the test-performing characteristics of various assays varied widely, ranging from $1 \%$ to $90 \%$.

The proportion of malignant tumor cells (as opposed to tumor-associated nonmalignant cells, eg, stromal fibroblasts, endothelial cells, infiltrating inflammatory cells) should be evaluated as accurately as possible and documented. This evaluation is most readily performed by estimating the proportion of malignant cell nuclei to nonmalignant cell nuclei within the focus selected for evaluation. ${ }^{143}$ Understanding that the number of mutated alleles for a particular gene may represent as few as half of the alleles in diploid tumor cells, a tumor cell focus with a nominal proportion of $50 \%$ tumor cells would have a mutant allele fraction of $25 \%$, a value approaching the analytical sensitivity of some molecular assays. So, while a variety of molecular methods can be used to evaluate tissue specimens, it is critical that these be carefully matched to their specific tissue and tumor cell proportion requirements. When adhered to, all of these methods can produce accurate and reliable results.

Pathologists evaluating tissue section for biomarker evaluation should also be aware that necrosis and tissue degeneration can lead to erroneous results, and foci demonstrating significant necrosis should be avoided for molecular testing. Any amount of necrosis in the sample selected for biomarker testing should be estimated and documented.

18. Expert Consensus Opinion.-Laboratories should use colorectal carcinoma molecular biomarker testing methods that are able to detect mutations in specimens with at least $5 \%$ mutant allele frequency, taking into account the analytical sensitivity of the assay (limit of detection or LOD) and tumor enrichment (eg, microdissection).

Note: It is recommended that the operational minimal neoplastic carcinoma cell content tested should be set at least two times the assay's LOD.

Since the accuracy and results of testing for molecular markers are dependent on both tumor cell content and the assay-specific sensitivity in the identification of a mutant allele against a background of wild-type/nonmutated alleles, it is suggested that laboratories should establish minimum acceptable tumor cell content as a component of their specimen requirements. It is recommended that a pathologist reviews all cases for tumor cell content and quality. Due to the stochastic nature of mutant allele identification at the lower LOD, it is recommended that the minimal tumor cell content be at least two times the lower LOD of a validated molecular method or assay. This LOD was also recommended for RAS testing of colorectal carcinoma in the guidance document from the United Kingdom. ${ }^{126}$ Hence, if a particular assay has a lower limit of mutant allele detection of $5 \%$, then the minimum tumor cell content in samples analyzed by this assay should be at least $10 \%$ to reliably detect heterozygous mutations in those neoplasms. Due to intratumoral heterogeneity, subclones, and the nature of tissue sampling, clinical trials have used $5 \%$ as the lower 
LOD, and for clinical purposes, it is recommended that the lower LOD for a mutant allele be at least $5 \% .{ }^{12}$ Therefore, the utilization of methods such as PCR, HRM, single-strand conformation polymorphism, pyrosequencing, or commercially available kits that achieve this level of sensitivity is recommended ${ }^{130,137,138,142}$ (Table 13).

This recommendation is supported by four prospective cohort studies $130,137,138,142$ and two retrospective cohort studies. ${ }^{102,144}$ The four prospective cohort studies all studied a single cohort, allowing for within-group comparisons. For this reason, all were balanced between comparison groups, and no adjustments were needed to account for baseline differences. All four reported nonindustry funding, and all were deemed to have a low risk of bias.

The two retrospective cohort studies ${ }^{102,144}$ also used single cohorts, allowing for within-group comparisons only. One ${ }^{102}$ did not report the source of funding, while the other ${ }^{144}$ reported nonindustry funding. Both were deemed to have a low risk of bias.

None of the studies had methodologic flaws that would raise concerns about their findings.

19. Expert Consensus Opinion.-Colorectal carcinoma molecular biomarker results should be made available as promptly as feasible to inform therapeutic decision making, both prognostic and predictive.

Note: It is suggested that a benchmark of $90 \%$ of reports be available within 10 working days from date of receipt in the molecular diagnostics laboratory.

Combined chemotherapy, including anti-EGFR therapy, in patients with CRC in the absence of mutations in the EGFR signaling pathway is associated with significant survival advantage. No significant therapeutic benefit is derived from anti-EGFR therapy in the presence of mutations in KRAS and NRAS. ${ }^{44}$ The presence of deficient MMR in stage II CRC indicates a good prognosis and identifies patients for whom adjuvant 5 -fluorouracil monobased therapies have no significant benefit. ${ }^{145,146}$ The presence of deficient MMR or BRAF p.V600E mutation in proficient MMR CRCs has important prognostic significance. ${ }^{54}$

In the absence of published data establishing an evidencebased recommendation, it is our expert consensus opinion that the above results, regardless of testing methods, be available from test ordering in the initial diagnostic pathology laboratory to the clinical team within 2 weeks (10 working days). The 10 working days does not include the time before the tissue specimen is available for testing (ie, from diagnostic procedure to receipt in laboratory) or time to retrieve tissue samples from an outside laboratory. Laboratories unable to maintain this standard, either through in-house testing or use of a reference laboratory, need to implement measures to improve test result turnaround time. A turnaround time of 7 working days was recommended for RAS testing of colorectal carcinoma in the guidance document from the Association of Clinical Pathologists Molecular Pathology and Diagnostics Group in the United Kingdom. ${ }^{126}$

This recommendation is supported by evidence from one randomized controlled trial, reported by Douillard et al. ${ }^{44}$ This report used prospective patient data collected within the PRIME trial. While it did not report details on the randomization, blinding, statistical power calculation, sample size, or length of follow-up, it did report on baseline characteristics and was otherwise well reported. Funding was reported to be partially from industry sources. Overall, this trial was found to have a low to moderate risk of bias and did not have methodologic flaws that would raise concerns about its findings.

Each laboratory should develop a quality assurance program to monitor turnaround times for all cancer therapeutic and prognostic biomarkers.

20. Expert Consensus Opinion.-Colorectal carcinoma molecular biomarker testing reports should include a results and interpretation section readily understandable by oncologists and pathologists. Appropriate Human Genome Variation Society (HGVS) and HUGO nomenclature must be used in conjunction with any historical genetic designations.

Reporting of molecular results is becoming more complex as new information and clinical utility are discovered for somatic variants. Single-gene assays are still being widely used, but multiplexing has allowed for multiple possible results. With the introduction of NGS into the clinical setting, multiple somatic mutations with clinical significance may be identified. However, panel assays by NGS can also reveal variants with unknown clinical significance. As pathogenic genes and somatic mutations have been discovered during the past 30 years, there has been divergent nomenclature employed, making clinical reporting and clinical analysis difficult. Presently and in the future, as national databases are constructed annotating clinical somatic variants, it is imperative that standardized nomenclature be employed to identify the clinical significance of rare variants.

Clinicians want a report that is easily readable and understandable but that gives pertinent clinical information concisely, accurately, and thoroughly. Reported variants should be identified using both DNA and protein nomenclature. Citing codon positivity only is not encouraged (eg, positive for a KRAS codon 12 mutation). The specific mutation should be explained using standardized nomenclature, preferably HUGO gene nomenclature. ${ }^{112,147}$ Historical designations (eg, historical HER-2/neu, for HUGO $E R B B 2$ ) should also be included as appropriate in the report to avoid confusion among oncologists. Importantly, the messenger RNA transcript number (NM_\#) from the NCBI, used to designate the specific codon numbering, should be named in the report since numbering can differ between the different/alternative transcript designations for the same gene. If using NGS, variants should at least be classified as pathogenic, likely pathogenic, variant of unknown significance, likely benign, or benign, but classification of somatic mutations is still awaiting specifically approved guidelines. ${ }^{148}$ However, a numerical classification scheme for somatic variants has been proposed, taking into consideration actionability of the variant in the patient's tumor type versus other tumor types, predicted pathogenicity (using programs such as SIFT and PolyPhen 2) in the patient's tumor type versus other tumor types, variant recurrence in a certain cancer type, or unknown significance. ${ }^{149}$ Such a classification scheme may be better suited to somatic variants considering the indications for which most of these assays are being ordered.

Reports should contain the analytical result, the method used, and information about the genes and loci tested or included in the assay; the assay limit of detection; and any required disclaimers (eg, ASR) that are required to meet regulations. When reasonable and applicable, an interpretive comment should be given to ensure that results are correctly understood. ${ }^{112}$ Such an interpretive comment may 
include information regarding therapeutic implications, prognostic implications, and/or pathogenic significance of the mutation and, when appropriate or desired, potential applicable clinical trials.

In summary, molecular reports should be easily understandable by clinical oncologists and use standardized nomenclature outlined by HGVS/HUGO. All reports should contain the elements of result, interpretation, variant classification, and information as applicable; limit of detection of the assay and methods to assist the oncologist in understanding the test result; and limitations as they consider the result in a clinical context.

21. Strong Recommendation.-Laboratories must incorporate colorectal carcinoma molecular biomarker testing methods into their overall laboratory quality improvement program, establishing appropriate quality improvement monitors as needed to ensure consistent performance in all steps of the testing and reporting process. In particular, laboratories performing colorectal carcinoma molecular biomarker testing must participate in formal proficiency testing programs, if available, or an alternative proficiency assurance activity.

Proficiency testing (PT) is an important component of quality assurance for laboratory tests in general and applies to the molecular tests discussed in the current CRC molecular testing guidelines. These include mutational as well as immunohistochemical testing. Participation in PT allows the assessment and comparison of test performance among different clinical laboratories and technologies and allows verification of accuracy and reliability of laboratory tests. ${ }^{150}$

From a regulatory standpoint, PT in the United States is a requirement for accreditation by the Centers for Medicare \& Medicaid Services. Participation in PT may be done through CAP PT programs or through other providers accepted by CLIA. ${ }^{151}$ Other countries-namely, the United Kingdomfollow similar guidelines, recommending that laboratories providing RAS testing of CRC should demonstrate successful participation in a relevant external quality assurance scheme and be appropriately accredited. ${ }^{126}$

Formal external proficiency testing programs for analytes other than KRAS, MSI, MMR, and BRAF may not be available at the time of this publication. Alternative proficiency testing activities should be used. Appropriate alternative performance assessment procedures may include split sample analysis with other laboratories or, if that is not available, assessment of split samples with an established in-house method and previously assayed material, which are run and interpreted by laboratory personnel who do not have access to the prior results. ${ }^{151}$ If exchanging specimens with other laboratories is the laboratory proficiency approach, this should be done with one or more other laboratories at least twice per year. ${ }^{105}$ Methods-based proficiency testing (MBPT) refers to a testing approach that is based on method, rather than based on each individual analyte tested. MBPT is well established for several pathology subspecialty areas, and the concept of MBPT complies with federal laboratory regulations. ${ }^{151}$

\section{DISCUSSION ON EMERGING BIOMARKERS}

Numerous studies have reported potential molecular biomarkers for CRC prognosis, while fewer studies evaluated markers that could be predictive of response to specific treatments. Many published studies are limited due to early exploratory and retrospective analyses, and those biomarkers, while of potential interest, have not made it to clinical practice. Our systematic review identified several CRC molecular biomarkers that showed either prognostic or treatment predictive characteristics in single studies (Supplemental Table 15). Most of the molecular biomarkers reported in the studies listed in the Supplemental Table 15 were tested for expression by immunohistochemistry. Immunohistochemistry is notable for its widespread availability in pathology laboratories but has limited quantitative capabilities due to difficult standardization of quantitative or semiquantitative scoring, and is fraught by significant interobserver variability. A problem of quantitative assays, such as gene expression, microRNA expression, and methylation levels, tested in solid tumors, results from the intrinsic mixed nature of the tissue with significant variability of tumor and nontumor tissue content. Another limitation of molecular biomarker discovery approaches that rely on expression levels is that these biomarkers have not been evaluated in the context of complex molecular regulation of individual cancer subtypes. Their fruitful use in the clinic may require further studies that take into account computational predictions of biological behavior and validation in prospective cohorts.

A great deal of interest has been raised recently for noninvasive prognostic and/or therapy-predictive molecular biomarkers, such as those tested in circulating tumor cells or circulating nucleic acids, either as free nucleic acid in serum or associated with extracellular vesicles or exosomes. This has been referred to as "liquid biopsy."152 Liquid biopsies may be particularly useful in the management of patients with CRC to identify recurrence, RAS mutation testing for emergence of treatment resistance associated with antiEGFR therapy, and potential early cancer detection in defined subpopulations, such as those at high risk of CRC. Overall, molecular biomarkers for colorectal cancer tested in liquid biopsy samples are promising but await further validation.

Emerging data indicate that MMR status may have predictive value in some settings, specifically in patients with advanced disease being considered for anti-PD-1/PDL1 therapy. ${ }^{68,69}$

\section{CONCLUSIONS}

Evidence supports mutational testing of specific genes in the EGFR signaling pathway, since they provide clinically actionable information for targeted therapy of CRC with anti-EGFR monoclonal antibodies. Mutations in some of the biomarkers have clear prognostic value (BRAF, MMR), and at least two (KRAS and NRAS) have relatively strong evidence as negative predictors of benefit to anti-EGFR therapies and should be used to guide the use of these agents. BRAF mutations are consistently associated with poor outcomes in patients with metastatic CRC, including those who relapse after adjuvant therapy. Patients with localized colon cancer and dMMR have improved outcomes. Emerging data suggest that MMR status has predictive value in some settings, specifically in patients with advanced disease being considered for anti-PD-1/PD-L1 therapy.

Laboratory approaches to operationalize molecular testing for predictive and prognostic molecular biomarkers involve selection of assays, type of specimens to be tested, timing of ordering of tests, and turnaround time for testing results. A number of alternative technical approaches can effectively 
be used as long as test specificity and sensitivity meet the clinical needs. While earlier testing approaches were focused on one or a few testing targets (eg, BRAF p.V600 mutations), new approaches are currently using gene panels such as targeted NGS cancer panels, which can range from a few to hundreds of genes and amplicons with known mutational hotspots in cancer.

These guidelines will be subjected to regular updates, such that new advances in the field can be captured and integrated in the guidelines in a timely manner.

The authors gratefully acknowledge the following: expert and advisory panel members Kevin Halling, MD, Charles Blanke, MD, Pr. Jean-François Fléjou, Heather Hampel, MS, CGC, J. Randolph Hecht, MD, Kazunori Kanehira, MD, Faye Kastrinos, MD, MPH, Carla B. MacLeod, MD, Peter J. Odwyer, MD, Weijing Sun, MD, Josep Tabernero, MD, PhD, Laura H. Tang, MD, PhD, Mary Kay Washington, MD, PhD, Shuji Ogino, MD, PhD, MS, Loren Joseph, MD, Kim Ryan, and Pamela McAllister, PhD, for their review of the key questions, recommendations, and draft manuscript, as well as Kaitlin Einhaus, Lisa A. Fatheree, SCT(ASCP), Jeff Jacobs, MA, Sandra Larsen, MBA, MT(ASCP), Melvin Limson, PhD, John Olsen, MD, Mrudula Pullambhatla, MS, Shiwen Song, MD, Megan Wick, MT(ASCP), and Mary Steele Williams, MNA, MT(ASCP)SM, $\mathrm{CAE}$, for their support throughout the guideline development process.

\section{References}

1. Febbo PG, Ladanyi M, Aldape KD, et al. NCCN task force report: evaluating the clinical utility of tumor markers in oncology. J Natl Compr Cancer Netw. 2011;9(suppl 5):S1-S33.

2. Grothey A. EGFR antibodies in colorectal cancer: where do they belong? Clin Oncol. 2010;28:4668-4670. doi 10.1200/JCO.2010.29.3407

3. De Roock W, Claes B, Bernasconi D, et al. Effects of KRAS, BRAF, NRAS and PIK3CA mutations on the efficacy of cetuximab plus chemotherapy in chemotherapy-refractory metastatic colorectal cancer: a retrospective consortium analysis. Lancet Oncol. 2010;11:753-762. doi 10.1016/S1470-2045(10)70130-3

4. De Roock W, De Vriendt V, Normanno N, et al. KRAS, BRAF, PIK3CA, and PTEN mutations: implications for targeted therapies in metastatic colorectal cancer. Lancet Oncol. 2011;12:594-603. doi 10.1016/S1470-2045(10)70209-6

5. Rubenstein JH, Enns R, Heidelbaugh J, et al. American Gastroenterological Association Institute guideline on the diagnosis and management of Lynch syndrome. Gastroenterology. 2015;149:777-782. doi10.1053/j.gastro.2015.07.036

6. Des Guetz G, Schischmanoff O, Nicolas P, et al. Does microsatellite instability predict the efficacy of adjuvant chemotherapy in colorectal cancer? A systematic review with meta-analysis. Eur J Cancer. 2009;45:1890-1896. doi 10. 1016/j.ejca.2009.04.018

7. Guastadisegni C, Colafranceschi M, Ottini L, et al. Microsatellite instability as a marker of prognosis and response to therapy: a meta-analysis of colorecta cancer survival data. Eur J Cancer. 2010;46:2788-2798. doi 10.1016/j.ejca.2010. 05.009

8. Gavin PG, Colangelo LH, Fumagalli D, et al. Mutation profiling and microsatellite instability in stage II and III colon cancer: an assessment of their prognostic and oxaliplatin predictive value. Clin Cancer Res. 2012;18:65316541. doi 10.1158/1078-0432.CCR-12-0605

9. Institute of Medicine. Clinical Practice Guidelines We Can Trust. Washington, DC: National Academies Press; 2011.

10. National Health and Medical Research Council. A guide to the development, implementation and evaluation of clinicl practice guidelines. 1999. https://www.nhmrc.gov.au/_files_nhmrc/publications/attachments/cp.30. pdf. Accessed March 23, 2016.

11. Guyatt GH, Oxman AD, Vist GE, et al; GRADE Working Group. GRADE: an emerging consensus on rating quality of evidence and strength of recommendations. BMJ. 2008;336:924-926. doi 10.1136/bmj.39489.470347. $\mathrm{AD}$

12. Sorich MJ, Wiese MD, Rowland A, et al. Extended RAS mutations and anti-EGFR monoclonal antibody survival benefit in metastatic colorectal cancer: a meta-analysis of randomized, controlled trials. Ann Oncol. 2015;26:13-21. doi 10.1093/annonc/mdu378

13. Ibrahim EM, Abouelkhair KM. Clinical outcome of panitumumab for metastatic colorectal cancer with wild-type KRAS status: a meta-analysis of randomized clinical trials. Med Oncol. 2011;28(suppl 1):S310-S317. doi 10. 1007/s12032-010-9760-4

14. Modest DP, Brodowicz T, Stintzing $S$, et al. Impact of the specific mutation in KRAS codon 12 mutated tumors on treatment efficacy in patients with metastatic colorectal cancer receiving cetuximab-based first-line therapy: a pooled analysis of three trials. Oncology. 2012;83:241-247. doi 10.1159/ 000339534

Arch Pathol Lab Med-Vol 141, May 2017
15. Petrelli F, Borgonovo K, Cabiddu M, et al. Cetuximab and panitumumab in KRAS wild-type colorectal cancer: a meta-analysis. Int J Colorectal Dis. 2011; 26:823-833. doi 10.1007/s00384-011-1149-0

16. Zhou SW, Huang YY, Wei Y, et al. No survival benefit from adding cetuximab or panitumumab to oxaliplatin-based chemotherapy in the first-line treatment of metastatic colorectal cancer in KRAS wild type patients: a metaanalysis. PLoS One. 2012;7:e50925. doi 10.1371/journal.pone.0050925

17. Karapetis CS, Khambata-Ford S, Jonker DJ, et al. K-ras mutations and benefit from cetuximab in advanced colorectal cancer. N Engl J Med. 2008;359: 1757-1765. doi 10.1056/NEJMoa0804385

18. Adelstein BA, Dobbins TA, Harris CA, et al. A systematic review and meta-analysis of KRAS status as the determinant of response to anti-EGFR antibodies and the impact of partner chemotherapy in metastatic colorectal cancer. Eur J Cancer. 2011;47:1343-1354. doi 10.1016/j.ejca.2011.03.031

19. Allegra CJ, Jessup JM, Somerfield MR, et al. American Society of Clinical Oncology provisional clinical opinion: testing for KRAS gene mutations in patients with metastatic colorectal carcinoma to predict response to antiepidermal growth factor receptor monoclonal antibody therapy. J Clin Oncol. 2009;27:2091-2096. doi 10.1200/JCO.2009.21.9170

20. Baas JM, Krens LL, Guchelaar HJ, et al. Concordance of predictive markers for EGFR inhibitors in primary tumors and metastases in colorectal cancer: a review. Oncologist. 2011;16:1239-1249. doi 10.1634/theoncologist. 2011-0024

21. Chen J, Ye Y, Sun H, et al. Association between KRAS codon 13 mutations and clinical response to anti-EGFR treatment in patients with metastatic colorectal cancer: results from a meta-analysis. Cancer Chemother Pharmacol. 2013;71:265-272. doi 10.1007/s00280-012-2005-9

22. Dahabreh IJ, Terasawa T, Castaldi PJ, et al. Systematic review: antiepidermal growth factor receptor treatment effect modification by KRAS mutations in advanced colorectal cancer. Ann Intern Med. 2011;154:37-49. doi 10.7326/0003-4819-154-1-201101040-00006

23. De Roock W, Jonker DJ, Di Nicolantonio F, et al. Association of KRAS p.G13D mutation with outcome in patients with chemotherapy-refractory metastatic colorectal cancer treated with cetuximab. JAMA. 2010;304:18121820. doi 10.1001/jama.2010.1535

24. Health Quality Ontario. KRAS testing for Anti-EGFR therapy in advanced colorectal cancer: an evidence-based and economic analysis. Ont Health Technol Assess Ser. 2010;10:1-49.

25. Hoyle M, Crathorne L, Peters J, et al. The clinical effectiveness and costeffectiveness of cetuximab (mono- or combination chemotherapy), bevacizumab (combination with non-oxaliplatin chemotherapy) and panitumumab (monotherapy) for the treatment of metastatic colorectal cancer after first-line chemotherapy (review of technology appraisal No.150 and part review of technology appraisal No. 118): a systematic review and economic model. Health Technol Assess. 2013; 17:1-237.

26. Ibrahim EM, Zekri JM, Bin Sadiq BM. Cetuximab-based therapy for metastatic colorectal cancer: a meta-analysis of the effect of K-ras mutations. Int Colorectal Dis. 2010;25:713-721. doi 10.1007/s00384-010-0927-4

27. Jiang Z, Li C, Li F, et al. EGFR gene copy number as a prognostic marker in colorectal cancer patients treated with cetuximab or panitumumab: a systematic review and meta analysis. PLoS One. 2013;8:e56205. doi 10.1371/journal.pone. 0056205

28. Ku GY, Haaland BA, de Lima Lopes G Jr. Cetuximab in the first-line treatment of K-ras wild-type metastatic colorectal cancer: the choice and schedule of fluoropyrimidine matters. Cancer Chemother Pharmacol. 2012;70: 231-238. doi 10.1007/s00280-012-1898-7

29. Lin AY, Buckley NS, Lu AT, et al. Effect of KRAS mutational status in advanced colorectal cancer on the outcomes of anti-epidermal growth factor receptor monoclonal antibody therapy: a systematic review and meta-analysis. Clin Colorectal Cancer. 2011;10:63-69. doi 10.3816/CCC.2011.n.009

30. Linardou H, Dahabreh IJ, Kanaloupiti D, et al. Assessment of somatic kRAS mutations as a mechanism associated with resistance to EGFR-targeted agents: a systematic review and meta-analysis of studies in advanced non-smallcell lung cancer and metastatic colorectal cancer. Lancet Oncol. 2008;9:962972. doi 10.1016/S1470-2045(08)70206-7

31. Loupakis F, Cremolini C, Salvatore L, et al. Clinical impact of antiepidermal growth factor receptor monoclonal antibodies in first-line treatment of metastatic colorectal cancer: meta-analytical estimation and implications for therapeutic strategies. Cancer. 2012;118:1523-1532. doi 10.1002/cncr. 26460

32. Mao C, Huang YF, Yang ZY, et al. KRAS p.G13D mutation and codon 12 mutations are not created equal in predicting clinical outcomes of cetuximab in metastatic colorectal cancer: a systematic review and meta-analysis. Cancer. 2013;119:714-721. doi 10.1002/cncr.27804

33. Mao C, Yang ZY, Hu XF, et al. PIK3CA exon 20 mutations as a potential biomarker for resistance to anti-EGFR monoclonal antibodies in KRAS wild-type metastatic colorectal cancer: a systematic review and meta-analysis. Ann Oncol. 2012;23:1518-1525. doi 10.1093/annonc/mdr464

34. Petrelli F, Barni S. Resectability and outcome with anti-EGFR agents in patients with KRAS wild-type colorectal liver-limited metastases: a meta-analysis. Int J Colorectal Dis. 2012;27:997-1004. doi 10.1007/s00384-012-1438-2

35. Petrelli F, Coinu A, Cabiddu M, et al. KRAS as prognostic biomarker in metastatic colorectal cancer patients treated with bevacizumab: a pooled analysis of 12 published trials. Med Oncol. 2013;30:650. doi 10.1007/s12032 013-0650-4 
36. Qiu LX, Mao C, Zhang J, et al. Predictive and prognostic value of KRAS mutations in metastatic colorectal cancer patients treated with cetuximab: a meta-analysis of 22 studies. Eur J Cancer. 2010;46:2781-2787. doi 10.1016/j. ejca.2010.05.022

37. Ren J, Li G, Ge J, et al. Is K-ras gene mutation a prognostic factor for colorectal cancer: a systematic review and meta-analysis. Dis Colon Rectum. 2012;55:913-923. doi 10.1097/DCR.0b013e318251d8d9

38. Tsoukalas N, Tzovaras AA, Tolia M, et al. Meta-analysis of the predictive value of KRAS mutations in treatment response using cetuximab in colorectal cancer. J BUON. 2012;17:73-78.

39. Vale CL, Tierney JF, Fisher D, et al. Does anti-EGFR therapy improve outcome in advanced colorectal cancer? A systematic review and meta-analysis. Cancer Treat Rev. 2012;38:618-625. doi 10.1016/j.ctrv.2011.11.002

40. Yang ZY, Shen WX, Hu XF, et al. EGFR gene copy number as a predictive biomarker for the treatment of metastatic colorectal cancer with anti-EGFR monoclonal antibodies: a meta-analysis. J Hematol Oncol. 2012;5:52. doi 10. 1186/1756-8722-5-52

41. Zhang L, Ma L, Zhou Q. Overall and KRAS-specific results of combined cetuximab treatment and chemotherapy for metastatic colorectal cancer: a metaanalysis. Int J Colorectal Dis. 2011;26:1025-1033. doi 10.1007/s00384-0111197-5

42. Ross JS. Clinical implementation of KRAS testing in metastatic colorectal carcinoma: the pathologist's perspective. Arch Pathol Lab Med. 2012;136:12981307. doi 10.5858/arpa.2011-0478-RA

43. Bando $H$, Yoshino $T$, Shinozaki $E$, et al. Simultaneous identification of 36 mutations in KRAS codons 61 and 146, BRAF, NRAS, and PIK3CA in a single reaction by multiplex assay kit. BMC Cancer. 2013;13:405. doi 10.1186/14712407-13-405

44. Douillard JY, Oliner KS, Siena S, et al. Panitumumab-FOLFOX4 treatment and RAS mutations in colorectal cancer. N Engl J Med. 2013;369:1023-1034. doi 10.1056/NEJMoa1305275

45. Etienne-Grimaldi MC, Mahamat A, Chazal M, et al. Molecular patterns in deficient mismatch repair colorectal tumours: results from a French prospective multicentric biological and genetic study. Br J Cancer. 2014;110:2728-2737. doi 10.1038/bjc.2014.213

46. Van Cutsem E, Lenz $\mathrm{HJ}$, Kohne $\mathrm{CH}$, et al. Fluorouracil, leucovorin, and irinotecan plus cetuximab treatment and RAS mutations in colorectal cancer. Clin Oncol. 2015;33:692-700. doi 10.1200/JCO.2014.59.4812

47. Xu Q, Xu AT, Zhu MM, et al. Predictive and prognostic roles of BRAF mutation in patients with metastatic colorectal cancer treated with anti-epiderma growth factor receptor monoclonal antibodies: a meta-analysis. J Dig Dis. 2013; 14:409-416. doi 10.1111/1751-2980.12063]

48. Yuan ZX, Wang XY, Qin QY, et al. The prognostic role of BRAF mutation in metastatic colorectal cancer receiving anti-EGFR monoclonal antibodies: a meta-analysis. PLoS One. 2013;8:e65995. doi 10.1371/journal.pone.0065995

49. Forbes SA, Bhamra G, Bamford S, et al. The catalogue of somatic mutations in cancer (COSMIC). Curr Protoc Hum Genet. 2008; Chapter 10:Unit 10.11.

50. Lin JS, Webber EM, Senger CA, et al. Systematic review of pharmacogenetic testing for predicting clinical benefit to anti-EGFR therapy in metastatic colorectal cancer. Am J Cancer Res. 2011;1:650-662.

51. Mao C, Liao RY, Qiu LX, et al. BRAF V600E mutation and resistance to antiEGFR monoclonal antibodies in patients with metastatic colorectal cancer: a metaanalysis. Mol Biol Rep. 2011;38:2219-2223. doi 10.1007/s11033-010-0351-4

52. Parsons MT, Buchanan DD, Thompson B, et al. Correlation of tumour BRAF mutations and MLH1 methylation with germline mismatch repair (MMR) gene mutation status: a literature review assessing utility of tumour features for MMR variant classification. J Med Genet. 2012;49:151-157. doi 10.1136/ jmedgenet-2011-100714]

53. Cui D, Cao D, Yang Y, et al. Effect of BRAF V600E mutation on tumor response of anti-EGFR monoclonal antibodies for first-line metastatic colorectal cancer treatment: a meta-analysis of randomized studies. Mol Biol Rep. 2014;41: 1291-1298 doi 10.1007/s11033-013-2974-8

54. Lochhead P, Kuchiba A, Imamura $Y$, et al. Microsatellite instability and BRAF mutation testing in colorectal cancer prognostication. J Natl Cancer Inst. 2013;105:1151-1156. doi 10.1093/jnci/djt173

55. Rowland A, Dias MM, Wiese MD, et al. Meta-analysis of BRAF mutation as a predictive biomarker of benefit from anti-EGFR monoclonal antibody therapy for RAS wild-type metastatic colorectal cancer. Br J Cancer. 2015;112:18881894. doi 10.1038/bjc.2015.173

56. Cremolini C, Loupakis F, Antoniotti C, et al. FOLFOXIRI plus bevacizumab versus FOLFIRI plus bevacizumab as first-line treatment of patients with metastatic colorectal cancer: updated overall survival and molecular subgroup analyses of the open-label, phase 3 TRIBE study. Lancet Oncol. 2015;16:13061315. doi 10.1016/S1470-2045(15)00122-9

57. Corcoran RB, Atreya CE, Falchook GS, et al. Phase 1-2 trial of the BRAF inhibitor dabrafenib (D) plus MEK inhibitor trametinib (T) in BRAF V600 mutant colorectal cancer (CRC): updated efficacy and biomarker analysis [ASCO meeting abstract 3517]. J Clin Oncol. 2014;32:5s. http://meetinglibrary.asco. org/content/131743-144. Accessed November 18, 2016.

58. Bendell JC, Atreya CE, André T. Efficacy and tolerability in an open-label phase I/II study of MEK inhibitor trametinib (T) BRAF inhibitor dabrafenib (D), and anti-EGFR antibody panitumumab $(\mathrm{P})$ in combination in patients (pts) with BRAF V600E mutated colorectal cancer (CRC) [ASCO meeting abstract 3515]. J Clin Oncol. 2014;32:5s. http://meetinglibrary.asco.org/content/131642-144. Accessed November 18, 2016.
59. Temraz S, Mukherii D, Shamseddine A. Dual inhibition of MEK and PI3K pathway in KRAS and BRAF mutated colorectal cancers. Int J Mol Sci. 2015;16: 22976-22988. doi 10.3390/ijms160922976

60. Taieb J, Zaanan A, Le Malicot K, et al. Prognostic effect of BRAF and KRAS mutations in patients with stage III colon cancer treated with leucovorin, fluorouracil, and oxaliplatin with or without cetuximab: a post hoc analysis of the PETACC-8 trial [published online January 14, 2016]. JAMA Oncol. doi 10.1001/ jamaoncol.2015.5225

61. Barras D. BRAF mutation in colorectal cancer: an update. Biomark Cancer. 2015;7:9-12. doi 10.4137/BIC.S25248

62. Geiersbach KB, Samowitz WS. Microsatellite instability and colorectal cancer. Arch Pathol Lab Med. 2011;135:1269-1277. doi 10.5858/arpa.20110035-RA

63. Barrow E, Hill J, Evans DG. Cancer risk in lynch syndrome. Fam Cancer. 2013;12:229-240. doi 10.1007/s10689-013-9615-1

64. Schmeler KM, Lynch HT, Chen LM, et al. Prophylactic surgery to reduce the risk of gynecologic cancers in the Lynch syndrome. N Engl J Med. 2006;354: 261-269. doi 10.1056/NEJMoa052627

65. Vasen HF, Blanco I, Aktan-Collan K, et al. Revised guidelines for the clinical management of Lynch syndrome (HNPCC): recommendations by a group of European experts. Gut. 2013;62:812-823. doi 10.1136/gutjnl-2012-304356

66. Funkhouser WK Jr, Lubin IM, Monzon FA, et al. Relevance, pathogenesis, and testing algorithm for mismatch repair-defective colorectal carcinomas: a report of the Association for Molecular Pathology. J Mol Diagn. 2012;14:91-103. doi 10.1016/j.jmoldx.2011.11.001

67. Kawakami H, Zaanan A, Sinicrope FA. Implications of mismatch repairdeficient status on management of early stage colorectal cancer. J Gastrointest Oncol. 2015;6:676-684.

68. Diaz LA Jr, Le DT. PD-1 blockade in tumors with mismatch-repair deficiency. N Engl J Med. 2015;373:1979. doi 10.1056/NEJMc1510353

69. Le DT, Uram JN, Wang H, et al. PD-1 blockade in tumors with mismatchrepair deficiency. N Engl J Med. 2015;372:2509-2520. [PMC][10.1056/ NEJMoa1500596

70. Le DT, Uram JN, Wang $\mathrm{H}$, et al. Programmed death-1 blockade in mismatch repair deficient colorectal cancer [ASCO meeting abstract 103]. J Clin Oncol. 2016;34. http://meetinglibrary.asco.org/content/167415-176. Accessed November 18, 2016.

71. Yang $Z Y, W u X Y$, Huang $Y F$, et al. Promising biomarkers for predicting the outcomes of patients with KRAS wild-type metastatic colorectal cancer treated with anti-epidermal growth factor receptor monoclonal antibodies: a systematic review with meta-analysis. Int J Cancer. 2013;133:1914-1925 doi 10.1002/ijc. 28153

72. Pietrantonio F, Petrelli F, Coinu A, et al. Predictive role of BRAF mutations in patients with advanced colorectal cancer receiving cetuximab and panitumumab: a meta-analysis. Eur J Cancer. 2015;51:587-594. doi 10.1016/j.ejca.2015. 01.054

73. Wu S, Gan Y, Wang X, et al. PIK3CA mutation is associated with poor survival among patients with metastatic colorectal cancer following anti-EGFR monoclonal antibody therapy: a meta-analysis. J Cancer Res Clin Oncol. 2013; 139:891-900. doi 10.1007/s00432-013-1400-x

74. Karapetis CS, Jonker D, Daneshmand $M$, et al. PIK3CA, BRAF, and PTEN status and benefit from cetuximab in the treatment of advanced colorectal cancer-results from NCIC CTG/AGITG CO.17. Clin Cancer Res. 2014;20:744753. doi 10.1158/1078-0432.CCR-13-0606

75. Liao X, Morikawa T, Lochhead P, et al. Prognostic role of PIK3CA mutation in colorectal cancer: cohort study and literature review. Clin Cancer Res. 2012;18:2257-2268. [doi 10.1158/1078-0432.CCR-11-2410

76. Ogino S, Liao X, Imamura $Y$, et al. Predictive and prognostic analysis of PIK3CA mutation in stage III colon cancer intergroup trial. J Natl Cancer Inst. 2013;105:1789-1798. doi 10.1093/jnci/djt298

77. Ogino S, Nosho K, Kirkner GJ, et al. PIK3CA mutation is associated with poor prognosis among patients with curatively resected colon cancer. J Clin Oncol. 2009;27:1477-1484. doi 10.1200/JCO.2008.18.6544

78. Flossmann E, Rothwell PM. Effect of aspirin on long-term risk of colorectal cancer: consistent evidence from randomised and observational studies. Lancet. 2007;369:1603-1613. doi 10.1016/S0140-6736(07)60747-8

79. Jacobs EJ, Thun MJ, Bain EB, et al. A large cohort study of long-term daily use of adult-strength aspirin and cancer incidence. J Natl Cancer Inst. 2007;99: 608-615. doi 10.1093/jnci/djk132

80. Chan AT, Giovannucci EL, Meyerhardt JA, et al. Aspirin dose and duration of use and risk of colorectal cancer in men. Gastroenterology. 2008;134:21-28. doi 10.1053/j.gastro.2007.09.035

81. Domingo E, Church DN, Sieber O, et al. Evaluation of PIK3CA mutation as a predictor of benefit from nonsteroidal anti-inflammatory drug therapy in colorectal cancer. J Clin Oncol. 2013;31:4297-4305. doi 10.1200/JCO.2013.50. 0322

82. Liao X, Lochhead P, Nishihara R, et al. Aspirin use, tumor PIK3CA mutation, and colorectal-cancer survival. N Engl J Med. 2012;367:1596-1606. doi 10.1056/NEJMoa1207756

83. Kothari N, Kim R, Jorissen RN, et al. Impact of regular aspirin use on overall and cancer-specific survival in patients with colorectal cancer harboring a PIK3CA mutation. Acta Oncol. 2015;54:487-492. doi 10.3109/0284186X.2014. 990158

84. Berg M, Danielsen SA, Ahlquist $T$, et al. DNA sequence profiles of the colorectal cancer critical gene set KRAS-BRAF-PIK3CA-PTEN-TP53 related to 
age at disease onset. PLoS One. 2010;5:e13978. doi 10.1371/journal.pone. 0013978

85. Shen $Y$, Yang J, Xu Z, et al. Phosphatase and tensin homolog expression related to cetuximab effects in colorectal cancer patients: a meta-analysis. World J Gastroenterol. 2012;18:2712-2718. doi 10.3748/wjg.v18.i21.2712

86. Wang ZH, Gao QY, Fang JY. Loss of PTEN expression as a predictor of resistance to anti-EGFR monoclonal therapy in metastatic colorectal cancer: evidence from retrospective studies. Cancer Chemother Pharmacol. 2012;69: 1647-1655. doi 10.1007/s00280-012-1886-y

87. Nassif NT, Lobo GP, Wu X, et al. PTEN mutations are common in sporadic microsatellite stable colorectal cancer. Oncogene. 2004;23:617-628. doi 10 1038/sj.onc. 1207059

88. Sawai H, Yasuda A, Ochi N, et al. Loss of PTEN expression is associated with colorectal cancer liver metastasis and poor patient survival. BMC Gastroenterol. 2008;8:56. doi 10.1186/1471-230X-8-56

89. Lin MS, Huang JX, Chen WC, et al. Expression of PPARgamma and PTEN in human colorectal cancer: an immunohistochemical study using tissue microarray methodology. Oncol Lett. 2011;2:1219-1224.

90. Li XH, Zheng HC, Takahashi $\mathrm{H}$, et al. PTEN expression and mutation in colorectal carcinomas. Oncol Rep. 2009;22:757-764.

91. Colakoglu T, Yildirim S, Kayaselcuk F, et al. Clinicopathological significance of PTEN loss and the phosphoinositide 3-kinase/Akt pathway in sporadic colorectal neoplasms: is PTEN loss predictor of local recurrence? Am J Surg. 2008;195:719-725. doi 10.1016/j.amjsurg.2007.05.061

92. Eklof V, Wikberg ML, Edin S, et al. The prognostic role of KRAS, BRAF, PIK3CA and PTEN in colorectal cancer. Br J Cancer. 2013;108:2153-2163. do 10.1038/bjc.2013.212

93. Price TJ, Hardingham JE, Lee CK, et al. Prognostic impact and the relevance of PTEN copy number alterations in patients with advanced colorectal cancer (CRC) receiving bevacizumab. Cancer Med. 2013;2:277-285. doi 10. 1002/cam4.75

94. Frattini M, Saletti P, Romagnani E, et al. PTEN loss of expression predicts cetuximab efficacy in metastatic colorectal cancer patients. Br J Cancer. 2007;97: 1139-1145. doi 10.1038/sj.bjc.6604009

95. Perrone $\mathrm{F}$, Lampis $\mathrm{A}$, Orsenigo $\mathrm{M}$, et al. PI3KCA/PTEN deregulation contributes to impaired responses to cetuximab in metastatic colorectal cancer patients. Ann Oncol. 2009;20:84-90. doi 10.1093/annonc/mdn541

96. Sartore-Bianchi A, Martini M, Molinari F, et al. PIK3CA mutations in colorectal cancer are associated with clinical resistance to EGFR-targeted monoclonal antibodies. Cancer Res. 2009;69:1851-1857. doi 10.1158/00085472.CAN-08-2466

97. Negri FV, Bozzetti C, Lagrasta CA, et al. PTEN status in advanced colorectal cancer treated with cetuximab. Br J Cancer. 2010;102:162-164. doi 10.1038/sj.bjc.6605471

98. Laurent-Puig P, Cayre A, Manceau G, et al. Analysis of PTEN, BRAF, and EGFR status in determining benefit from cetuximab therapy in wild-type KRAS metastatic colon cancer. J Clin Oncol. 2009;27:5924-5930. doi 10.1200/JCO. 2008.21.6796

99. Tol J, Dijkstra JR, Klomp M, et al. Markers for EGFR pathway activation as predictor of outcome in metastatic colorectal cancer patients treated with or without cetuximab. Eur J Cancer. 2010;46:1997-2009. doi 10.1016/j.ejca.2010.03.036

100. Ulivi P, Capelli L, Valgiusti $M$, et al. Predictive role of multiple gene alterations in response to cetuximab in metastatic colorectal cancer: a single center study. J Transl Med. 2012;10:87 doi 10.1186/1479-5876-10-87

101. Cejas P, Lopez-Gomez M, Aguayo C, et al. Analysis of the concordance in the EGFR pathway status between primary tumors and related metastases of colorectal cancer patients: implications for cancer therapy. Curr Cancer Drug Targets. 2012;12:124-131. doi 10.2174/156800912799095162

102. Vakiani $E$, Janakiraman $M$, Shen $R$, et al. Comparative genomic analysis of primary versus metastatic colorectal carcinomas. J Clin Oncol. 2012;30:29562962. doi 10.1200/JCO.2011.38.2994

103. Lee KH, Kim JS, Lee CS, et al. KRAS discordance between primary and recurrent tumors after radical resection of colorectal cancers. J Surg Oncol. 2015; 111:1059-1064. doi 10.1002/jso.23936

104. Aisner DL, Deshpande C, Baloch Z, et al. Evaluation of EGFR mutation status in cytology specimens: an institutional experience. Diagn Cytopathol. 2013;41:316-323. doi 10.1002/dc.21851

105. Lindeman NI, Cagle PT, Beasley MB, et al. Molecular testing guideline for selection of lung cancer patients for EGFR and ALK tyrosine kinase inhibitors: guideline from the College of American Pathologists, International Association for the Study of Lung Cancer, and Association for Molecular Pathology. Arch Pathol Lab Med. 2013;137:828-860. doi 10.5858/arpa.20120720-OA

106. Bettegowda C, Sausen M, Leary RJ, et al. Detection of circulating tumor DNA in early- and late-stage human malignancies. Sci Transl Med. 2014;6: $224 \mathrm{ra} 224$

107. Clinical Laboratory Improvement Amendments of 1988. 42 CFR $\S 493.1351-1495$ (1992)

108. Sakai K, Tsurutani J, Yamanaka T, et al. Extended RAS and BRAF mutation analysis using next-generation sequencing. PLoS One. 2015;10:e0121891. doi 10.1371/journal.pone.0121891

109. Salto-Tellez M, Gonzalez de Castro D. Next-generation sequencing: a change of paradigm in molecular diagnostic validation. J Pathol. 2014;234:5-10. doi 10.1002/path.4365

Arch Pathol Lab Med-Vol 141, May 2017
110. Cottrell CE, Al-Kateb H, Bredemeyer AJ, et al. Validation of a nextgeneration sequencing assay for clinical molecular oncology. J Mol Diagn. 2014; 16:89-105. doi 10.1016/j.jmoldx.2013.10.002

111. Clinical laboratory improvement amendments of 1988. 42 CFR $\$ 493.1253$ (2003)

112. College of American Pathologists. CAP laboratory accreditation checklists. http://www.cap.org/apps/cap.portal. Accessed November 18, 2016

113. Clinical and Laboratory Standards Institute. Establishing molecular testing in clinical laboratory environments, approved guideline (MM19-A). Wayne, PA: Clinical Laboratory Standards Institute; 2011.

114. Halling KC, Schrijver I, Persons DL. Test verification and validation for molecular diagnostic assays. Arch Pathol Lab Med. 2012;136:11-13. doi 10. 5858/arpa.2011-0212-ED

115. Clinical and Laboratory Standards Institute. Molecular Diagnostic Methods for Solid Tumors (Nonhematological Neoplasms). (MM23-ED 1). Wayne, PA: Clinical Laboratory Standards Institute; 2015.

116. Ogino S, Gulley ML, den Dunnen JT, et al. Standard mutation nomenclature in molecular diagnostics: practical and educational challenges. J Mol Diagn. 2007;9:1-6. doi 10.2353/jmoldx.2007.060081

117. Fishel R, Lescoe MK, Rao MR, et al. The human mutator gene homolog $\mathrm{MSH} 2$ and its association with hereditary nonpolyposis colon cancer. Cell. 1993; 75:1027-1038.

118. Bronner CE, Baker SM, Morrison PT, et al. Mutation in the DNA mismatch repair gene homologue hMLH1 is associated with hereditary non-polyposis colon cancer. Nature. 1994;368:258-261. doi 10.1038/368258a0

119. Liu B, Nicolaides NC, Markowitz S, et al. Mismatch repair gene defects in sporadic colorectal cancers with microsatellite instability. Nat Genet. 1995;9:4855. doi 10.1038/ng0195-48

120. Gologan A, Sepulveda AR. Microsatellite instability and DNA mismatch repair deficiency testing in hereditary and sporadic gastrointestinal cancers. Clin Lab Med. 2005;25:179-196. [doi 10.1016/j.cll.2004.12.001

121. Fitzgibbons PL, Bradley LA, Fatheree LA, et al. Principles of analytic validation of immunohistochemical assays: guideline from the College of American Pathologists Pathology and Laboratory Quality Center. Arch Pathol Lab Med. 2014;138:1432-1443. doi 10.5858/arpa.2013-0610-CP

122. Sepulveda AR. The importance of microsatellite instability in colonic neoplasms. Medscape. 2008:571610. www.medscape.org/viewarticle/571610. Accessed November 18, 2016.

123. Hatch SB, Lightfoot HM Jr, Garwacki CP, et al. Microsatellite instability testing in colorectal carcinoma: choice of markers affects sensitivity of detection of mismatch repair-deficient tumors. Clin Cancer Res. 2005;11:2180-2187. doi 10.1158/1078-0432.CCR-04-0234

124. Watson N, Grieu F, Morris M, et al. Heterogeneous staining for mismatch repair proteins during population-based prescreening for hereditary nonpolyposis colorectal cancer. J Mol Diagn. 2007;9:472-478. doi 10.2353/jmoldx.2007. 060162

125. Cicek MS, Lindor NM, Gallinger S, et al. Quality assessment and correlation of microsatellite instability and immunohistochemical markers among populationand clinic-based colorectal tumors results from the Colon Cancer Family Registry. J Mol Diagn. 2011;13:271-281. doi 10.1016/j.jmoldx.2010.12.004

126. Wong NA, Gonzalez D, Salto-Tellez M, et al. RAS testing of colorectal carcinoma-a guidance document from the Association of Clinical Pathologists Molecular Pathology and Diagnostics Group. J Clin Pathol. 2014;67:751-757. doi 10.1136/jclinpath-2014-202467

127. Biagi JJ, Raphael MJ, Mackillop WJ, et al. Association between time to initiation of adjuvant chemotherapy and survival in colorectal cancer: a systematic review and meta-analysis. JAMA. 2011;305:2335-2342. doi 10. 1001/jama.2011.749

128. Franklin WA, Haney J, Sugita $M$, et al. KRAS mutation: comparison of testing methods and tissue sampling techniques in colon cancer. J Mol Diagn. 2010;12:43-50. doi 10.2353/jmoldx.2010.080131

129. Laosinchai-Wolf W, Ye F, Tran V, et al. Sensitive multiplex detection of KRAS codons 12 and 13 mutations in paraffin-embedded tissue specimens. J Clin Pathol. 2011;64:30-36. doi 10.1136/jcp.2010.081539

130. Ma ES, Wong CL, Law FB, et al. Detection of KRAS mutations in colorectal cancer by high-resolution melting analysis. J Clin Pathol. 2009;62: 886-891. doi 10.1136/jcp.2008.063677

131. Pinto P, Rocha P, Veiga I, et al. Comparison of methodologies for KRAS mutation detection in metastatic colorectal cancer. Cancer Genet. 2011;204: 439-446. doi 10.1016/j.cancergen.2011.07.003

132. Tol J, Dijkstra JR, Vink-Borger ME, et al. High sensitivity of both sequencing and real-time PCR analysis of KRAS mutations in colorectal cancer tissue. J Cell Mol Med. 2010;14:2122-2131. doi 10.1111/j.1582-4934.2009.00788.x

133. Buxhofer-Ausch V, Ausch C, Zeillinger R, et al. Duplex reversehybridization assay for the simultaneous detection of KRAS/BRAF mutations in FFPE-extracted genomic DNA from colorectal cancer specimens. Dis Markers. 2013;34:171-177. doi 10.1155/2013/740659

134. Carotenuto P, Roma C, Rachiglio AM, et al. Detection of KRAS mutations in colorectal carcinoma patients with an integrated PCR/sequencing and real-time PCR approach. Pharmacogenomics. 2010;11:1169-1179. doi 10.2217/pgs.10.86

135. Cavallini A, Valentini AM, Lippolis C, et al. KRAS genotyping as biomarker in colorectal cancer: a comparison of three commercial kits on histologic material. Anticancer Res. 2010;30:5251-5256.

136. Chang YS, Yeh KT, Hsu NC, et al. Detection of $\mathrm{N}_{-}, \mathrm{H}$-, and KRAS codons 12,13 , and 61 mutations with universal RAS primer multiplex PCR and N-, H-, 
and KRAS-specific primer extension. Clin Biochem. 2010;43:296-301. doi 10. 1016/j.clinbiochem.2009.10.007

137. Chen YL, Chang YS, Chang JG, et al. Genotyping of K-ras codons 12 and 13 mutations in colorectal cancer by capillary electrophoresis. J Chromatogr A. 2009;1216:5147-5154. doi 10.1016/j.chroma.2009.04.083

138. Chow L, Lin PC, Chang JS, et al. Differences in the frequencies of K-ras c12-13 genotypes by gender and pathologic phenotypes in colorectal tumors measured using the allele discrimination method. Environ Mol Mutagen. 2012; 53:22-31. doi 10.1002/em.20673

139. Kristensen LS, Daugaard IL, Christensen M, et al. Increased sensitivity of KRAS mutation detection by high-resolution melting analysis of COLD-PCR products. Hum Mutat. 2010;31:1366-1373. doi 10.1002/humu.21358

140. Kristensen LS, Kjeldsen TE, Hager $\mathrm{H}$, et al. Competitive amplification of differentially melting amplicons (CADMA) improves KRAS hotspot mutation testing in colorectal cancer. BMC Cancer. 2012;12:548. doi 10.1186/1471-2407-12-548

141. Lang AH, Drexel $H$, Geller-Rhomberg S, et al. Optimized allele-specific real-time PCR assays for the detection of common mutations in KRAS and BRAF. J Mol Diagn. 2011;13:23-28. doi 10.1016/j.jmoldx.2010.11.007

142. Sundstrom $M$, Edlund $K$, Lindell $M$, et al. KRAS analysis in colorectal carcinoma: analytical aspects of pyrosequencing and allele-specific PCR in clinical practice. BMC Cancer. 2010;10:660. doi 10.1186/1471-2407-10-660

143. Viray $\mathrm{H}$, Li K, Long TA, et al. A prospective, multi-institutional diagnostic trial to determine pathologist accuracy in estimation of percentage of malignant cells. Arch Pathol Lab Med. 2013;137:1545-1549. doi 10.5858/arpa.2012-0561-CP

144. Nardon E, Glavac D, Benhattar J, et al. A multicenter study to validate the reproducibility of $\mathrm{MSI}$ testing with a panel of 5 quasimonomorphic mononucle- otide repeats. Diagn Mol Pathol. 2010;19:236-242. doi 10.1097/PDM. 0b013e3181db67af

145. Ribic CM, Sargent DJ, Moore MJ, et al. Tumor microsatellite-instability status as a predictor of benefit from fluorouracil-based adjuvant chemotherapy for colon cancer. N Engl J Med. 2003;349:247-257. doi 10.1056/NEJMoa022289

146. Jover $R$, Zapater $P$, Castells $A$, et al. The efficacy of adjuvant chemotherapy with 5-fluorouracil in colorectal cancer depends on the mismatch repair status. Eur J Cancer. 2009;45:365-373. doi 10.1016/j.ejca.2008.07.016

147. Human Genome Variation Society. Human Genome Variation Society website. www.hgvs.org. Updated September 25, 2016. Accessed November 18, 2016.

148. Richards S, Aziz N, Bale S, et al. Standards and guidelines for the interpretation of sequence variants: a joint consensus recommendation of the American College of Medical Genetics and Genomics and the Association for Molecular Pathology. Genet Med. 2015;17:405-424. doi 10.1038/gim.2015.30

149. Sukhai MA, Craddock KJ, Thomas M et al. A classification system for clinical relevance of somatic variants identified in molecular profiling of cancer. Genet Med. 2016;18:128-136. doi 10.1038/gim.2015.47

150. Kalman LV, Lubin IM, Barker S, et al. Current landscape and new paradigms of proficiency testing and external quality assessment for molecular genetics. Arch Pathol Lab Med. 2013;137:983-988. doi 10.5858/arpa.2012-0311-RA

151. Schrijver I, Aziz N, Jennings LJ, et al. Methods-based proficiency testing in molecular genetic pathology. J Mol Diagn. 2014;16:283-287. doi 10.1016/j. jmoldx.2014.02.002

152. Diaz LA, Jr, Bardelli A. Liquid biopsies: genotyping circulating tumor DNA. J Clin Oncol. 2014;32:579-586. doi 10.1200/JCO.2012.45.2011

\begin{tabular}{|c|c|c|}
\hline \multicolumn{3}{|c|}{ APPENDIX 1. Disclosed Interests and Activities 2010 - June 2016} \\
\hline Name & Interest/Activity Type & Entity \\
\hline Federico A. Monzon, MD & Employment, Stock Options/Bonds & $\begin{array}{l}\text { InVitae, 2011-2015 } \\
\text { Castle Biosciences, 2015-present }\end{array}$ \\
\hline Antonia R. Sepulveda, MD, PhD & Consultant & $\begin{array}{l}\text { American Gastroenterological Society on } \\
\text { endoscopy and tissue sampling, } 2015\end{array}$ \\
\hline Veena M. Singh, MD & $\begin{array}{l}\text { Employment, Stock Options/Bonds } \\
\text { Consultant }\end{array}$ & $\begin{array}{l}\text { Biocept } \\
\text { bioTheranostics, Inc } \\
\text { Lab PMM }\end{array}$ \\
\hline
\end{tabular}

\section{APPENDIX 2. Disclosed Positions of Interest from 2010 - June 2016}

Carmen J. Allegra, MD

Allison M. Cushman-Vokoun, MD, PhD

William K. Funkhouser, MD, PhD

Wayne Grody, MD, PhD

Stanley R. Hamilton, MD
NRG Oncology, deputy director and GI Committee co-chair, 2014 - Present

NSABP Foundation, senior vice-president, 2014 - Present

Journal of the National Cancer Institute, editor-in-chief, 2012 - Present

CAP Molecular Diagnostic, Genomic and Precision Medicine Resource Guide, CAP PHC

Committee

Molecular Pathology Representative, ASCP Curriculum Committee

Genetics Representative, Association for Molecular Pathology (AMP) Training and Education

Committee

CAP Personalized Health Care (PHC) Committee

Molecular Oncology Committee, College of American Pathologists, 2012-2016

Association of Directors of Anatomic and Surgical Pathology (ADASP)

President, American College of Medical Genetics and Genomics, 2011-2013

$\mathrm{NCl}$ Colon Task Force, member, 2015-present

Journal of Pathology, Archives of Pathology \& Laboratory Medicine, 2015-present

$\mathrm{NCl}$ Program for Assessment of Clinical Cancer Tests (PACCT) Working Group, 2015-present

FDA Immunology Devices Panel, member, 2015-present

Actionable Genome Consortium, member, 2015-present

MolDX Advisory Panel member, Palmetto Medicare Administrative Contractor (MAC), 2015-present

College of American Pathologists co-chair of Colorectal Molecular Markers Expert Panel, Physician Quality Reporting Committee and Economic Affairs Committee, member, 2015-present

College of American Pathologists Cancer Biomarker Reporting Committee, member, 2015-present

Institute of Medicine Committee on Policy Issues in the Clinical Development and Use of Biomarkers for Molecularly Targeted Therapies, sponsor and testimony on January 29, 2015, 2015-present

Medical Evidence Development Consortium (MED-C), member, 2015-present

Center for Medicare and Medicaid Services (CMS) Medicare Evidence Development and

Coverage Advisory Committee (MEDCAC), member, 2015-present

CMS Advisory Panel on Clinical Diagnostic Laboratory Tests (CDLT), member, 2015-present 


\section{APPENDIX 2. Continued}

Scott Kopetz, MD, PhD NCl Colon Task Force, Translational Sciences Representative, 2012-2016

$\mathrm{NCl}$ Colon Task Force, Genomics Subcommittee, 2012-2016

Southwest Oncology Group, 2012-2016

Colon Cancer, National Surgical Adjuvant Breast and Bowel Project (NSABP), the Radiation Therapy Oncology Group (RTOG), and the Gynecologic Oncology Group (GOG) (NRG) Cooperative Group, 2013-2016

Gastrointestinal Steering Committee, National Cancer Institute, 2014-2016

Southwest Oncology Group (SWOG), Translational Sciences Gl Sub-Committee, 2014-2016

GI Program, NIH Cancer Center Support Grant (CCSG), 2015-2016

Christopher Lieu, MD Southwest Oncology Group (SWOG), Translational Sciences GI Sub-Committee, 2015-2016

Noralane M. Lindor, MD Genetic Subcommittee of the Cancer Prevention Committee, American Society of Clinical Oncology, 2012-2015

Federico A. Monzon, MD Association for Molecular Pathology, president, 2017

Association for Molecular Pathology, president-elect, 2016

Association for Molecular Pathology, chair, Council of MGP Directors, 2014

CAP-ASCP-AMP Guideline Development for Colorectal Cancer Molecular Testing, AMP Co-chair, 2011-2013

Association for Molecular Pathology, chair, Solid Tumor Subdivision and Council member, 2012

College of American Pathologists, Technology Assessment Committee (TAC) member, 2008-2012

Bruce D. Minsky, MD Chair of the Board, American Society for Radiation Oncology

GI Steering Committee, National Cancer Institute

Jan A. Nowak, MD, PhD CAP Center Committee, Pathology and Laboratory Quality Center, 2009 - 2015

CAP Public Health Policy Committee (formerly Patient Safety and Performance Measures Committee), 2007- 2011

CAP CGPA Molecular Pathology Working Group, 2008- 2012

Ad Hoc Committee on Laboratory Quality and Improvement for the 21st Century LQI-21 Laboratory Developed Test (LDT) Working Group, 2008 - 2010

CAP Molecular Pathology (Molecular Oncology) Resource Committee, 2005-2011

Measures and Performance Assessment Working Group, College of American Pathologists Economic Affairs Committee, 2012 - 2013

Council on Governmental and Professional Affairs - PHC Working Group, 2012 - 2016

College of American Pathologists (CAP) Personalized Healthcare Committee (PHC)

Archives of Pathology \& Laboratory Medicine, associate editor for Clinical Pathology, 2012 - current

CAP Guideline Metrics Expert Panel, member, 2014 - current

AMA CPT Editorial Panel Member (American Hospital Association), 2015 - current

AMA CPT Molecular Pathology Advisory Group (MPAG), 2015 - current

AMP Professional Relations Committee, 2006- 2012

AMP Economic Affairs Committee, 2009 - current; (co-chair, 2013 - 2014)

AMA CPT Editorial Panel Molecular Pathology Coding Working Tier 1 and Tier 2 Working Groups, 2009 2012

AMP past president, 2010

AMP Nominating Committee (chair), 2010

Pathology Coding Caucus - AMP Representative, 2005-2008; 2013 - 2015

Daniel J. Sargent, PhD

CMS Medicare Evidence Development and Advisory Committee (MEDCAC) appointee, 2012

Alliance for Clinical Trials in Oncology, 2011-present

Expert panel members and staff Joseph Willis, MD Jennifer Clark, SCT(ASCP)CM, MBCM, Carol Colasacco, MLIS, R. Bryan Rumble, MSc, Robyn Temple-Smolkin, PhD, HCLD, and Christina Ventura, MT(ASCP) have no reported conflicts of interest to disclose.

The information above reflects disclosures that were collected and reviewed by the College of American Pathologists, the American Society for Clinical Pathology, the American Society of Clinical Oncology, and the Association for Molecular Pathology. The disclosures that appear in the individual journals of the societies may vary based on journal-specific policies and procedures. 